ACT-03-03

FTUV-030826

KCL-PH/TH 030826

MIFP-03-19

UA/NPPS 26-08-03

\title{
WMAPing the Universe: Supersymmetry, Dark Matter, Dark Energy, Proton Decay and Collider Physics
}

\author{
A.B. Lahanas ${ }^{a}$, N.E. Mavromatos ${ }^{b, c}$ and D.V. Nanopoulos ${ }^{d, e, f}$ \\ ${ }^{a}$ University of Athens, Physics Department, Nuclear and Particle Physics section, \\ Panepistimioupolis, Zografou Campus, Athens GR-15771, Greece. \\ ${ }^{b}$ Kings College London, University of London, Department of Physics, Strand WC2R \\ 2LS, London, U.K. \\ ${ }^{c}$ Departamento de Física Téorica, Universidad de Valencia, E-46100, Burjassot, \\ Valencia, Spain. \\ ${ }^{d}$ George P. and Cynthia W. Mitchell Institute of fundamental Physics, Texas A \& $M$ \\ University, College Station, TX 7r843-4242, USA. \\ e Astroparticle Physics Group, Houston Advanced Research Center (HARC), The \\ Mitchell Campus, The Woodlands, TX 77381, USA \\ ${ }^{f}$ Chair of Theoretical Physics, Academy of Athens, Division of Natural Sciences, 28 \\ Panepistimiou Avenue, Athens GR-10679, Greece.
}

\begin{abstract}
In this review we critically discuss constraints on minimal supersymmetric models of particle physics as implied by the recent astrophysical observations of WMAP satellite experiment. Although the prospects of detecting supersymmetry increase dramatically, at least within the context of the minimal models, and $90 \%$ of the available parameter space can safely be reached by the sensitivity of future colliders, such as Tevatron, LHC and linear colliders, nevertheless we pay particular emphasis on discussing regions of the appropriate phase diagrams, which -if realized in nature- would imply that detection of supersymmetry, at least in the context of minimal models, could be out of colliders reach. We also discuss the importance of a precise determination of the radiative corrections to the muon anomalous magnetic moment, $g_{\mu}-2$, both theoretically and experimentally, which could lead to elimination of such "out of reach" regions in case of a confirmed discrepancy of $g_{\mu}-2$ from the standard model value. Finally, we briefly commend upon recent evidence, supported by observations, on a dark energy component of the Universe, of as yet unknown origin, covering $73 \%$ of its energy content. To be specific, we discuss how supergravity quintessence (relaxation) models can be made consistent with recent observations, which may lead to phenomenologically correct constrained supersymmetric models, accounting properly for this dark energy component. We also outline their unresolved problems.
\end{abstract}




\section{INTRODUCTION}

Particle Physics and Astrophysics have been separate for a number of years. Until a few years ago the accuracy with which astrophysical measurements were made was much lower than the corresponding one in particle physics experiments, thereby preventing a fruitful interaction between the two communities.

However, in the past decade we have witnessed spectacular progress in precision measurements in astrophysics as a result of significant improvements in terrestrial and extraterrestrial instrumentation. The (second phase of the) Hubble telescope opened up novel paths in our quest for understanding the Universe, by allowing observations on distant corners of the observable Universe that were not accessible before.

From the point of view of interest to particle physics, the most spectacular claims from astrophysics came five years ago from the study of distant supernovae (redshifts $z \sim 1$ ) by two independent groups [1. These observations pointed towards a current era acceleration of our Universe, something that could be explained either by a non-zero cosmological constant in a Friedman-Robertson-Walker-Einstein Universe, or in general by a non-zero dark energy component, which could even be relaxing to zero (the data are consistent with this possibility). This claim, if true, could revolutionize our understanding of the basic physics governing fundamental interactions in Nature. Indeed, only a few years ago, particle theorists were trying to identify (alas in vain!) an exact symmetry of nature that could set the cosmological constant (or more generally the vacuum energy) to zero. Now, astrophysical observations point to the contrary.

The skeptics may question the accuracy of the supernovae observations, given that neither the nuclear physics associated with their evolution, nor the physics involved in the intergalactic and interstellar matter are well understood to date so as to exclude the possibility that the observed effects on the dimering of the distant supernovae $(z \sim 1)$ as compared to the nearby ones $(z \ll 1)$ are due to conventional physics and are not related to the geometry of the Universe. However, there is additional evidence from quite different in origin astrophysical observations, those associated with the measurement of the cosmic microwave background radiation (CMB), which point towards the fact that $73 \%$ of the Universe vacuum energy consists of a dark (unknown) energy substance, in agreement with the (preliminary) supernovae observations. Moreover, recently [2] two more distant supernovae have been discovered $(z>1)$, exhibiting similar features as the previous measurements, thereby supporting the geometric interpretation on the acceleration of the Universe today, and arguing against the nuclear physics or intergalactic dust effects.

Above all, however, there are the very recent data from a new probe of Cosmic Microwave Background Radiation Anisotropy (Wilkinson Microwave Anisotropy Probe (WMAP)) 3]. In its first year of running WMAP measured CMB anisotropies to an unprecedented accuracy of billionth of a Kelvin degree, thereby correcting previous mea- 
surements by the Cosmic Background Explorer (COBE) satellite 4] by several orders of magnitude. This new satellite experiment, therefore, opened up a new era for astroparticle physics, given that such accuracies allow for a determination (using best fit models of the Universe) of cosmological parameters [5], and in particular cosmological densities, which, as we shall discuss in this review, is quite relevant for constraining models of particle physics to a significant degree.

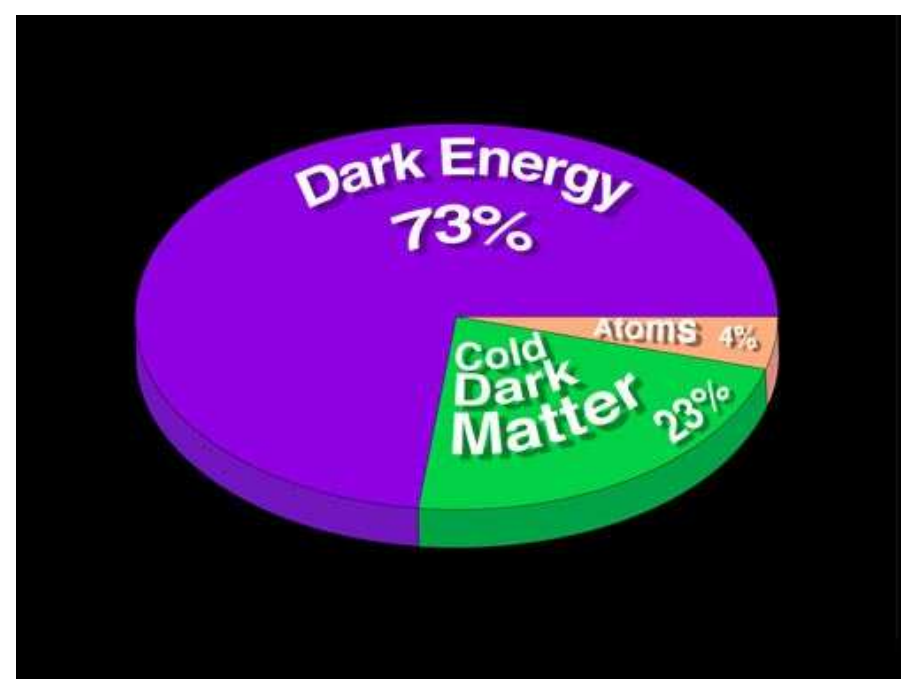

Figure 1: The energy content of our Universe as obtained by fitting data of WMAP satellite. The chart is in perfect agreement with earlier claims made by direct measurements of a current era acceleration of the Universe from distant supernovae type Ia (courtesy of http:/(map.gsfc.nasa.gov/).

The WMAP satellite experiment determined the most important cosmological parameters that could be of relevance to particle physicists, namely [5]: the Hubble constant, and thus the age of the Universe, the thickness of the last scattering surface (c.f. below), the dark energy and dark matter content of the Universe (to an unprecedented accuracy) (c.f. figure 1), confirming the earlier claims from supernovae Ia data [1, and provided evidence for early reionization $(z \sim 20)$, which, at least from the point of view of large scale structure formation, excludes Warm Dark Matter particle theory models.

In this review we shall first describe briefly the above-mentioned measurements, and then use them to constrain certain particle physics supersymmetric models (in particular, the minimally supersymmetric model, constrained by its embedding in a minimal supergravity model (mSUGRA)). We shall give a critical discussion on the derived constraints, and discuss the capability of observing Supersymmetry in colliders after these latest WMAP data. We shall pay particular attention to discussing regions of the param- 
eter space of the models, which if realized in nature, would imply impossibility of observing supersymmetry at LHC and/or linear colliders, even in the context of the mSUGRA models. In this respect we shall also discuss the importance of the $g_{\mu}-2$ experiments of the muon gyromagnetic ratio, and how certain results, pointing towards a discrepancy between the measured $g_{\mu}-2$ from the value calculated within the standard model, could eliminate the above-mentioned precarious regions of parameter space.

An important comment we would like to make in this review will concern the dark energy component $(73 \%)$ of the Universe. The WMAP measured equation of state for the Universe $p=w \rho$, with $p$ the pressure and $\rho$ the energy density, implies $-1 \leq w<-0.78$ (assuming the lower bound for theoretical reasons, otherwise the upper limit may be larger [5]). For comparison we note that $w=-1$ characterizes a perfect fluid Universe with non-zero, positive, cosmological constant. As we shall remark, supergravity quintessence models do have this feature of $w \rightarrow-1$, and it may well be that by exploiting further the data on this dark energy component of the Universe one may arrive at the physically correct supergravity model which could constrain the supersymmetric particle physics models.

The outline of this review is as follows: In section two we shall discuss briefly the WMAP experiment and its measurements, with emphasis on cosmological parameters relevant to particle physics models. In particular, we shall describe the measurements of the equation of state of quintessence models, in an attempt to shed light on the nature of the dark energy. In section three we shall discuss the basics of inflationary models, which seem to be favoured by WMAP, but we shall be critical in our discussion, stressing the current inadequacies of the observations. In section four we shall embark on the main point of our discussion, namely the connection of the WMAP measurements and Supersymmetry. Specifically, we shall discuss various models of SUSY Dark Matter, and argue that only Cold Dark Matter (CDM) is favoured by the data, given that Hot Dark Matter (neutrino) is excluded in view of the strict upper bounds of neutrino masses imposed by the WMAP, and Warm Dark Matter models with a low mass gravitino (less than $10 \mathrm{KeV}$ ) are also excluded on account of evidence for early $(z \sim 20)$ reionization of the Universe. By concentrating, for the purposes of this review, on CDM, made exclusively of neutralinos (viewed as the Lightest supersymmetric particle (LSP)) [6], we shall describe, in section five, the WMAP-derived constraints on the mSUGRA model, and the prospects for detection of supersymmetry within the context of this model in future colliders, as well as direct dark matter searches. Also we shall discuss briefly how such constraints, when combined with proton decay current lower limits, affect grand unified supersymmetric theories, specifically flipped SU(5) models. Finally, in section six we shall review critically the above constraints in light of the fact that mSUGRA and all the existing particle physics models do not take proper account of the observed dark energy component. In this respect we shall discuss supergravity/superstring/brane inspired models with relaxing to zero "vacuum energies and argue how such models may be used in the future to constrain particle 
physics supersymmetric models. The issue is of course still unresolved, as it involves the yet unsettled theoretical task of determining the precise mechanism of low-energy supersymmetry breaking. Conclusions and outlook, outlining possible future directions, will be presented in section seven.

\section{WMAP \& (ASTRO)PARTICLE PHYSICS}

\subsection{What is WMAP}

The Wilkinson Microwave Anisotropy Probe (WMAP) $[\underline{3}$ is a satellite experiment (figure 2) dedicated to measure temperature fluctuations of the cosmic microwave background (CMB), with unprecedented accuracy, as compared to previous COBE measurements, which reaches billionth of a Kelvin degree.
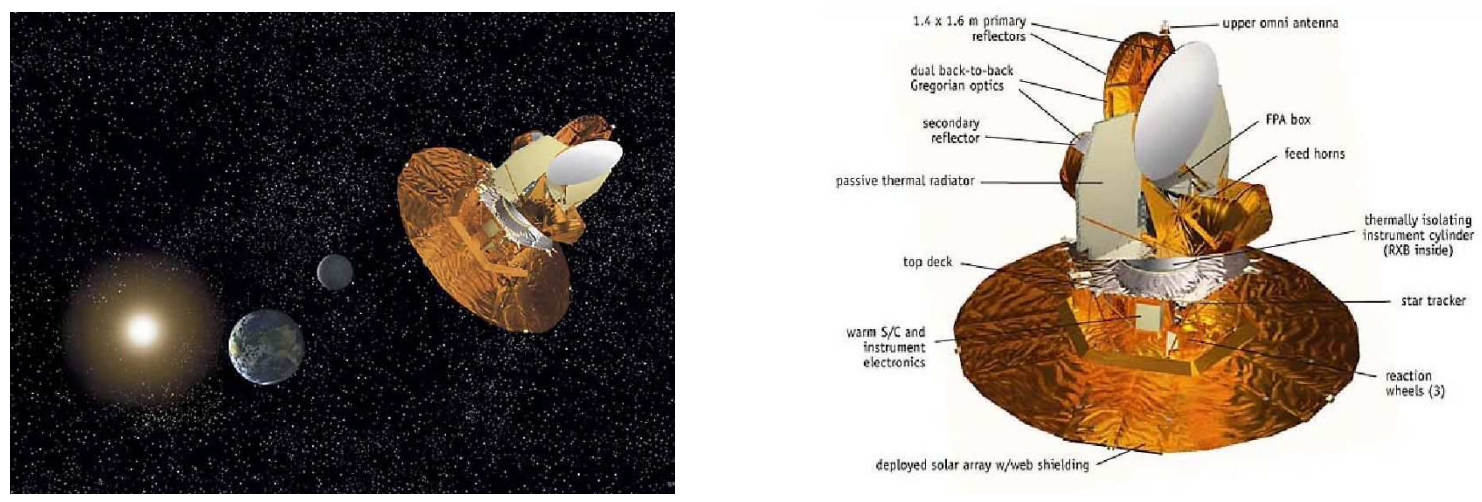

Figure 2: A graphical picture of the WMAP satellite (courtesy of http://map.gsfc.nasa.gov/).

The cosmic microwave background is the afterglow radiation left over from the hot Big Bang. Its temperature is extremely uniform all over the sky. However, tiny temperature variations or fluctuations (at the part per million level) can offer great insight into the origin, evolution, and content of the Universe. The light that is reaching us has been stretched out as the Universe has stretched, so light that was once beyond gamma rays is now reaching us in the form of microwaves (longer wavelength) (figure 3). We can only observe light that comes from the time of last scattering, in a completely analogous way as in a cloudy sky we can only see light coming from the cloud surface that is facing the earth ground (c.f. figure 4). We remind the reader that the existence of the last scattering surface is due to the fact that the Universe expands rapidly and, as a result, there is significant dilution in the density of matter particles, including radiation. Hence, 
the scattering rate of matter and also of light is diminished, and eventually stops (freezing out), implying a surface of last scattering.
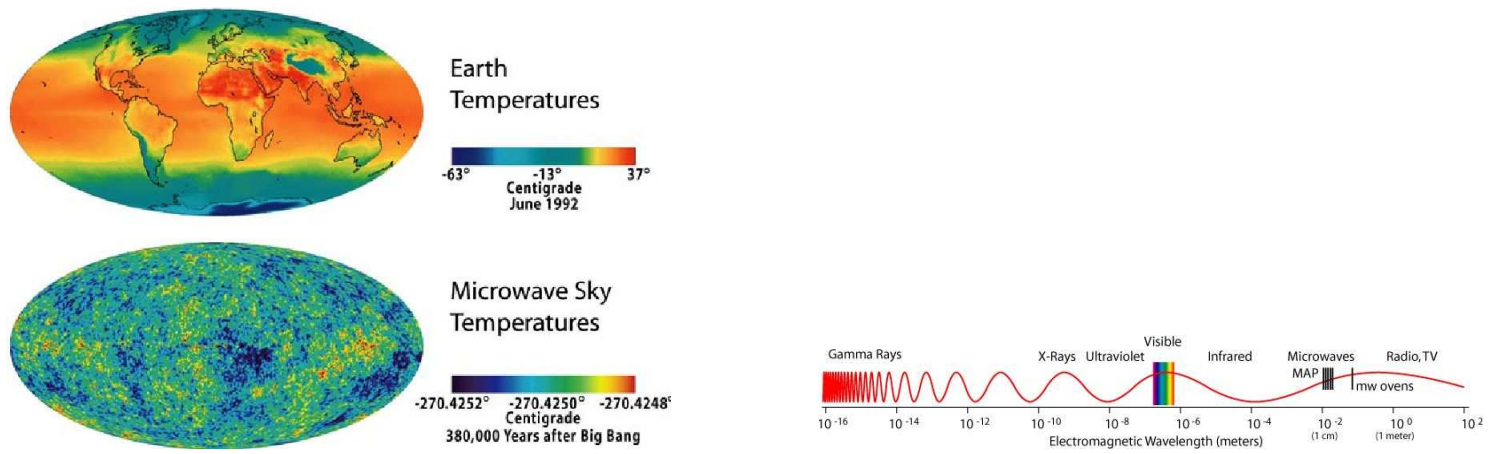

Figure 3: The expansion of the Universe causes stretching of the wavelengths of light, so light emitted at a gamma ray region of wavelengths reaches us today as microwave radiation (courtesy of http://map.gsfc.nasa.gov/).

In its first year of running, WMAP already provides a much more detailed picture of the temperature fluctuations than its COBE predecessor (figure 5), which can be analyzed to provide best fit models for cosmology, leading to severe constraints on the energy content of various model Universes, useful for particle physics, and in particular supersymmetric searches.

Theoretically [7], the temperature fluctuations in the CMB radiation are attributed to: (i) our velocity w.r.t cosmic rest frame, (ii) gravitational potential fluctuations on the last scattering surface (Sachs-Wolf effect), (iii) Radiation field fluctuations on the last scattering surface, (iv) velocity of the last scattering surface, and (v) damping of anisotropies if Universe reionizes after decoupling.

The fluctuations due to (i) imply a dipole anisotropy, while (ii)are the dominant effects for large angular scales $\theta>>1^{\circ}$. The fluctuations (iii) - (v) on the other hand, are dominant for $\theta<<1^{\circ}$. The value of $\theta=1^{o}$ is determined by the physics of decoupling.

The CMB anisotropies in the Sky are expanded as:

$$
\frac{\delta T}{T}(\theta, \phi)=\sum_{\ell=2}^{+\infty} \sum_{m=-\ell}^{+\ell} a_{\ell m} Y_{\ell m}(\theta, \phi)
$$

where $Y_{\ell m}(\theta, \phi)$ are spherical harmonics; isotropy implies $\left\langle a_{\ell m}\right\rangle=0$. Let us denote the variance of $a_{\ell m}$ by $C_{\ell}=<\left|a_{\ell m}\right|^{2}>$. A non zero $C_{\ell}$ would imply temperature fluctuations. More accurately, within a Gaussian model with width $\sigma$, the above sum over $\ell$ in the 

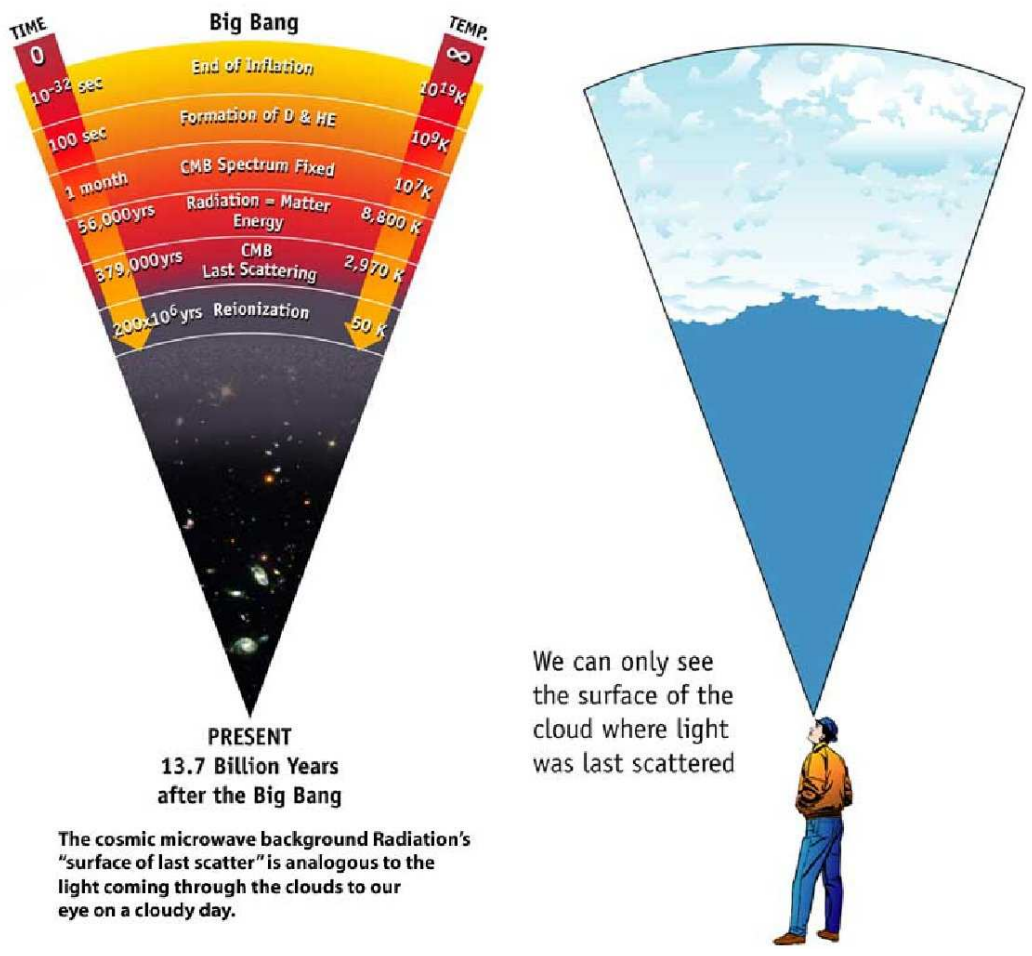

Figure 4: We can only see the light emitted from the surface where it was last scattered. This happens when we look either at a cloudy sky, or at the cosmic microwave background radiation (courtesy of http://map.gsfc.nasa.gov/).

expression for $\delta T / T$ is effectively cutoff at $\ell \sim \sigma$ :

$$
C_{\sigma}=<\frac{\delta T\left(\vec{x}_{1}\right)}{T} \frac{\delta T\left(\vec{x}_{2}\right)}{T}>=\frac{1}{4 \pi} \sum_{\ell=2}^{+\infty}(2 \ell+1)<\left|a_{\ell m}\right|^{2}>P_{\ell}\left(\vec{x}_{1}, \vec{x}_{2}\right) e^{-\left(\frac{\ell+1}{2}\right)^{2} \sigma^{2}}
$$

This Gaussian model of fluctuations is in very good agreement with the recent WMAP data (see figure 66). The perfect fit of the first few peaks to the data allows a precise determination of the total density of the Universe, which as we shall discuss next implies its spatial flatness.

\subsection{WMAP Measurement of Cosmological Parameters}

The measurements of the WMAP [5] on the cosmological parameters of interest to us here can be summarized in the figures[7] 9 see Refs. [5], with $\Omega_{i}=\rho_{i} / \rho_{c} . \rho_{i}$ is the matter-energy 

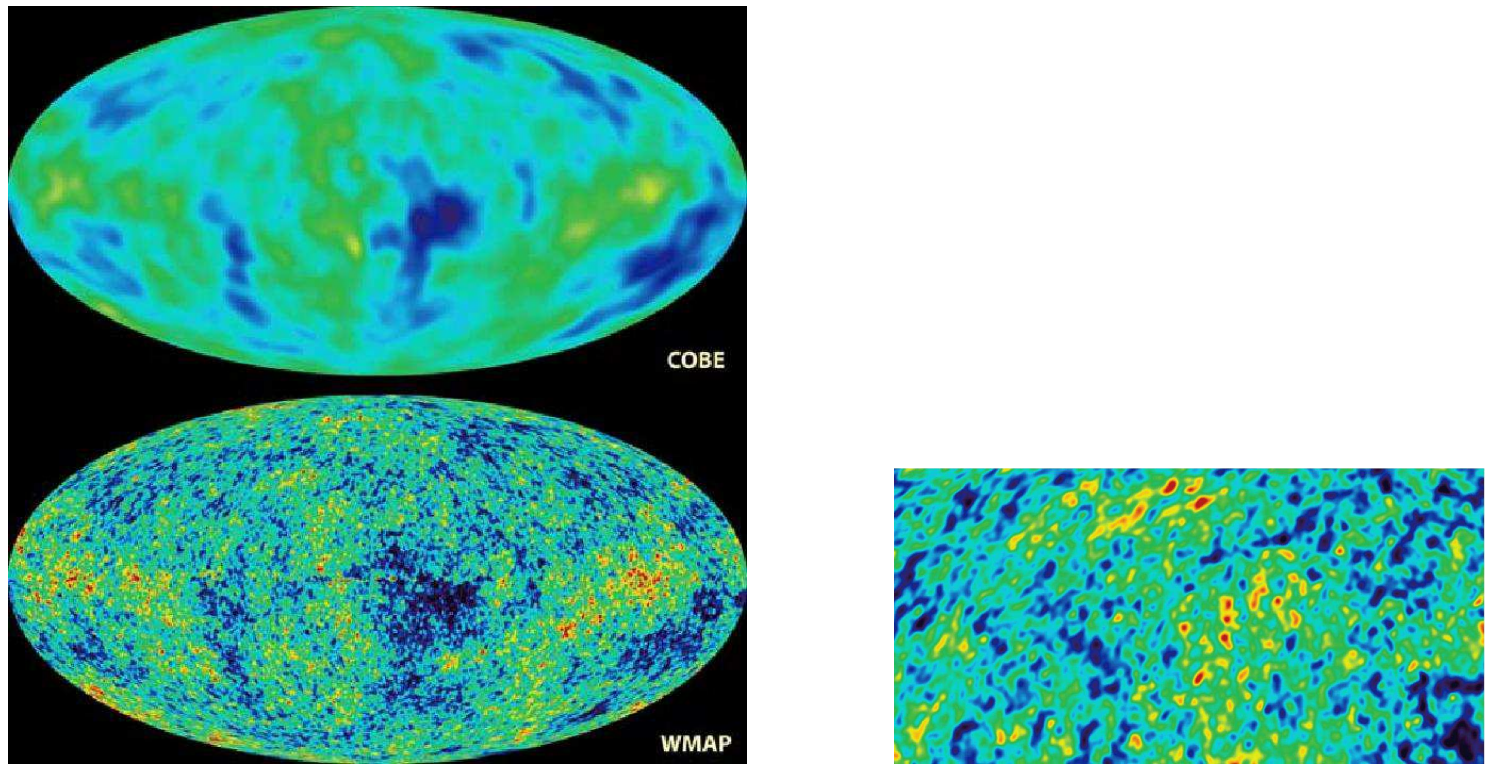

Figure 5: CMB Temperature fluctuations provided by WMAP and comparison with COBE (left), Importance of Detail (right) (courtesy of http://map.gsfc.nasa.gov/).

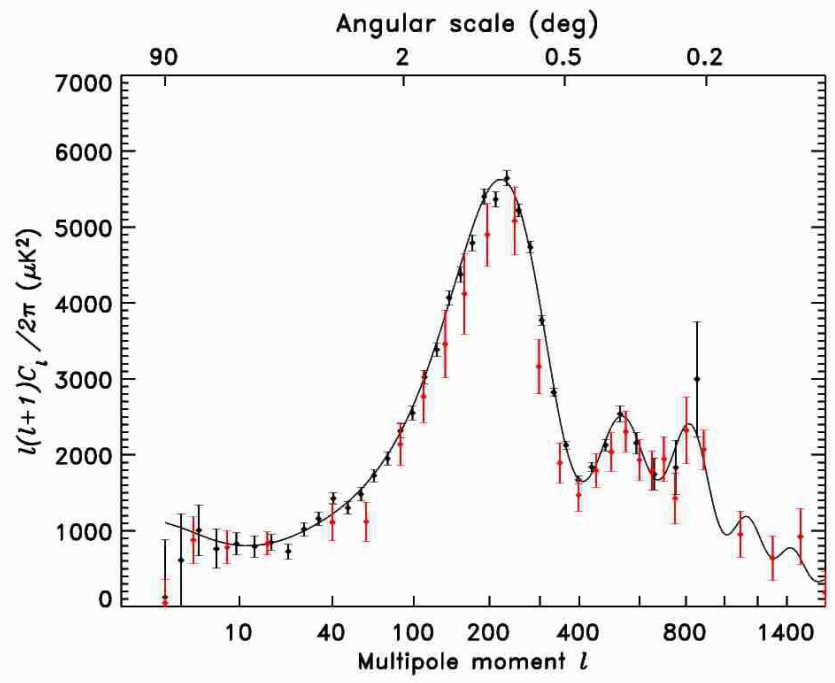

Figure 6: Red points (larger errors) are previous measurements. Black points (smaller errors) are WMAP measurements (Hinshaw, G. et al. arXiv:astro-ph/0302217). 
density component, $\rho_{c}=3 H^{2} / 8 \pi G_{N}$ (with $G_{N}$ the Newton constant) the critical density for the closure of the Universe, determined within the Friedman-Robertson-Walker framework, and $h$ the rescaled Hubble parameter, defined through $H=100 h \mathrm{Km} \mathrm{s}^{-1} \mathrm{Mpc}^{-1}$. The WMAP satellite can measure directly $\Omega h^{2}$, which is Hubble parameter independent by construction, and can also separately measure $h$. For our purposes in this article we concentrate on the measurements of the density of matter and dark energy (see figure 8), as well as on the equation of state of the dark energy component (c.f. figure 9).
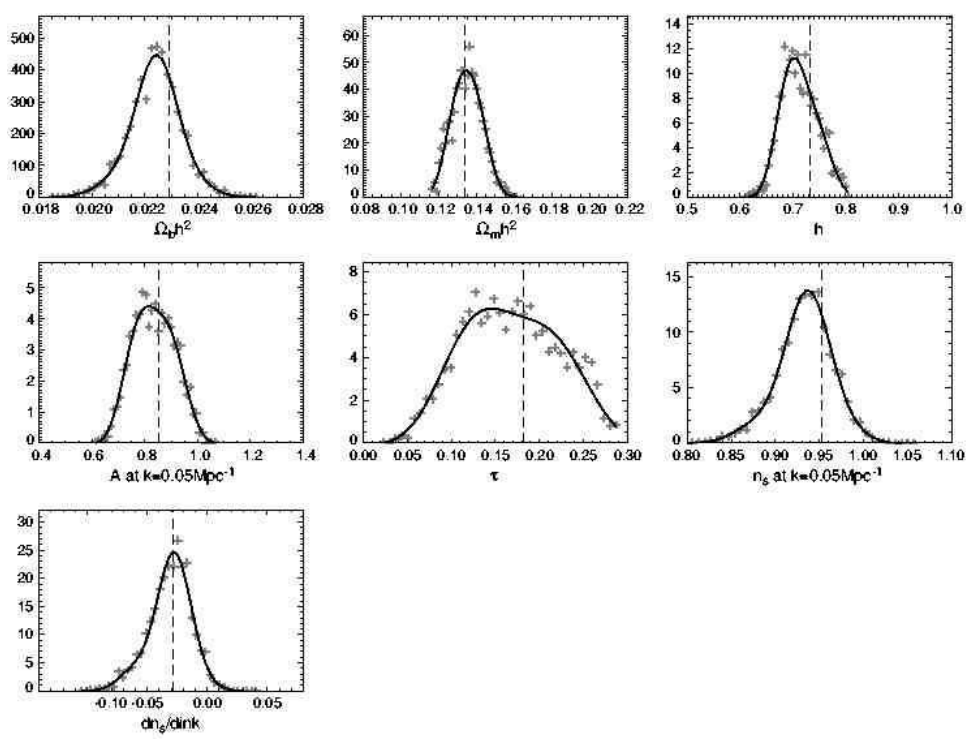

Figure 7: Likelihood Functions for various cosmological parameters measured by WMAP [5].

We observe from figure 9 that the WMAP results constrain severely the equation of state $p=w \rho$ ( $p=$ pressure), pointing towards $w<-0.78$, if one fits the data with the assumption $-1 \leq w$ (we note for comparison that in the scenarios advocating the existence of a cosmological constant one has $w=-1$ ). Many quintessence models can easily satisfy the criterion $-1<w<-0.78$, especially the supersymmetric ones, which we shall comment upon later in the article. Thus, at present, the available data are not sufficient to distinguish the cosmological constant model from quintessence (or more generally from relaxation models of the vacuum energy).

The results of the WMAP analysis (alone),including directly measurable and derived quantities, are summarized in the tables appearing in figures 1011.

One therefore obtains the chart for the energy and matter content of our Universe depicted in figure 1. This chart is in perfect agreement with direct evidence on acceleration of the Universe (and hence cosmological constant) from Supernovae Ia Data [1] (c.f. figure 

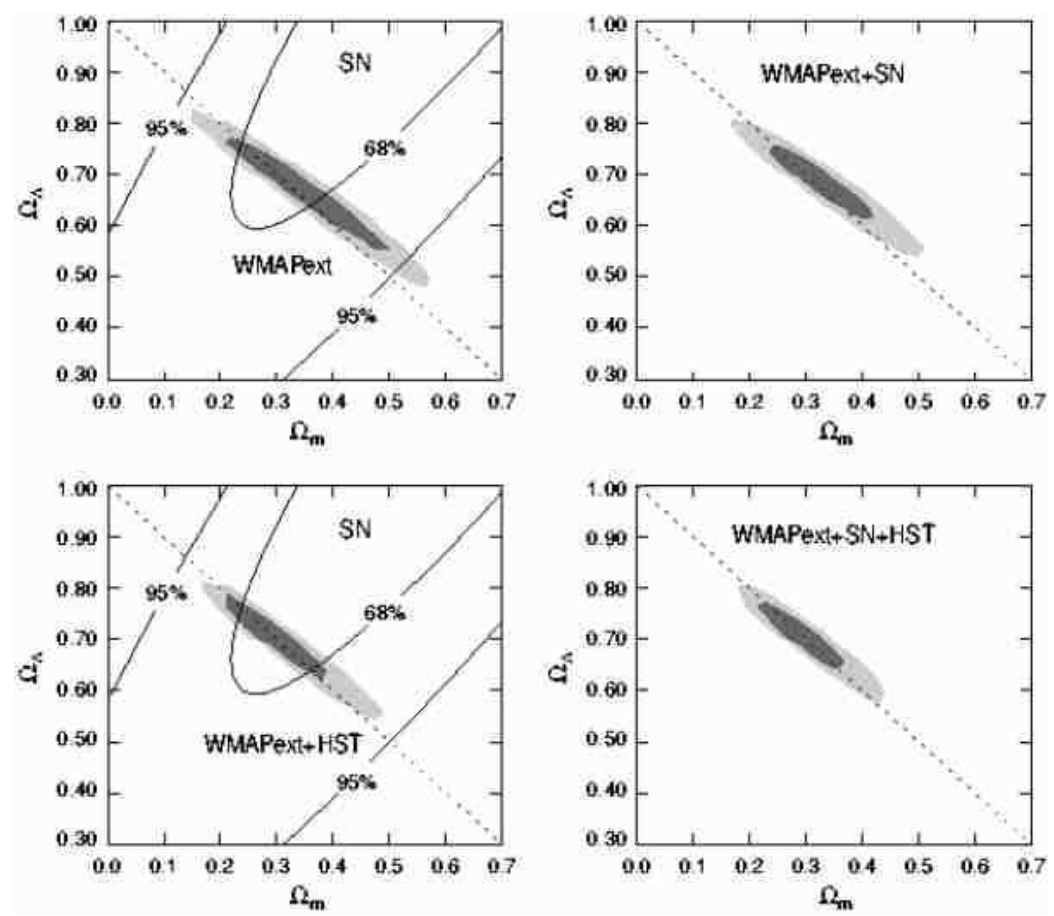

Figure 8: Energy density and matter density content of the Universe as measured by the WMAP satellite alone, as well as in combination with other experiments [5].

$12)$.

It should be stressed that the interpretation of the supernovae data is based on a best fit Friedmann-Robertson-Walker (FRW) Universe [1]:

$$
0.8 \Omega_{M}-0.6 \Omega_{\Lambda} \simeq-0.2 \pm 0.1, \quad \text { for } \Omega_{M} \leq 1.5
$$

with $\Omega_{M, \Lambda}$ corresponding to the matter and cosmological matter densities. Assuming a flat model $(\mathrm{k}=0), \Omega_{\text {total }}=1$, as supported by the CMB data, the SNIa data alone imply:

$$
\Omega_{M}^{\text {Flat }}=0.28_{-0.08}^{+0.09}(1 \sigma \text { stat. })_{-0.04}^{+0.05} \text { (identified syst.) }
$$

The deceleration parameter defined as $q \equiv-\frac{\ddot{a} a}{\dot{a}^{2}}$, where $a$ is the cosmic scale factor, receives the following form if we omit the contribution of photons which is very small,

$$
q=\frac{1}{2} \Omega_{M}-\Omega_{\Lambda} \simeq-0.57<0, \quad\left(\Omega_{\Lambda} \simeq 0.7\right) .
$$

Hence (11) and (2) provide evidence for a current era acceleration of the Universe. At this stage it should be stressed that the recent observation of two more supernovae at $z>1[2$ 

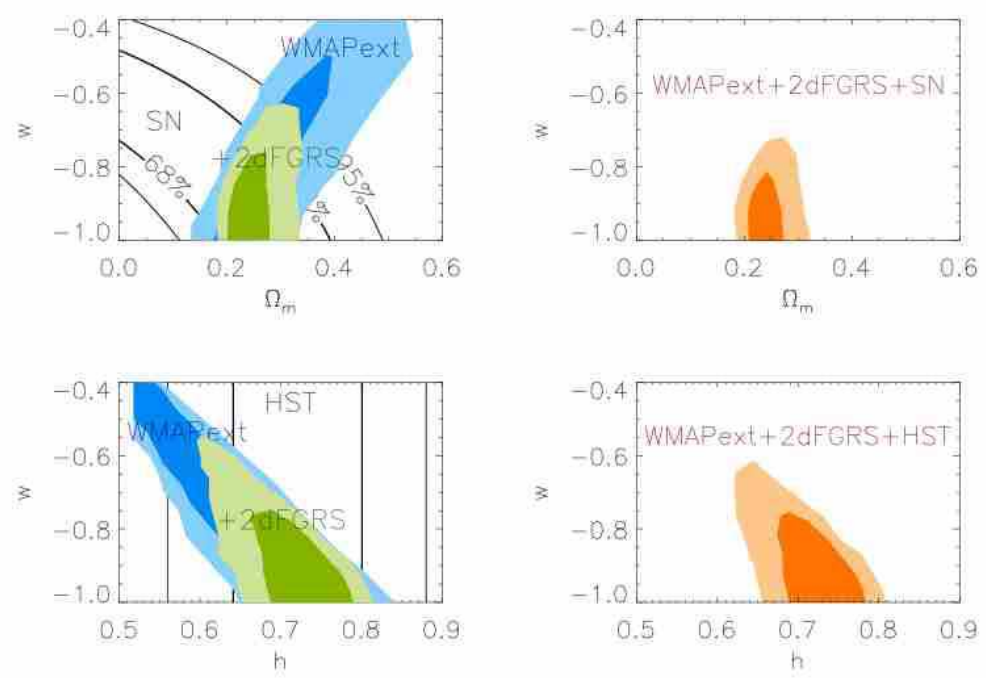

Figure 9: Equation of State of the Dark Energy component of the Universe as measured by the WMAP satellite alone, as well as in combination with other experiments [5].

supports the geometrical interpretation on the existence of a dark energy component of the Universe, and argues rather against the rôle of nuclear (evolution) or intergalactic dust effects.

The recent data of WMAP satellite lead to a new determination of $\Omega_{\text {total }}=1.02 \pm 0.02$, where $\Omega_{\text {total }}=\rho_{\text {total }} / \rho_{c}$, due to high precision measurements of secondary (two more) acoustic peaks as compared with previous CMB measurements (c.f. figure 6). Essentially the value of $\Omega$ is determined by the position of the first acoustic peak in a Gaussian model, whose reliability increases significantly by the discovery of secondary peaks and their excellent fit with the Gaussian model [5].

\section{WMAP AND INFLATION}

\subsection{Basics of Inflationary Models}

The recent determination of the cosmological parameters by the WMAP team [5] favours, by means of best fit procedure, spatially flat inflationary models of the Universe [8]. In terms of Robertson-Walker metrics

$$
d s^{2}=-d t^{2}+a^{2}(t)\left(\frac{d r^{2}}{1-k r^{2}}+r^{2} d \Omega^{2}\right)
$$


TABLE 1

Power Law ACDM Model Parameters- WMAP Data Only

\begin{tabular}{llll}
\hline \hline \multicolumn{1}{c}{ Parameter } & & Mean $(68 \%$ confidence range) & Maximum Likelihood \\
\hline Baryon Density & $\Omega_{b} h^{2}$ & $0.024 \pm 0.001$ & 0.023 \\
Matter Density & $\Omega_{m} h^{2}$ & $0.14 \pm 0.02$ & 0.15 \\
Hubble Constant & $h$ & $0.72 \pm 0.05$ & 0.68 \\
Amplitude & $A$ & $0.9 \pm 0.1$ & 0.80 \\
Optical Depth & $\tau$ & $0.166_{-0.071}^{+0.076}$ & 0.11 \\
Spectral Index & $n_{s}$ & $0.99 \pm 0.04$ & 0.97 \\
& $\chi_{\text {eff }}^{2} / \nu$ & & $1431 / 1342$ \\
\hline
\end{tabular}

${ }^{\text {a }}$ it to WMAP data only

Figure 10: Cosmological parameters measured by WMAP (only):directly measurable quantities [5].

such models are described by an early phase in which the scale factor $a(t)$ is exponentially expanding with the Robertson-Walker time $t: a(t) \sim e^{H t}$, where $H$ is the Hubble parameter. Recent WMAP data confirm its value during inflation to be of order $H=$ const $=10^{-5} M_{P l}$, where $M_{P l}$ is the Planck energy scale $\left(\sim 10^{19} \mathrm{GeV}\right)$. Spatially flat models are described by $k=0$, since the spatial curvature is proportional to this parameter, and they correspond to a total energy density $\Omega_{\text {total }}=1$.

Such a spatially flat Universe is preferred in inflationary scenaria 9]. An important feature of conventional inflationary models is the presence of a scalar mode, the inflaton $\phi$ [10, which couples to a de Sitter Universe. The field's slow rolling down the hill of its potential (c.f. figure 13) causes the Universe to expand exponentially. Its equation of motion in a de Sitter background has a frictional form, with the friction provided by the Hubble parameter $H$ (assumed almost constant during inflation):

$$
\ddot{\phi}+3 H \dot{\phi}+\frac{\delta V(\phi)}{\delta \phi}=0 \text {. }
$$

where the overdot denotes derivative w.r.t Robertson-Walker time $t$.

The quantum fluctuations of a Planck-size $\left(10^{-35}\right.$ meters $)$ Universe, which is assumed to be the state of the Universe right after the big bang in inflationary scenaria are enormously enhanced (c.f. figure 13) by the exponential expansion and result to cosmic microwave background radiation anisotropies. They may also provide the explanation of the 
TABLE 1

Derived Cosmological Parameters

\begin{tabular}{ll}
\hline \hline \multicolumn{1}{c}{ Parameter } & Mean (68\% confidence range) \\
\hline Amplitude of Galaxy Fluctuations & $\sigma_{8}=0.9 \pm 0.1$ \\
Characteristic Amplitude of Velocity Fluctuations & $\sigma_{8} \Omega_{m}^{0.6}=0.44 \pm 0.10$ \\
Baryon Density/Critical Density & $\Omega_{b}=0.047 \pm 0.006$ \\
Matter Density/Critical Density & $\Omega_{m}=0.29 \pm 0.07$ \\
Age of the Universe & $t_{0}=13.4 \pm 0.3 \mathrm{Gyr}$ \\
Redshift of Reionization (ionization fraction, $\left.x_{e}=1\right)$ & $z_{r}=17 \pm 5$ \\
Redshift at Decoupling & $z_{d e c}=1088_{-2}^{+1}$ \\
Age of the Universe at Decoupling & $t_{d e c}=372 \pm 14 \mathrm{kyr}$ \\
Thickness of Surface of Last Scatter & $\Delta z_{d e c}=194 \pm 2$ \\
Thickness of Surface of Last Scatter & $\Delta t_{d e c}=115 \pm 5 \mathrm{kyr}$ \\
Redshift at Matter/Radiation Equality & $z_{e q}=3454_{-392}^{+385}$ \\
Sound Horizon at Decoupling & $r_{s}=144 \pm 4 \mathrm{Mpc}$ \\
Angular Diameter Distance to the Decoupling Surface & $d_{A}=13.7 \pm 0.5 \mathrm{Gpc}$ \\
Acoustic Angular Scale $\left(l_{A}=\pi d_{A} / r_{s}\right)$ & $\ell_{A}=299 \pm 2$ \\
Current Density of Baryons & $n_{b}=(2.7 \pm 0.1) \times 10^{-7} \mathrm{~cm}^{-3}$ \\
Baryon/Photon Ratio & $\eta=\left(6.5_{-0.3}^{+0.4}\right) \times 10^{-10}$ \\
\hline
\end{tabular}

Figure 11: Cosmological parameters measured by WMAP (only):derived quantities [5]. 

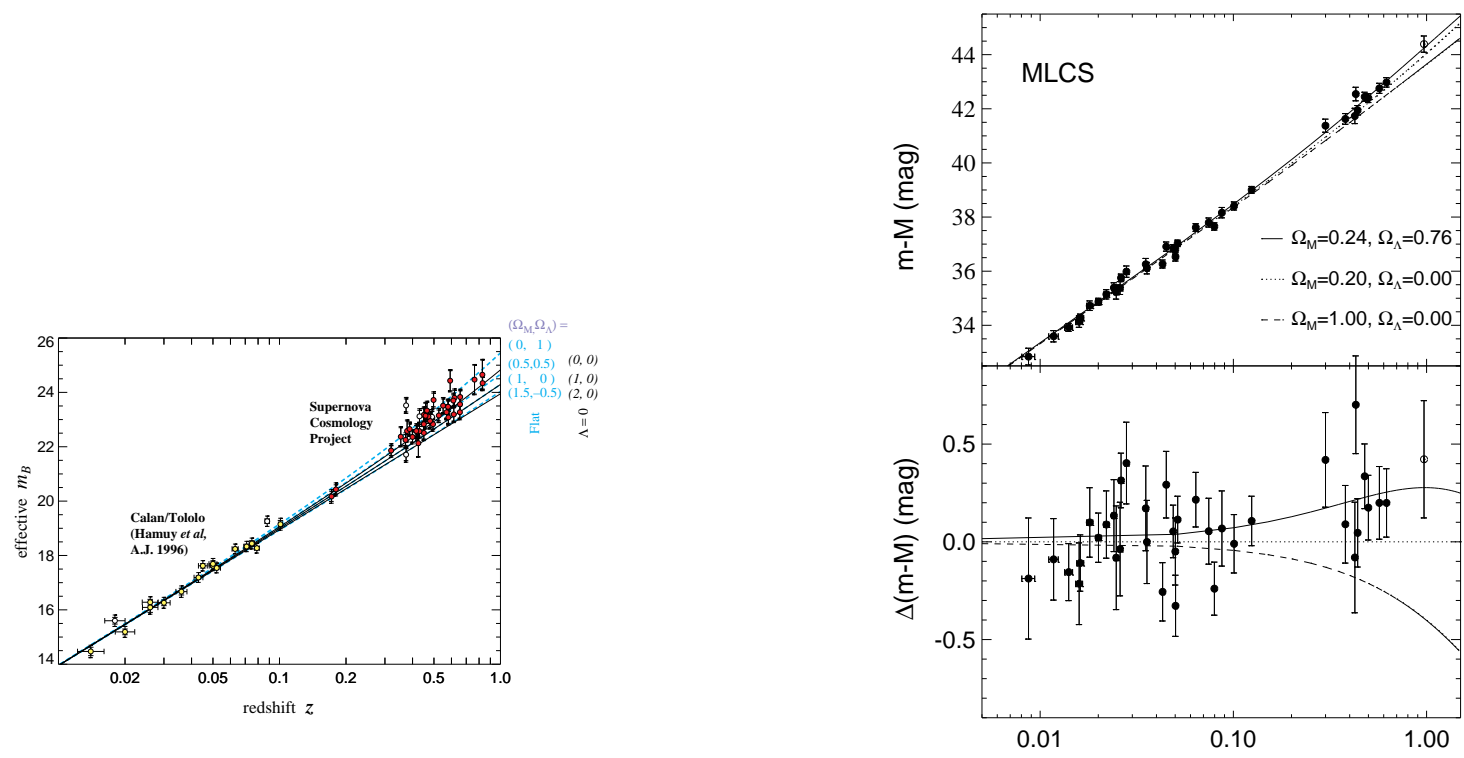

Figure 12: The only Direct evidence for acceleration of the Universe (and hence Dark energy) so far is provided by high redshift $(z \leq 1)$ supernovae measurements (SNIa) [1], which were prior to WMAP data.

presently observed large scale structure of the Universe.

The (thermal) history of the inflationary Universe [7] is quite different from the one expected in standard cosmology (c.f. figure 14). During the exponential expansion the Universe cools down significantly, and then is reheated after the end of inflation. The physics of reheating is not well understood at present, and many scenaria exist in the literature on this issue, some of which involve fundamental strings. In the present article we would not like to discuss such issues, as they lie outside our scope. An important characteristic of inflation is the enormous entropy production at the end of inflation.

\subsection{WMAP-Measured Inflationary Parameters}

The measurements of the WMAP satellite which support inflation are associated with the so called Running Spectral Index, a quantity defined as follows [11].

Let one consider density fluctuations in the Universe,

$$
\delta(\vec{x})=\frac{\rho(\vec{x})-\bar{\rho}}{\bar{\rho}}
$$

where barred quantities denote average over the Universe volume $V$. Taking the Fourier transform: $\delta(\vec{x})=\frac{V}{(2 \pi)^{3}} \int d^{3} k \delta_{\vec{k}} e^{i \vec{k} \cdot \vec{x}}$, one can define the power spectrum of the fluctuations 

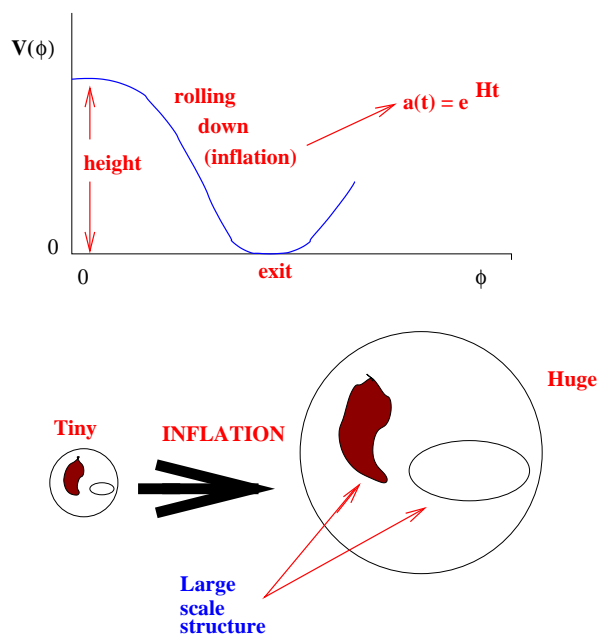

Figure 13: Inflation Basics: the inflaton and its rolling down the hill phase which causes inflation (exponential expansion); due to this expansion small quantum fluctuations may enhance and be responsible for large scale structures of the Universe at present.

as 7

$$
<\delta(\vec{x}+\vec{r}) \delta(\vec{x})>\equiv \xi=\frac{1}{(2 \pi)^{3}} V \int d^{3} k\left|\delta_{\vec{k}}\right|^{2} e^{-i \vec{k} \cdot \vec{r}}
$$

Then, in terms of a comoving scale $k$, one can define the primordial power spectrum of scalar density fluctuations [1]:

$$
P(k) \equiv\left|\delta_{\vec{k}}\right|^{2} .
$$

The running scalar spectral index $n_{s}(k)$ is

$$
n_{s}(k)=\frac{d \ln P(k)}{d \ln k} .
$$

One may fix $n_{s}$ and its slope w.r.t. $k$ at $k_{0}=0.05 M p c^{-1}$ and it is customary to assume [1] that $\frac{d^{2} n_{s}}{d \ln k^{2}}=0$, from which it follows that

$$
n_{s}(k)=n_{s}\left(k_{0}\right)+\frac{d n_{s}}{d \ln k} \ln \left(\frac{k}{k_{0}}\right) .
$$

Then the power spectrum $P(k)$ above is written as

$$
P\left(k_{0}\right)=P\left(k_{0}\right)\left(\frac{k}{k_{0}}\right)^{n_{s}\left(k_{0}\right)+\frac{1}{2} \frac{d n_{s}}{d \ln k} \ln \left(\frac{k}{k_{0}}\right)} .
$$




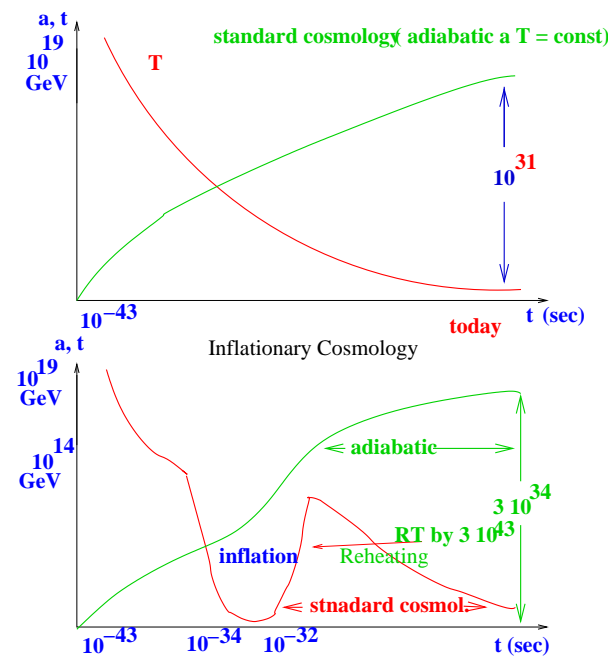

Figure 14: The thermal history of the inflationary Universe as compared to the standard cosmology: the exponential expansion causes the Universe to cool down significantly, before is reheated again. The physics of reheating is not well understood at present. The inflationary phase results in an enormous entropy production at the end of inflation.

The quantity $n_{s}(k)$ carries information on the field derivatives of the inflaton potential $V(\phi)$ in best fit models: $V^{\prime}, V^{\prime \prime}, V^{\prime \prime \prime}\left(V^{\prime} \equiv \frac{d}{d \phi} V(\phi)\right)$, and can be measured by WMAP data. In fact, standard inflation implies a constant $n_{s}$, i.e. scale invariance of the fluctuation spectrum. The current value of the running of the spectral index by the available set of WMAP data at present is very small but non-zero, however there is not enough statistics as yet to arrive at definite conclusions on the shape of the inflaton potential.

Below we give a list of the basic WMAP measurements that favour inflation [5]. For definiteness we give first the conventional slow-roll inflationary parameters [7, 9]

$$
\epsilon \equiv \frac{1}{2} M_{P}^{2}\left(\frac{V^{\prime}}{V}\right)^{2}, \eta \equiv M_{P}^{2}\left(\frac{V^{\prime \prime}}{V}\right), \xi \equiv M_{P}^{4}\left(\frac{V V^{\prime \prime \prime}}{V^{2}}\right)
$$

where $M_{P}=\left(\frac{1}{8 \pi G_{N}}\right)^{1 / 2} \simeq 2.4 \times 10^{18} \mathrm{GeV}$ is the four-dimensional Planck mass, wth $G_{N}$ the Newton constant, $V(\phi)$ is the inflaton $(\phi)$ potential, and the prime indicates derivative with respect to $\phi$. WMAP has measured the following (c.f. figs. 10 11) [3, 5]:

- Flatness of the Universe $(k=0)$

- Density fluctuations $\Delta_{R}^{2}$, which are expressed in terms of (뾰) as: $\Delta_{R}^{2}=\frac{V}{24 \pi^{2} M_{P}^{2} \epsilon}=$ $2.95 \times 10^{-9} A, \quad$ WMAP result: $A=0.77 \pm 0.07$ 
- WMAP results alone are too crude 12 to determine the shape and height of inflaton potential. When, and only when, the WMAP data are combined with all other data, then they seem to exclude, at $3-\sigma$ level, a $V \sim \phi^{4}$ potential [5].

- The ratio of tensor to scalar perturbations (at quadrupole scale) $r=\frac{A_{T}}{A_{S}}=16 \epsilon$, WMAP result $: r \simeq 0.16$

- The Spectral Index for (i) scalar density perturbations $n_{s}=1-6 \epsilon+2 \eta \quad$ WMAP result: $n_{s} \simeq 0.96$, and (ii) for tensor perturbations $n_{T}=-2 \epsilon$.

- The running of Scalar Spectral Index, a quite important WMAP result, $\frac{d n_{s}}{d \ln k}=$ $\frac{2}{3}\left[\left(n_{s}-1\right)^{2}-4 \eta^{2}\right]+2 \xi, \quad$ WMAP result: $\frac{d n_{s}}{d \ln k} \simeq 8 \times 10^{-4}$.

\subsection{WMAP and the Inflaton}
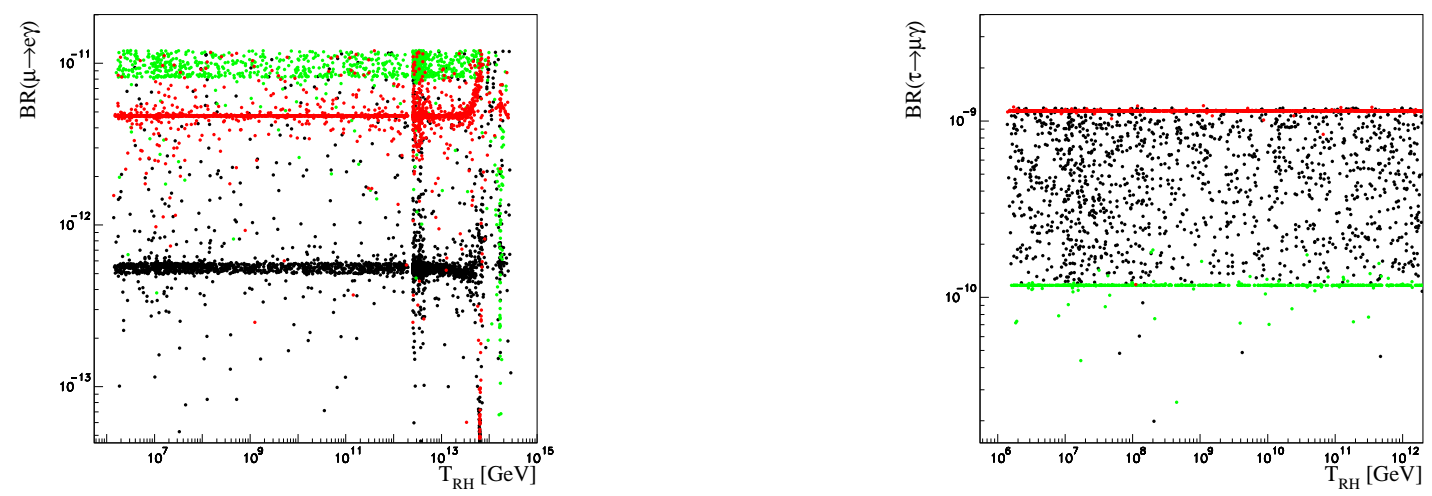

Figure 15: Branching ratios of various Lepton-Flavour-Violation processes in scenaria viewing the Sneutrino as the Inflaton [13].

Despite the agreement, via best fit scenaria, of the WMAP data with inflationary models, unfortunately no information can be obtained at this stage on the nature of the inflaton field. In fact, all the WMAP data imply that at an early stage of our Universe there was an era of exponential expansion, but cannot shed light on what caused this expansion. Although the most popular and successful model of inflation to date involves the inflaton 9], however this is not the only possibility. An exponential expansion of the Universe does not have to be produced by an extra fundamental scalar field. The inflationary phase may be dynamical in nature, without the need of inflaton fields. Such 
issues can only be understood when the full non-equilibrium dynamics of inflationary Universe is at hand.

We mention in passing at this point that within the context of string theory [14, the dilaton can play the rôle of the inflaton field, and that this mode may arise dynamically in some non-critical string scenaria by incorporating properly non-equilibrium dynamics via the so-called Liouville mode [15. The Liouville mode is a world-sheet field [16, which arises from the need for restoration of the conformal invariance of the underlying stringy $\sigma$-model theory, which is broken as a result of a cosmically catastrophic event, such as an initial quantum fluctuation or, in a modern context, the collision between brane Universes, one of which represents our world [17, 18, 19]. In such models, the Liouville field appears with a time-like signature, and hence may be viewed [20, 21] as an (irreversible) target time variable in the system. The irreversibility is guaranteed by world-sheet renormalization-group arguments, given that the zero mode of the Liouville field plays the rôle of a world-sheet scale [20].

Due to its specific coupling with the two-dimensional curvature of the world-sheet of the stringy $\sigma$-model, the Liouville mode contributes linear (in target time) terms in the dilaton excitation of the string spectrum. Linear-in-target-time dilaton cosmology has been discussed in 22, where it was shown that it implies linearly expanding Universes in the Robertson-Walker time ${ }^{1}$ To obtain inflation in such non-critical string scenaria requires more complicated string backgrounds [15, 17, 18, 19, and such models have been discussed in the literature, also in connection with a current-era acceleration of the Universe. We shall come back to such issues in section 6 .

Quite recently, other interesting particle physics scenaria have been suggested [13], in which the inflaton may be the supersymmetric partner of the neutrino (sneutrino). The model is argued to be consistent with the WMAP data, corresponding to a quadratic inflaton potential $V \sim \phi^{2}$. In such scenaria Leptogenesis leads to a prediction for the Lepton Flavour Violation (LFV) of the model which appears consistent with the WMAP constraint on the baryon density at a $3-\sigma$ level: $7.8 \times 10^{-11}<Y_{B}<1.0 \times 10^{-10}$. It must be noted however that the model is not consistent with the WMAP non-zero value of the running of the spectral index $d n_{s} / d \ln k$. However at present the data are too crude for an unambiguous determination of such quantities, and so the authors of [13] used only the $1 \sigma$ level result of that particular measurement to fit their model.

One can calculate in this model LFV branching ratios $B R(\tau \rightarrow \mu \gamma) \lesssim 10^{-9}, B R(\mu \rightarrow$ $e \gamma) \lesssim 10^{-11}, B R(\tau \rightarrow e \gamma)<10^{-7}$, as well as electron and muon dipole moments, which have been argued [13] to be consistent with present experimental limits. The most sensitive

\footnotetext{
${ }^{1}$ In stringy cosmology, the Robertson-Walker frame is defined as the one in which the time in the metric has unit coefficient, but also the Einstein scalar curvature term in the low-energy effective field-theory action has no exponential dilaton terms in front. This requires some redefinition, not only of the time variable, but also of the target space metric background by appropriate exponential dilaton factors [22. Under such redefinitions, an originally linear in target time dilaton, becomes logarithmic in Robertson-Walker time.
} 
experiments to measure such BRs are those involving neutrino oscillations. Note that such BR cannot be measured in the ATLAS/LHC experiment, whose sensitivity to such LFV processes is much poorer $\left(B R(\tau \rightarrow \mu \gamma)_{\text {ATLAS }} \sim 10^{-6}\right)$.

\section{WMAP AND DARK MATTER}

\subsection{Hot and Warm Dark Matter Excluded}

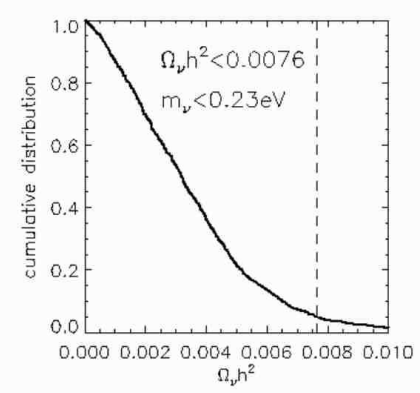

Figure 16: Hot Dark Matter models are excluded in view of the WMAP determination of (low) upper bounds of neutrino masses [3].

The WMAP results on the cosmological parameters discussed previously disfavor strongly Hot Dark Matter (neutrinos), as a result of the new determination of the upper bound on neutrino masses.

The contribution of neutrinos to the energy density of the Universe depends upon the sum of the mass of the light neutrino species [7, 5]:

$$
\Omega_{\nu} h^{2}=\frac{\sum_{i} m_{i}}{94.0 \mathrm{eV}}
$$

where the sum includes neutrino species that are light enough to decouple while still relativistic.

The combined results from WMAP and other experiments [5] on the cumulative likelihood of data as a function of the energy density in neutrinos is given in figure 16. Based on this analysis one may conclude that $\Omega_{\nu} h^{2}<0.0067$ (at $95 \%$ confidence limit). Adding the Lyman $\alpha$ data, the limit weakens slightly [5]:

$$
\Omega_{\nu} h^{2}<0.0076
$$

or equivalently (from (9)): $\sum_{i} m_{\nu_{i}}<0.69 \mathrm{eV}$, where, we repeat again, the sum includes light species of neutrinos. This may then imply an average upper limit on electron neu- 
trino mass $<m_{\nu}>_{e}<0.23 \mathrm{eV}$. The upper bound on the relevant densities (10) strongly disfavours Hot Dark Matter scenarios.
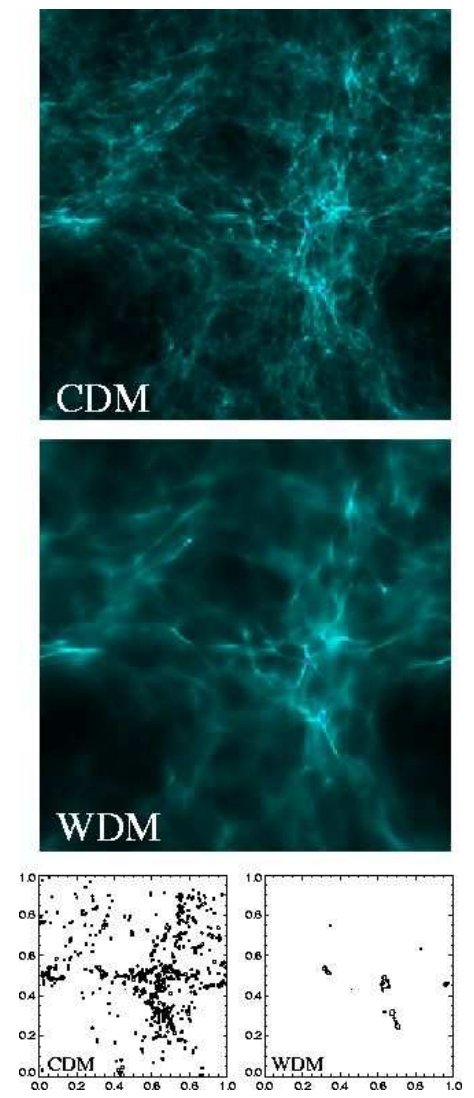

Figure 17: Numerical simulations for structure formation in Cold Dark Matter (CDM) and Warm Dark Matter (WDM) models with mass $m_{X}=10 \mathrm{KeV}$ [23]. Upper two figures: The projected gas distribution in the CDM (top) and the WDM (midde) simulations at $z=20$. Lower bottom figures: the distribution of dark halos with mass greater than $10^{5} M_{\odot}$

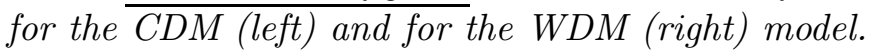

Another important result of WMAP is the evidence for early re-ionization of the Universe at redshifts $z \simeq 20$. If one assumes that structure formation is responsible for re-ionization, then such early reionization periods are compatible only for high values of the masses $m_{X}$ of Warm Dark Matter. Specifically, one can exclude models with $m_{X} \leq 10$ $\mathrm{KeV}$ [5, 23] based on numerical simulations of structure formation for such models. Such simulations (c.f. figure 17) imply that dominant structure formation responsible for reionization, for Warm Dark Matter candidates with $m_{X} \leq 10 \mathrm{KeV}$, occurs at much smaller 
$z$ than those observed by WMAP. In view of this, one can therefore exclude popular particle physics models employing light gravitinos $\left(m_{X} \simeq 0.5 \mathrm{KeV}\right)$ as the Warm Dark Matter candidate.

It should be noted at this stage that such structure formation arguments can only place a lower bound on the mass of the Warm Dark Matter candidate. The reader should bear in mind that Warm Dark Matter with masses $m_{X} \geq 100 \mathrm{KeV}$ becomes indistinguishable from Cold Dark Matter, as far as structure formation is concerned.

\subsection{Cold Dark Matter in Supersymmetric Models: Neutralino}

After the exclusion of Hot and Warm Dark Matter, the only type of Dark matter that remains consistent with the recent WMAP results [5] is the Cold Dark Matter, which in general may consist of axions, superheavy particles (with masses $\sim 10^{14 \pm 5} \mathrm{GeV}$ ) 24,25 , and stable supersymmetric partners.

Indeed, one of the major and rather unexpected predictions of Supersymmetry (SUSY), broken at low energies $M_{S U S Y} \approx \mathcal{O}(1 \mathrm{TeV})$, while $R$-parity is conserved, is the existence of a stable, neutral particle, the lightest neutralino $\left(\chi_{1}^{0}\right)$, referred to as the lightest supersymmetric particle (LSP) 6]. Such particle is an ideal candidate for the Cold Dark Matter in the Universe [6]. Such a prediction fits well with the fact that SUSY is not only indispensable in constructing consistent string theories, but it also seems unavoidable at low energies $(\sim 1 \mathrm{TeV})$ if the gauge hierarchy problem is to be resolved. Such a resolution provides a measure of the SUSY breaking scale $M_{S U S Y} \approx \mathcal{O}(1 \mathrm{TeV})$. There is indirect evidence for such a low-energy supersymmetry breaking scale, from the unification of the gauge couplings [26] and from the apparent lightness of the Higgs boson as determined from precise electroweak measurements, mainly at LEP [27].

This type of Cold Dark Matter will be our focus from now on, in association with the recent results from WMAP on relic densities [3, 5]. The WMAP results for the baryon and matter densities (including dark matter) are given in the table of figure10. After combining the WMAP data with other existing data, one obtains the following final values:

$$
\begin{aligned}
& \Omega_{m} h^{2}=0.135_{-0.009}^{+0.008} \text { (matter) } \\
& \Omega_{b} h^{2}=0.0224 \pm 0.0009 \text { (baryons). }
\end{aligned}
$$

Hence, assuming that CDM is given by the difference of these two, one has

$$
\Omega_{C D M} h^{2}=0.1126_{-0.0181}^{+0.0161}, \quad(2-\sigma \quad \text { level }) .
$$

As mentioned already, in supersymmetric (SUSY) theories the favorite candidate for CDM is the lightest of the Neutralinos $\chi_{1}^{0}$ (SUSY CDM), which is stable, as being the Lightest SUSY particle (LSP) ${ }^{2}$. From the above-described WMAP results, then, assuming

\footnotetext{
${ }^{2}$ There are cases where the stau or the sneutrino can be the lightest supersymmetric particles. These cases are not favoured [28] and hence are not considered.
} 
$\Omega_{C D M} \simeq \Omega_{\chi}$, we can infer stringent limits for the neutralino $\chi$ relic density:

$$
0.094<\Omega_{\chi} h^{2}<0.129, \quad(2-\sigma \quad \text { level })
$$

It is important to notice that in this inequality only the upper limit is rigorous. The lower Limit is optional, given that there might (and probably do) exist other contributions to the overall (dark) matter density.

The constraints in SUSY models we shall review in this work come precisely from the use of this constraint, together with other results mainly from the anomalous magnetic moment of the muon, $g_{\mu}-2$, the decay $b \rightarrow s \gamma$, as well as LEP-induced Higgs mass lower limits. Specifically, we shall constrain the minimal SUSY Standard Model (MSSM) with universal values for the masses of scalars $m_{0}$ the gauginos $m_{1 / 2}$ and trilinear soft couplings $A_{0}$ at the GUT scale. Furthemore Electroweak Symmetry Breaking (EWSB) is assumed. This is the so-called Constrained MSSM (CMSSM). For the purposes of this review we shall concentrate on plots $\left(m_{0}, m_{1 / 2}\right)$ for the minimal supergravity model (mSUGRA) [29, 30, in which such CMSSM are embedded.

It is imperative to notice that all the constraints we shall discuss in this review are highly model dependent and the results cannot be extrapolated to non-minimal models. But most of the analysis can be extrapolated to such models, with possibly different results. In what follows, therefore, for definiteness we shall concentrate exclusively to mSUGRA models. We shall not discuss non-minimal models in this article.

\section{WMAP AND SUSY CDM}

\subsection{CMSSM/mSUGRA: Basic Features}

Before embarking into a detailed analysis of the constraints of the minimal supersymmetric standard model embedded in a minimal supergravity model (CMSSM) 29], we consider it useful to outline the basic features of these models, which will be used in this review.

The embedding of SUSY models into the minimal supergravity (mSUGRA) model implies that there are five independent parameters: Three of them, the scalar and gaugino masses $m_{0}, m_{1 / 2}$ as well as the trilinear soft coupling $A_{0}=$, at the unification scale, set the size of the Supersymmetry breaking scale. In addition one can consider as input parameter $\tan \beta=\frac{\left\langle H_{2}\right\rangle}{\left\langle H_{1}\right\rangle}$, the ratio of the v.e.v's of the Higgses $H_{2}$ and $H_{1}$ giving masses to up and down quarks respectively. The sign ( signature) of the Higgsino mixing parameter $\mu$ is also an input but not its size which is determined from the Higgs potential minimization condition

$$
\mu^{2}+\frac{M_{Z}^{2}}{2}=\frac{m_{H_{1}}^{2}-m_{H_{2}}^{2} \tan \beta^{2}}{\tan \beta^{2}-1}+\frac{\Sigma_{1}-\Sigma_{2} \tan \beta^{2}}{\tan \beta^{2}-1} .
$$


In (14) $M_{Z}$ is actually the running $\mathrm{Z}$ - boson mass and $m_{H_{1,2}}$ the soft masses of the Higgses $H_{2}$ and $H_{1}$ respectively. $\Sigma_{1,2}$ on the r.h.s of this equation are contributions arising from loop corrections of the effective potential 31, 32.

The parameter space of mSUGRA can be effectively described in terms of two branches as can be seen by exploring the minimization condition (14). Following the discussion in [33] we can demonstrate this analytically ignoring, for simplicity, the coupling of the $b$ quark. In this case (14) can be approximated by [32] :

$$
C_{1} m_{0}^{2}+C_{3} m_{1 / 2}^{\prime 2}+C_{2}^{\prime} A_{0}^{2}+\Delta \mu_{\text {loop }}^{2}=\mu^{2}+\frac{1}{2} M_{Z}^{2}
$$

where

$$
m_{1 / 2}^{\prime} \equiv m_{1 / 2}+\frac{1}{2} A_{0} \frac{C_{4}}{C_{3}}, \quad C_{2}^{\prime}=C_{2}-\frac{1}{4} \frac{C_{4}^{2}}{C_{3}}
$$

and

$$
\begin{aligned}
& C_{1}=\frac{1}{t^{2}-1}\left(1-\frac{3 D_{0}-1}{2} t^{2}\right), \quad C_{2}=\frac{t^{2}}{t^{2}-1} k, \\
& C_{3}=\frac{1}{t^{2}-1}\left(g-t^{2} e\right), \quad C_{4}=-\frac{t^{2}}{t^{2}-1} f, \Delta \mu_{\text {loop }}^{2}=\frac{\Sigma_{1}-t^{2} \Sigma_{2}}{t^{2}-1} .
\end{aligned}
$$

$\Delta \mu_{\text {loop }}$ denote the loop correction to the parameter $\mu, t=\tan \beta, \Sigma_{1,2}$ were defined before, the functions $e, f, g, k$ are as defined in 34], and $D_{0}=1-\left(m_{t} / m_{f}\right)^{2}$ where $m_{f} \simeq 200 \sin \beta$ $\mathrm{GeV}$.

- Ellipsoidal Branch (EB) of Radiative Symmetry Breaking :

For small to moderate values of $\tan \beta \lesssim 7$, the loop corrections are typically small, and the renormalization group analysis shows that both $C_{2}^{\prime}>0$ and $C_{3}>0$. In this case one finds $C_{1}>0$ independently of any scale choice $Q$ for the radiative electroweak symmetry breaking. Then, one finds that the radiative symmetry breaking constraint demands that the allowed set of soft parameters $m_{0}$ and $m_{1 / 2}^{\prime}$ lie, for a given value of $\mu$, on the surface of an Ellipsoid. This places upper bounds on the sparticle masses for a given value of $\Phi \equiv \mu^{2} / M_{Z}^{2}+1 / 4$. This is the ellipsoidal branch (EB) of the Electroweak Radiative Symmetry Breaking (EWRSB).

Although this is always true for values $\tan \beta \lesssim 7$, it does not necessarily hold for larger values $\tan \beta \gtrsim 7$. In the latter case $m_{0}$ and $m_{1 / 2}^{\prime}$ may lie on the surface of a Hyperboloid. This situation, which peresents phenomenological interest, is described in more detail below [32].

- Hyperbolic Branch (HB) of Radiative Symmetry Breaking:

For large values of $\tan \beta \gtrsim 7$, the loop corrections to $\mu$ are significant and $\mu$ also exhibits a significant variation with varying the scale $Q$. If we choose the running 
scale at $Q_{0}$ where the loop corrections are minimized, or even vanish, the loop corrections to the r.h.s of (14) can be ommited and all quantities appearing in eq. (14) should be considered at this scale. Usually the scale $Q_{0}$ is the geometric average of the stop masses which give the largest contributions to $\Sigma_{1,2}$. In some regions of the parameter space it may occur that the sign of $C_{1}$ is flipped, i.e. $\operatorname{sign}\left(C_{1}\left(Q_{0}\right)\right)=-1$, so that the minimization condition receives the form

$$
\frac{m_{1 / 2}^{\prime}{ }^{2}}{\alpha^{2}\left(Q_{0}\right)}-\frac{m_{0}^{2}}{\beta^{2}\left(Q_{0}\right)} \simeq \pm 1 .
$$

In (18) $\alpha=\frac{\left|\left(\Phi_{0}+\frac{1}{4}\right) M_{Z}^{2}-C_{2}^{\prime} A_{0}^{2}\right|}{\left|C_{3}\right|}, \beta=\frac{\left|\left(\Phi_{0}+\frac{1}{4}\right) M_{Z}^{2}-C_{2}^{\prime} A_{0}^{2}\right|}{\left|C_{1}\right|}$, and the \pm sign is determined by the condition $\operatorname{sign}\left(\left(\Phi+\frac{1}{4}\right) M_{Z}^{2}-C_{2}^{\prime} A_{0}^{2}\right)= \pm 1$. It is apparent from (18) that unlike the previous case $\left(m_{0}, m_{1 / 2}^{\prime}\right)$ lie now on the surface of a hyperboloid and hence the name Hyperbolic Branch (HB).

What is interesting in the $\mathrm{HB}$ case it the fact that $m_{0}$ and or $m_{1 / 2}$ can become very large while much smaller values for $\mu$. A subset of HB is the so called high zone. In this case EWSB can occur in regions where $m_{0}$ and $m_{1 / 2}$ can be in the several TeV range, with much smaller values for the parameter $\mu$ which however is much larger than $M_{Z}$. This has important consequences for phenomenology as we shall see. In this zone the lightest of the neutralinos, $\chi_{1}$, is almost a Higgsino having mass $\mu$. This is called inversion phenomenon since the LSP is a Higgsino rather a Bino.

Except the high zone where the inversion phenomenon takes place the HB includes the so called Focus Point (FP) region [35, 36, which is defined as a region in which some renormalization group $(\mathrm{RG})$ trajectories intersect (FP region would be only a point, were it not for threshold effects which smear it out). We stress that the FP is not a fixed point of the RG. The FP region is a subset of the HB limited to relatively low values of $m_{1 / 2}$ and values of $\mu$ close to the electroweak scale, $M_{Z}$, while $m_{0}$ can be a few TeV but not as large as in the high zone due to the constraints imposed by the EWSB condition. The LSP neutralino in this region is a mixture of Bino and Higgsino and the Higgsino impurity allowes for rapid s-channel LSP annihilations, resulting to low neutralino relic densities at experimentally acceptable levels.

From the previous discussion concerning the HB region it is obvious that the crucial property characterizing this region is the inversion of the sign of $C_{1}$. For large $\tan \beta$ ( $\tan \beta \gtrsim 7$ ), the sign of $C_{1}$ is mainly driven by that of the quantity $3 D_{0}-1$ as is seen from eq. (17). In order for $C_{1}$ to be negative $3 D_{0}-1$ should be positive which with the approximations adopted so far entails to

$$
\sin \beta>\sqrt{\frac{3}{2}} \frac{m_{t}}{200 \mathrm{GeV}} \approx \frac{m_{t}}{163.3 \mathrm{GeV}}
$$


Even from this crude approximation it is apparent the central role the value of the running top mass $m_{t}$ it plays for the location of the Hyperbolic Branch. If it happens $m_{t}>$ $163.3 \mathrm{GeV}$, at the scale $Q_{0}$, the HB is never reached. Thus the preference towards smaller values of the top quark mass, close to the lowest experimental limit, for the location of the HB is manifest from this discussion. Therefore the HB region is sensitive to variations of the parameters of the theory which may induce a shift of the theoretically determined $m_{t}$ to higher values by 1-2\%. It then becomes evident that two loop RGE effects to Yukawa couplings, radiative corrections to the top mass and supersymmetric corrections to the bottom Yukawa coupling, which are important for large values of $\tan \beta$, as well as low energy threshold effects to the strong coupling constant, which greatly affect the RGE running of the parameters of the theory and hence low energy predictions, must be duly taken into account for a correct and unambiguous phenomenological description of this region. These subtleties may drastically affect the conclusions reached in various analyses and this is the reason this phenomenologically interesting region is sometimes considered as being fine tuned.

\subsection{Focusing The Cosmologically Relevant Regions}

Having described the two principal regions, EB and HB, with different phenomenolological characteristics each, we now pass to briefly outline the regions which posses special interest as far as CDM is concerned.

In the EB region the EWSB condition drives the parameter $\mu$ to be of the same order of magnitude with $m_{0}, m_{1 / 2}$. In the bulk of this region the lightest of the neutralinos, $\chi_{1}^{0}$, is mostly a bino except a region with small $m_{1 / 2}$ in which $\chi_{1}^{0}$ has a sizeable Higgsino component. This region is however experimentally ruled out by $b \rightarrow s \gamma$ data and light chargino searches. Excluding this region we focus our attention to the following subregions which possess special features as far as Cosmology and particle physics is concerned. The first is the stau coannihilation region in which $\chi_{1}^{0}$ is almost degenerate in mass, but heavier, with the light $\tau$-slepton. When this happens the calculation of the neutralino CDM requires the coannihilations $\chi_{1}^{0}-\tilde{\tau}$ and $\tilde{\tau}-\tilde{\tau}$ [37, 38, 39]. In this region the mass of $\chi_{1}^{0}$ is allowed to be extended up to $500 \mathrm{GeV}$ assuming that the WMAP data are respected. The second region is characterized by large $\tan \beta \gtrsim 45$, and concerns the region where $2 m_{\chi_{1}^{0}} \simeq$ $m_{A}$ where $m_{A}$ is the pseudoscalar Higgs mass. In this region s-channel pseudoscalar annihilation to a $b-\bar{b}$ or a $\tau-\bar{\tau}$ pair becomes important in opening up extended regions compatible with WMAP data. Interestingly enough this large $\tan \beta$ region yields high values, $\simeq 10^{-8,9} \mathrm{pb}$ for the elastic $\chi_{1}^{0}$ - nucleon cross section close to the sensitivity limits of on going and future direct dark matter experiments. We postpone a more detailed discussion of this issue later on in this review.

Moving to the $\mathrm{HB}$, in general the scalar $m_{0}$ and gaugino $m_{1 / 2}$ masses can get very large, which implies that the squarks and sleptons can be very heavy, with masses that 
lie in the several $\mathrm{TeV}$ range. The feature of the large $m_{0}$ mass is also shared by the focus point region of mSUGRA models [35, 36]. Consider the high zone of the HB, in which $m_{1 / 2} \gg \mu \gg M_{Z}$. In this scenario one finds [32, 33] that the two lightest neutralino states, $\chi_{1}^{0}, \chi_{2}^{0}$ and the light chargino state $\chi_{1}^{ \pm}$are essentially degenerate with mass $\sim \mu$. Theis masses range from several hundreds $\mathrm{GeV}$ to above $\mathrm{TeV}$ while their mass differences are much smaller in the range 1-10 GeV.To first order they are degenerate all having a mass $\mu$ but this degeneracy is lifted by small corrections $\mathcal{O}\left(\frac{M_{Z}}{\mu}\right)$ entailing to a mass spectrum

$$
m_{\chi_{1}^{0}}<m_{\chi_{1}^{ \pm}}<m_{\chi_{2}^{0}}
$$

with $\chi_{1}^{ \pm}$the lightest chargino states and $\chi_{2}^{0}$ the next to lightest neutralino. As mentioned earlier in this region the inversion phenomenon takes place given that the lightest neutralino switches from being mostly a Bino to being a higgsino.

The inversion phenomenon has dramatic effects on the nature of the particle spectrum and SUSY phenomenology in this HB. Indeed, in mSUGRA one naturally has coannihilation with the sleptons when the neutralino mass extends to masses beyond $150-200 \mathrm{GeV}$ with processes of the type $\chi \tilde{\ell}_{R}^{a} \rightarrow \ell^{a} \gamma, \ell^{a} Z, \ell^{a} h, \tilde{\ell}_{R}^{a} \tilde{\ell}_{R}^{b} \rightarrow \ell^{a} \ell^{b}$, and $\tilde{\ell}_{R}^{a} \tilde{\ell}_{R}^{b *} \rightarrow$ $\ell^{a} \bar{\ell}^{b}, \gamma \gamma, \gamma Z, Z Z, W^{+} W^{-}, h h$ where $\tilde{l}$ is essentially a $\tilde{\tau}$. Remarkably the relic density constraints can be satisfied on the hyperbolic branch also by coannihilation. However, on the HB the coannihilation is of an entirely different nature as compared with the stau coannihilations discussed previously: instead of a neutralino-stau co-annihilation, and stau stau in the HB one has co-annihilation processes involving the second lightest neutralino and chargino states [40], $\chi_{1}^{0}-\chi_{1}^{ \pm}$, followed by $\chi_{1}^{0}-\chi_{2}^{0}, \chi_{1}^{+}-\chi_{1}^{-}, \chi_{1}^{ \pm}-\chi_{2}^{0}$. Some of the dominant processes that contribute to the above coannihilation processes are [40]

$$
\begin{array}{r}
\chi_{1}^{0} \chi_{1}^{+}, \chi_{2}^{0} \chi_{1}^{+} \rightarrow u_{i} \bar{d}_{i}, \bar{e}_{i} \nu_{i}, A W^{+}, Z W^{+}, W^{+} h \\
\chi_{1}^{+} \chi_{1}^{-}, \chi_{1}^{0} \chi_{2}^{0} \rightarrow u_{i} \bar{u}_{i}, d_{i} \bar{d}_{i}, W^{+} W^{-}
\end{array}
$$

Since the mass difference between the states $\chi_{1}^{+}$and $\chi_{1}^{0}$ is the smallest the $\chi_{1}^{0} \chi_{1}^{+}$coannihilation dominates. In such cases, the masses $m_{0} m_{1 / 2}$ may be pushed beyond $10 \mathrm{TeV}$, so that squarks and sleptons can get masses up to several TeV, i.e. beyond detectability limits of immediate future accelerators such as LHC.

Except the high zone the other interesting region belonging to the $\mathrm{HB}$ is the Focus Point (FP). As already mentioned in the previous chapter unlike the high zone this is characterized by $m_{0}$ in the few $\mathrm{TeV}$ range, low values of $m_{1} 1 / 2<<m_{0}$ and rather small values of $\mu$ close to $M_{Z}$. The LSP neutralino in this case is a mixture of Bino and Higgsino and its Higgsino impurity is adequate to give rize to rapid s-channel LSP annihilations so that the neutralino relic density is kept low at experimentally acceptable values. Since $\mu$ is small the lightest chargino may be lighter than $500 \mathrm{GeV}$ and the FP region may be accessible to future $\mathrm{TeV}$ scale colliders. Also due to the relative smallnes of $m_{1 / 2}$ in this 
region gluino pair production may occur at a high rate making the FP region accessible at LHC energies.

As we remarked before although the HB may be viewed as fine tuned, nevertheless recent studies [41, based on a $\chi^{2}$ analysis, have indicated that the WMAP data, when combined with data on $b \rightarrow s \gamma$ and $g_{\mu}-2$, seem to favour the Focus Point HB region and the large $\tan \beta$ neutralino resonance annihilation of mSUGRA.

\subsection{SUSY CDM in the pre-WMAP era and the importance of the muon's anomaly}

Before embarking onto a discussion on the constraints on SUSY CDM implied by the recent WMAP data on relic densities, we consider it as instructive to review phenomenological works before the publication of these data.

The issue of the Dark Matter has attracted the interest of many physicists and in the literature there are numerous theoretical works dealing with this issue [42, 43, 44, 45, 46, 47, 48, 49, 50, 6, 40. Departures from the minimal mSUGRA models and studies including non-universalities [51, CP - violating effects [52] as well as efforts related to Yukawa unification [38, 53] have been pursued. Phenomenological studies including the effects of variations of the WIMP velocities on the detection rate have also been considered [54] and regions where coannihilation phenomena are important have been analyzed [55. 40, 37, 38, 39, 56, 57.

The importance of the smallness of the Dark Matter (DM) relic density in constraining supersymmetric predictions had been pointed out in the past [58, 59, 60, 61, paying special attention at the to the large $\tan \beta$ regime. In this region the neutralino $\left(\chi_{1}^{0}\right)$ pair annihilation through $s$-channel pseudo-scalar Higgs boson $(A)$ exchange, leads to an enhanced annihilation cross sections reducing significantly the relic density 62. The importance of this mechanism, in conjunction with the cosmological data which favour small values of the DM relic density, has been stressed in [58, 59].

In previous analyses regarding DM direct searches [59], we had stressed that the contribution of the $C P$-even Higgs bosons exchange to the LSP-nucleon scattering cross sections increases with $\tan \beta$. Therefore in the large $\tan \beta$ region one obtains the highest possible rates for the direct DM searches and the smallest LSP relic densities. Similar results are presented in Ref. 63. Early studies on small $\Omega_{\chi} h^{2} \lesssim 0.18$, taking into account constraints from the anomalous magnetic moment of the muon, have been carried out in [61, based on the mSUGRA model. The results from such analyses are depicted in figure 18

The pre-WMAP situation 64, involving the efforts of other groups, concerning prospects for detecting SUSY is summarized in figure 19, where we also give some benchmark scenaria 65.

Undoubtedly one of the most significant experimental results of the last years is the measurement of the anomalous magnetic moment of the muon 66. Deviation of its mea- 

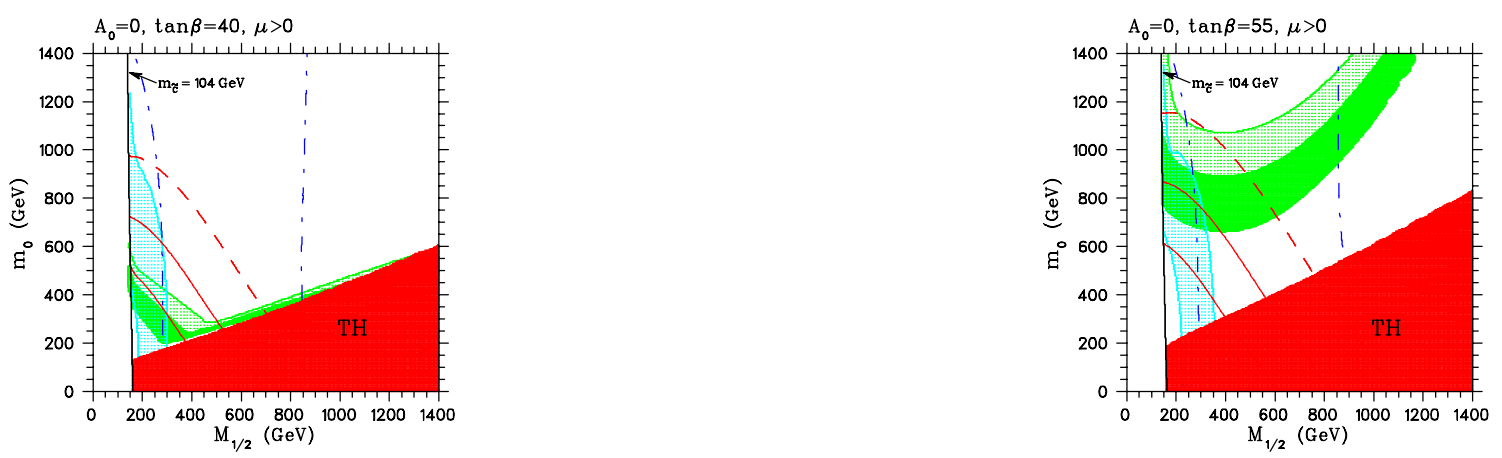

Figure 18: mSUGRA/CMSSM constraints before WMAP [58, 60]. The Mass of the top quark is taken $175 \mathrm{GeV}$. Dark Green shaded areas correspond to neutralino relic densities in the range: $0.08<\Omega_{\chi} h^{2}<0.18$, whilst light green areas correspond to $0.18<\Omega_{\chi} h^{2}<$ 0.30. The Solid Red Line indicates constraints implied by the anomalous magnetic moment of the muon $a_{\mu}^{\text {SUSY }}=(306 \pm 106) \times 10^{-11}$. The Dashed red line indicates the boundary of the region for which the lower bound is moved to $2 \sigma$ limit. The Dashed Blue lines correspond to constraints implied by the Higgs mass $113.5 \mathrm{GeV}<m_{\text {Higgs }}<117.0 \mathrm{GeV}$. Finally Cyan shaded region on the right are excluded due to $b \rightarrow s \gamma$ constraint.

sured value from the Standard Model (SM) predictions is evidence for new physics with Supersymmetry being the prominent candidate to play that role. Adopting Supersymmetry as the most natural extension of the SM, such deviations may be explained and impose at the same time severe constraints on the predictions of the available SUSY models by putting upper bounds on sparticle masses. Therefore knowledge of the value of $g_{\mu}-2$ is of paramount importance for Supersymmetry and in particular for the fate of models including heavy sparticles in their mass spectrum, as for instance those belonging to the Hyperbolic Branch.

Unfortunately the situation concerning the anomalous magnetric moment is not clear as some theoretical uncertainties remain unsettled as yet. At present, there are two theoretical estimates for the difference of the experimentally measured [66] value of $a_{\mu}=\left(g_{\mu}-2\right) / 2$ from the theoretically calculated one within the SM [67,

- Estimate (I) $a_{\mu}^{\exp }-a_{\mu}^{\mathrm{SM}}=1.7(14.2) \times 10^{-10} \quad\left[0.4(15.5) \times 10^{-10}\right]$

- Estimate (II) $a_{\mu}^{\exp }-a_{\mu}^{\mathrm{SM}}=24.1(14.0) \times 10^{-10} \quad\left[22.8(15.3) \times 10^{-10}\right]$

In (I) the $\tau$-decay data are used in conjuction with Current Algebra while in (II) the $e^{-} e^{+} \rightarrow$ Hadrons data are used in order to extract the photon vacuum polarization which enters into the calculation of $g_{\mu}-2$. Within square brackets are the most updated values 

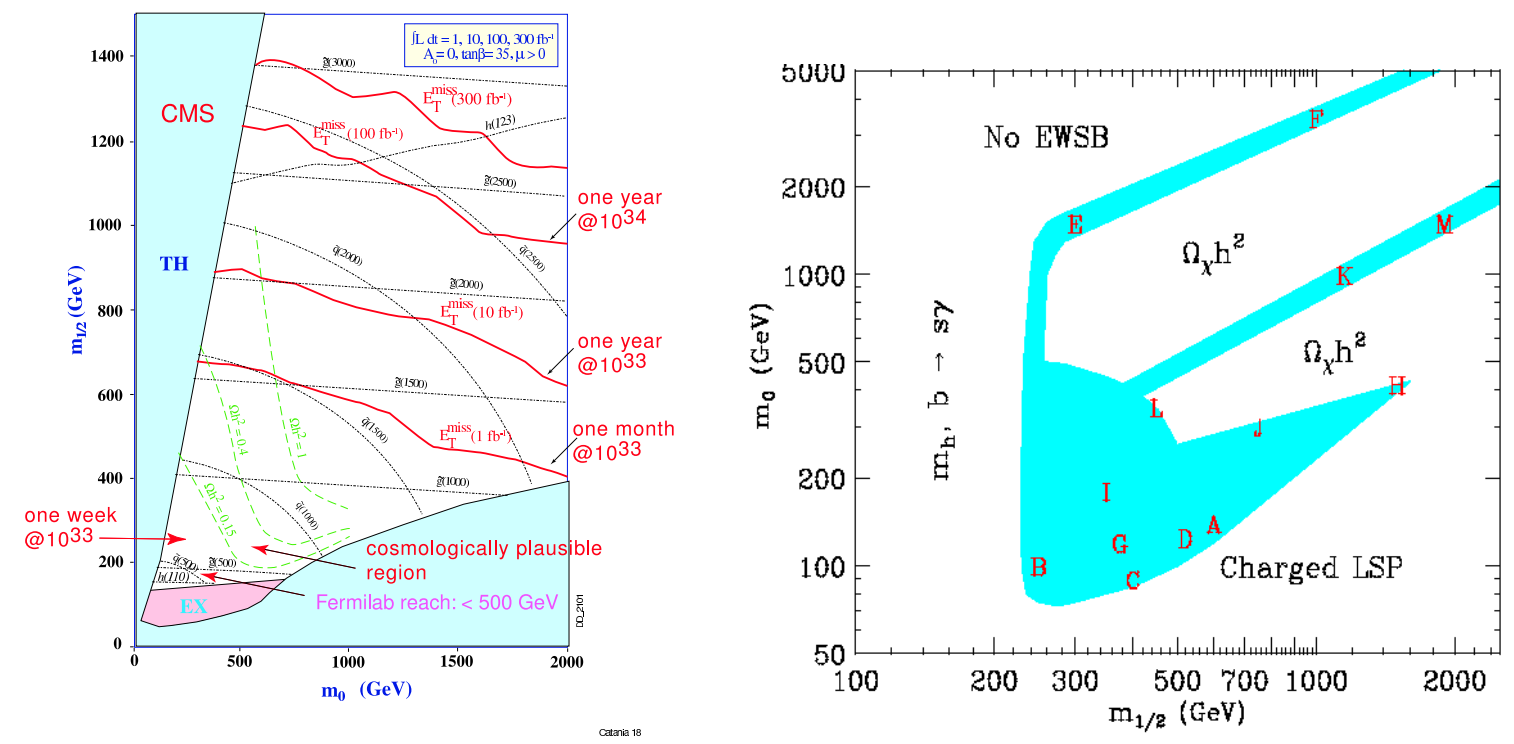

Figure 19: Left figure: SUSY detection prospects before WMAP data [64], explorable at LHC using missing energy and jet signature. Right Figure: Post-LEP Benchmark SUSY scenaria [65]. Benchmark points have $\Omega_{\chi} h^{2}$ larger than the bounds implies by the WMAP data.

of Ref. [67 ${ }^{3}$. Estimate (I) is considered less reliable since it carries additional systematic uncertainties and for this reason in many studies only the Estimate (II) is adopted. Estimate (II) includes the contributions of additional scalar mesons not taken into account in previous calculations.

In order to get an idea of how important the data on the muon anomaly might be we quote Ref. 33. where both estimates have been used. If Estimate (II) is used at a $1.5 \sigma$ range much of the $\mathrm{HB}$ and all of the inversion region can be eliminated. In that case the usually explored region of SUSY in the EB is the only one that survives, which, as we shall discuss below, can be severely constrained by means of the recent WMAP data.

On the other hand, Estimate (I), essentially implies no difference from the SM value, and hence, if adopted, leaves the HB, and hence its high zone (inversion region), intact. In such a case, SUSY may not be detectable at colliders, at least in the context of the mSUGRA model, but may be detectable in some direct dark matter searches, to which we shall turn to later in the article.

For the above reasons, it is therefore imperative to determine unambiguously the muon anomalous magnetic moment $g_{\mu}-2$ by reducing the errors in the leading order hadronic

\footnotetext{
${ }^{3}$ Due to the rapid updates concerning $g_{\mu}-2$ the values of $a_{\mu}^{\exp }-a_{\mu}^{\mathrm{SM}}$ used in previous works quoted in this article may differ from those appearing above.
} 
contribution, experimentally, and improving the theoretical computations within the standard model. In view of its importance for SUSY searches, it should also be necessary to have further experiments in the future, that could provide independent checks of the measured muon magnetic moment by the E821 experiment [6].

\subsection{WMAP and SUSY constraints}

\subsubsection{Constraints in the EB}

After the first year of running of the WMAP satellite [3], the situation concerning mSUGRA model constraints in the EB of radiative symmetry breaking parameter space, has changed dramatically 68, 69. The constraints on the neutralino relic density (12, 13), implied by the best fit Universe model of the WMAP data combined with other experiments [3, 5], and with other constraints such as the post-LEP Higgs mass limits, $b \rightarrow s \gamma$ and $g_{\mu}-2$, have restricted severely the available parameter space of the EB.

Some important remarks are in order at this stage:

In Ref. 69] in calculating the $\chi_{1}^{0}$ relic abundance, the authors solve the Boltzmann equation numerically using the machinery outlined in Ref. [58. In this calculation the coannihilation effects, in regions where $\tilde{\tau}_{R}$ approaches in mass the LSP, which is a high purity Bino, are properly taken into account.

The details of the procedure in calculating the spectrum of the CMSSM can be found elsewhere [70, 61, 60. Here we shall only briefly refer to some subtleties which turn out to be essential for a correct determination of the Higgsino, $\mu$, and Higgs, $m_{3}^{2}$, mixing parameters, which greatly affect the Neutralino, Chargino and Higgs mass spectra, especially in the region of large $\tan \beta$. This region is of particular phenomenological interest as we discussed in previous sections. $\mu$ and $m_{3}^{2}$ are determined by minimizing the one-loop corrected effective potential [31]. For large $\tan \beta$ the derivatives of the effective potential with respect the Higgs fields, which enter into the minimization conditions, are plagued by terms which are large and hence potentially dangerous, making the perturbative treatment untrustworthy. In order to minimize the large $\tan \beta$ corrections we had better calculate the effective potential using as reference scale the average stop scale $Q_{\tilde{t}} \simeq \sqrt{m_{\tilde{t}_{1}} m_{\tilde{t}_{2}}}[71$. At this scale these terms are small and hence perturbatively trusted.

The proceedure in solving the renormalization group equations for masses and couplings follows essentially that of Ref. [72]. The low energy threshold corrections to the strong coupling constant are duly taken into account and the unification scale $M_{G U T}$ is defined at the point where the gauge couplings $\alpha_{1,2}$ meet. No unification of the gauge coupling constant $\alpha_{3}$ is enforced with $\alpha_{1,2}$ at $M_{G U T}$ as this usually results to too high values for the strong coupling constant $\alpha_{s}$ at $M_{Z}$. Therefore to comply with the experimentally value for $\alpha_{s}\left(M_{Z}\right)$ we take it as input and thus the value of $\alpha_{3}$ at the unification scale is an output rather than an input. 
A significant correction, which drastically affects the numerical proceedure arises from the gluino-sbottom and chargino-stop corrections to the bottom quark Yukawa coupling $h_{b}$ [73, 74, 72, 75]. The proper resummation of these corrections is important for a correct determination of $h_{b}$ [76, 77] and this has been taken into account.

Seeking a precise determination of the Higgs boson mass the dominant two-loop corrections to this have been included [78. Concerning the calculation of the $b \rightarrow s \gamma$ branching ratio, the important contributions beyond the leading order, especially for large $\tan \beta$, have been taken into account [79].

There have been two independent groups working on this update of the CMSSM in light of the WMAP data, with similar results 68, 69. and below we summarize the results of their analysis in figures 2021 for some typical values of the parameters $\tan \beta$ and signature of $\mu$.

As mentioned in previous sections the region designated as large $\tan \beta$ has particular phenomenological interest. In this region the dominant neutralino annihilation mechanism is through s - channel annihilation of the pseudoscalar Higgs boson (A), $\chi_{1}^{0} \chi_{1}^{0} \stackrel{A}{\rightarrow} b \bar{b}$ or $\tau \bar{\tau}$. The reason is that by increasing $\tan \beta$ the mass $m_{A}$ decreases, while the neutralino mass remains almost constant, if the other parameters are kept fixed. Thus the pseudoscalar Higgs mass $m_{A}$ is expected eventually to enter into the pole regime in which $m_{A}=$ $2 m_{\chi_{1}^{0}}$. At the same time the pseudoscalar Higgs couplings with $\tau \bar{\tau}, b \bar{b}$ are large as being proportional to $\tan \beta$. Thus although the $\chi_{1}^{0}$ is a high purity bino, which does not couple to A, its small Higgsino component, which does couple, can give sizeable effects due to the largeness of the $A b \bar{b}$ and $A \tau \bar{\tau}$ couplings. Both effects result to enhanced annihilation cross sections dominating the process and hence to small neutralino relic densities [69] over a broad region of the parameter space characterized by large values $\tan \beta$ values. From this discussion it becomes obvious that for a correct determination of the relic density, in the large $\tan \beta$ region, an unambiguous and reliable determination of the $A$-mass, $m_{A}$, is required. In the CMSSM, $m_{A}$ is not a free parameter but is determined once the other parameters are given. $m_{A}$ depends sensitively on the Higgs mixing parameter, $m_{3}^{2}$, which is determined from the minimization conditions of the effective potential, and for its calculation all 1 - loop corrections must be taken into account. It is found [80] that the handy calculation of the pseudoscalar mass through the second derivatives of the effective potential is scale independent and approaches the pole mass to better than $2 \%$ provided that one includes the corrections of all particles, especially those of Charginos and Neutralinos, in addition to the leading corrections of the third generation sfermions.

The analysis of [69] is concentrated on large values of $\tan \beta$ for reasons mentioned above. It is found (see the right panel of the lower part of figure 21) that for such values, a region opens up within which the relic density is cosmologically allowed. This is due to the pair annihilation of the neutralinos through the pseudo-scalar Higgs exchange in the $s$-channel. In this region the ratio $m_{A} / 2 m_{\chi_{1}^{0}}$ approaches unity and the pseudo-scalar 
exchange dominates as explained above. It is for this reason that we give special emphasis to this particular mechanism which opens up for large $\tan \beta$ and delineates cosmologically allowed domains of small relic densities and large elastic neutralino - nucleon cross sections. In this case the lower bound put by the $\left(g_{\mu}-2\right)$ data cuts the cosmologically allowed region which would otherwise allow for very large values of $m_{0}, m_{1 / 2}$. 

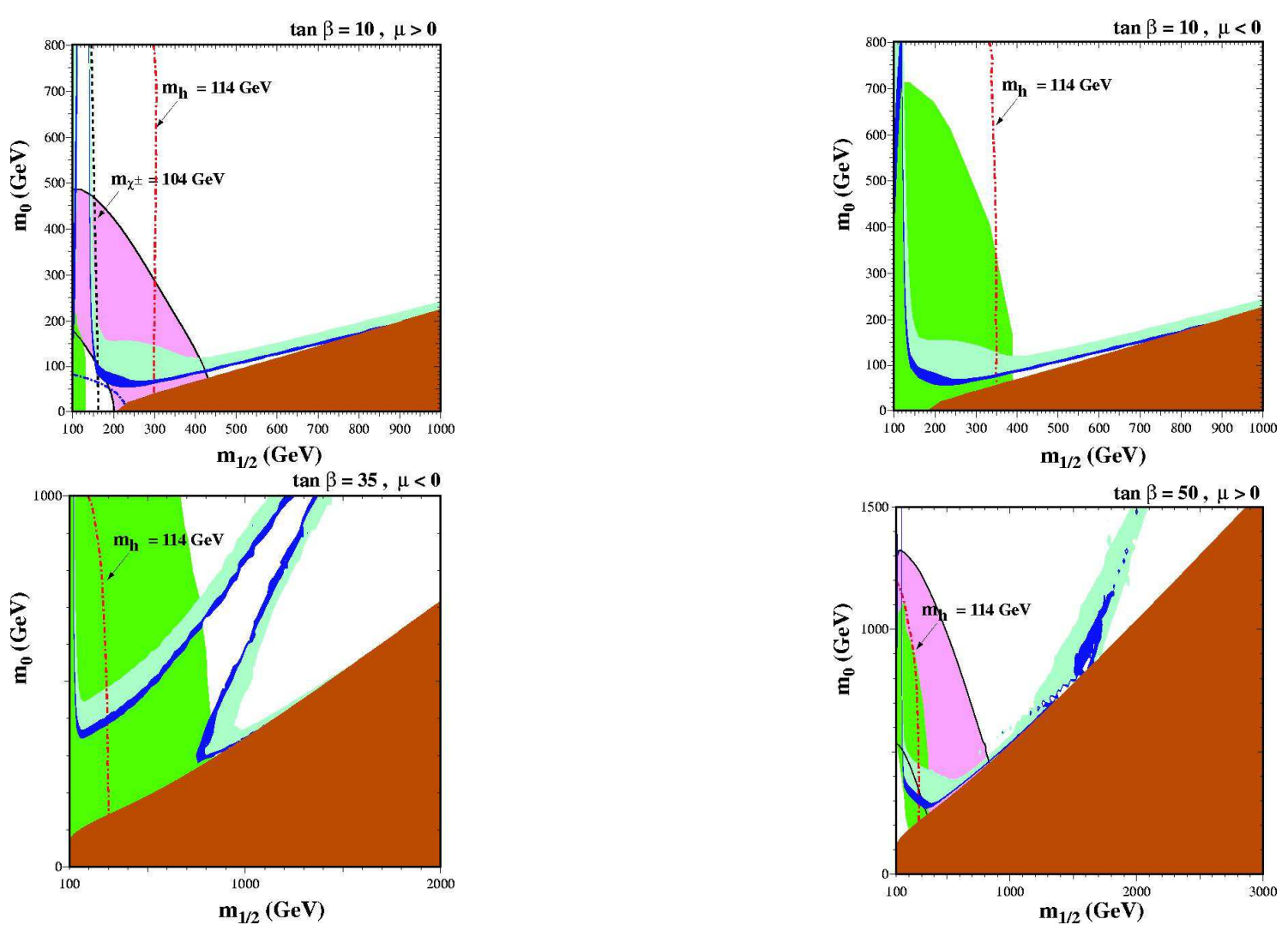

Figure 20: mSUGRA/CMSSM constraints after WMAP [68]. Dark Blue shaded region favoured by WMAP $\left(0.094 \leq \Omega_{\chi} h^{2} \leq 0.129\right)$. Turquoise shaded regions have $0.1 \leq$ $\Omega_{\chi} h^{2} \leq 0.3$. Brick red shaded regions are excluded because LSP is charged. Dark green regions are excluded by $b \rightarrow s \gamma$. The Pink shaded region includes $2-\sigma$ effects of $g_{\mu}-2$. Finally, the dash-dotted line represents the LEP constraint on ẽ mass. 

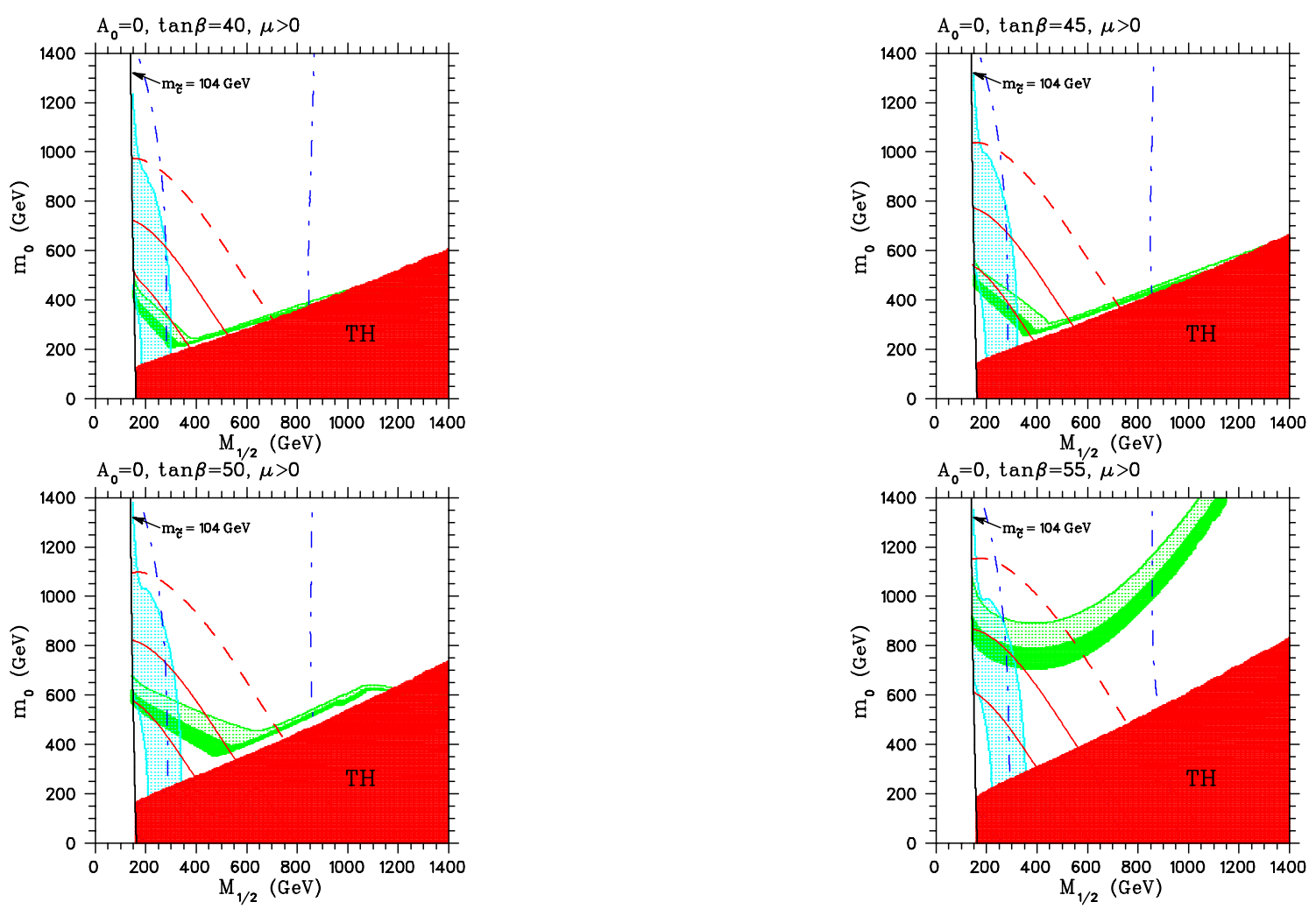

Figure 21: Cosmologically allowed regions of the relic density for in the $\left(m_{1 / 2}, m_{0}\right)$ plane for large tan $\beta$ values [69]. The mass of the top is taken $175 \mathrm{GeV}$. In the dark green shaded area $0.094<\Omega_{\chi_{1}^{0}} h_{0}^{2}<0.129$. In the light green shaded area $0.129<\Omega_{\chi_{1}^{0}} h_{0}^{2}<0.180$. The solid red lines mark the region within which the supersymmetric contribution to the anomalous magnetic moment of the muon is $\alpha_{\mu}^{S U S Y}=(361 \pm 106) \times 10^{-11}$. The dashed red line is the boundary of the region for which the lower bound is moved to its $2 \sigma$ limit. The dashed-dotted blue lines are the boundaries of the region $113.5 \mathrm{GeV} \leq m_{\text {Higgs }} \leq 117.0 \mathrm{GeV}$. The cyan shaded region is excluded due to $b \rightarrow s \gamma$ constraint. 
In figure 21 we see that for the $\tan \beta=55$ case, close to the highest possible value, and considering the $2 \sigma$ lower bound on the muon's anomalous magnetic moment $\alpha_{\mu}^{S U S Y} \geq$ $149 \times 10^{-11}$ and values of $\Omega_{\chi_{1}^{0}} h_{0}^{2}$ in the range $0.1126_{-0.0181}^{+0.0161}$, allowed points are within a narrow stripe. The point with the highest value for $m_{0}$ is (in $\left.\mathrm{GeV}\right)$ at $\left(m_{0}, m_{1 / 2}\right)=$ $(850,550)$ and that with the highest $M_{1 / 2}$ at $\left(m_{0}, m_{1 / 2}\right)=(750,600)$. The latter marks the lower end of the line segment of the boundary $149<10^{-11} \alpha_{\mu}^{S U S Y}$ which amputates the cosmologically allowed stripe.

It should be noted that within $1 \sigma$ of the E821 data only a few points survive which lie in a small region centered at $\left(m_{0}, m_{1 / 2}\right)=(725,300)$. The bounds on $m_{0}, m_{1 / 2}$ displayed in (the lower part of) figure 21 refer to the $A_{0}=0$ case. Allowing for $A_{0} \neq 0$ values, the upper bounds put on $m_{0}, M_{1 / 2}$ increase a little and so do the corresponding bounds on sparticle masses. For the LSP, the lightest of the charginos, stops, staus and Higgses the upper bounds on their masses are displayed in Table 1 for various values of the parameter $\tan \beta$, if the new WMAP determination [3, 5] of the Cold Dark Matter (13) and the $2 \sigma$ bound $149<10^{-11} \alpha_{\mu}^{S U S Y}<573$ of E821 is respected. We have also taken into account the limits arising from Higgs boson searches as well as from $b \rightarrow s \gamma$ experimental constraints. In extracting these values we used [69] a random sample of 40,000 points in the region $\left|A_{0}\right|<1 \mathrm{TeV}, \tan \beta<55, m_{0}, M_{1 / 2}<1.5 \mathrm{TeV}$ and $\mu>0$. The lightest of the charginos has a mass whose upper bound is $\approx 550 \mathrm{GeV}$, and this is smaller than the upper bounds put on the masses of the lightest of the other charged sparticles, namely the stau and stop, as is evident from Table 1 . Hence the prospects of discovering CMSSM at a $e^{+} e^{-}$collider with center of mass energy $\sqrt{s}=800 \mathrm{GeV}$, are not guaranteed. Thus a center of mass energy of at least $\sqrt{s} \approx 1.1 \mathrm{TeV}$ is required to discover SUSY through chargino pair production. Note that in the allowed regions the next to the lightest neutralino, $\tilde{\chi}^{\prime}$, has a mass very close to the lightest of the charginos and hence the process $e^{+} e^{-} \rightarrow \tilde{\chi} \tilde{\chi}^{\prime}$, with $\tilde{\chi}^{\prime}$ subsequently decaying to $\tilde{\chi}+l^{+} l^{-}$or $\tilde{\chi}+2$ jets, is kinematically allowed for such large $\tan \beta$, provided the energy is increased to at least $\sqrt{s}=860 \mathrm{GeV}$. It should be noted however that this channel proceeds via the $t$-channel exchange of a selectron and it is suppressed due to the heaviness of the exchanged sfermion. Therefore only if the center of mass energy is increased to $\sqrt{s}=1.1 \mathrm{TeV}$ supersymmetry can be discovered in a $e^{+} e^{-}$ collider provided it is based on the Constrained scenario [69].

An important conclusion, therefore, which can be inferred by inspecting the figures 20] 21] is that the constraints implied by a possible discrepancy of $g_{\mu}-2$ from the SM value $\left(\alpha_{\mu}^{S U S Y} \gtrsim 15.0 \times 10^{-10}\right)$, when combined with the WMAP restrictions on CDM (neutralino) relic densities (13), imply severe restrictions on the available parameter space of the EB and lower significantly the upper bounds on the allowed neutralino masses $m_{\chi_{1}^{0}}$. This is clearly seen in figure 22. In this the width of the strips is much smaller than the spacing between them. For $\tan \beta<40$ one obtains 68] $m_{\chi_{1}^{0}} \lesssim 500 \mathrm{GeV}$ (see figure 23), which is significantly lower than the bounds obtained in pre-WMAP cosmological models, 


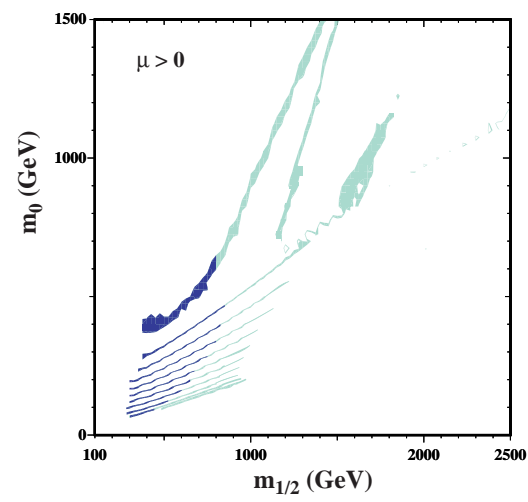

Figure 22: Strips correspond to tan $\beta=5,10,15,20,25,30,35,40,45,50,55$. Light Green Regions of the graph $\left(m_{0}, m_{1 / 2}\right)$ are those compatible with the WMAP result on the neutralino relic density $0.094 \leq \Omega_{\chi} h^{2} \leq 0.129$. Darker Blue shaded parts of strips are compatible with $g_{\mu}-2$ results (at a $2-\sigma$ level) [68].

$m_{\chi_{1}^{0}} \lesssim 650 \mathrm{GeV}$.

\begin{tabular}{|c|c|c|c|c|c|}
\hline \hline $\tan \beta$ & $\chi_{1}^{0}$ & $\chi_{1}^{+}$ & $\tilde{\tau}$ & $\tilde{t}$ & $h$ \\
\hline 10 & 155 & 280 & 170 & 580 & 116 \\
15 & 168 & 300 & 185 & 640 & 116 \\
20 & 220 & 400 & 236 & 812 & 118 \\
30 & 260 & 470 & 280 & 990 & 118 \\
40 & 290 & 520 & 310 & 1080 & 119 \\
50 & 305 & 553 & 355 & 1120 & 119 \\
55 & 250 & 450 & 585 & 970 & 117 \\
\hline \hline
\end{tabular}

Table 1: Upper bounds, in GeV, on the masses of the lightest of the neutralinos, charginos, staus, stops and Higgs bosons for various values of $\tan \beta$ if the new WMAP value [3] for $\Omega_{C D M} h^{2}$ and the $2 \sigma$ E821 bound, $149 \times 10^{-11}<\alpha_{\mu}^{S U S Y}<573 \times 10^{-11}$, is imposed [69].

To summarize, therefore, in the EB the recent WMAP data [3] can easily be adapted through by most of the benchmark points (in bulk and co-annihilation regions) of figure 19] by reducing $m_{0}$. In the post-WMAP benchmark scenaria 81] the only exception is the point $\mathrm{H}$ (tip of co-annihilation tail): WMAP data will lower $m_{1 / 2}$ thereby facilitating detection prospects of SUSY at LHC or future linear $e^{+} e^{-}$colliders a center of mass energy $\sqrt{s}=1.1 \mathrm{TeV}$ [69, 68], provided the HB region, and in particular its high zone (inversion), is not realized in nature. 

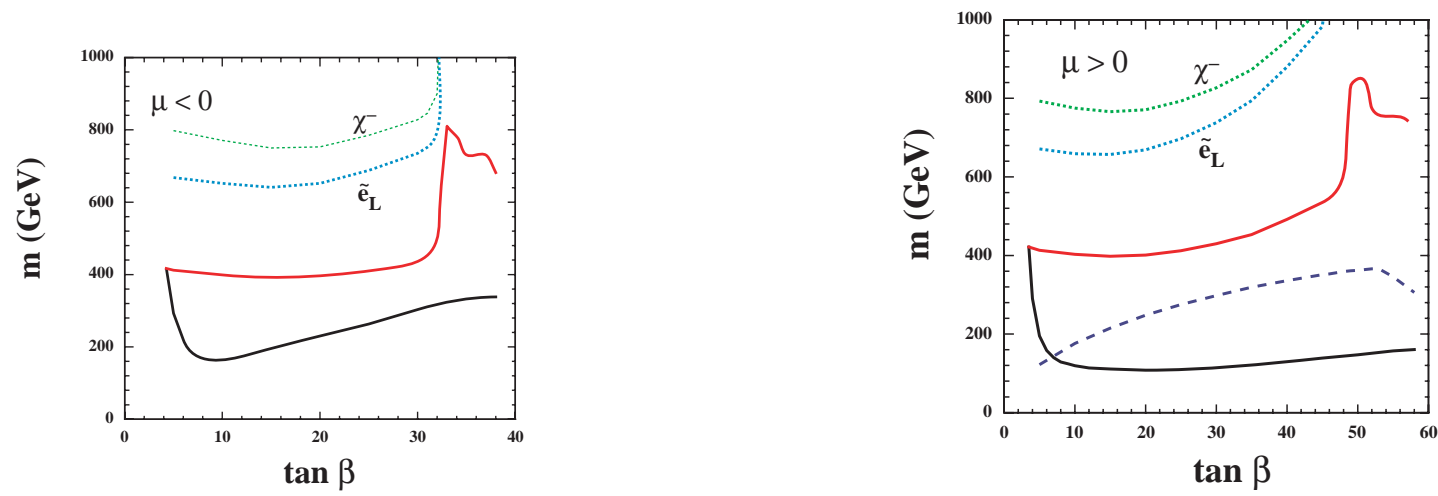

Figure 23: The Neutralino Mass $m_{\chi}$ Range in the EB of radiative symmetry breaking parameter space, implied by the WMAP data and other constraints $\left(b \rightarrow s \gamma, g_{\mu}-2\right.$ etc), in the context of the mSUGRA model [68]. Left: $\mu<0$, Right: $\mu>0$. Red solid lines: Upper limits without $g_{\mu}-2$. Dashed Blue lines: Upper limits with $g_{\mu}-2$. Dotted lines: Green: Masses of $\chi^{ \pm}$, Blue masses of $\tilde{e}_{L}$, at the tip of co-annihilation tails.

\subsubsection{Constraints in the $\mathrm{HB}$}

Despite the above-mentioned good prospects of discovering minimal SUSY models at future colliders, if the EB is realized, however, things may not be that simple in Nature. A recent $\chi^{2}$ study [41] of mSUGRA in light of the recent WMAP data has indicated that the $\mathrm{HB} /$ focus point region of the model's parameter space seems to be favoured along with the neutralino resonance annihilation region for $\mu>0$ and large $\tan \beta$ values. The favoured focus point region corresponds to moderate to large values of the Higgs parameter $\mu^{2}$, and large scalar masses $m_{0}$ in the several $\mathrm{TeV}$ range. The situation is summarized briefly in figure 24.

The situation in case the HB is included in the analysis is depicted in figure 25 33, where we plot the $m_{0}-m_{1 / 2}$ graphs, as well as graphs of $m_{0}, m_{1 / 2}$ vs the neutralino LSP mass. The neutralino density is that of the WMAP data. We stress again that, in case the high zone (inversion) region of the HB is realized, then the detection prospects of SUSY at LHC are diminished significantly, in view of the fact that in such regions slepton masses may lie in the several $\mathrm{TeV}$ range (see figure 25).

In view of the above results, an updated reach of LHC in view of the recent WMAP and other constraints discussed above (see figure 26) has been performed in [82, showing that a major part of the HB, but certainly not its high zone, can be accessible at LHC. The conclusion from this study 82 is that for an integrated luminosity of $100 \mathrm{fb}^{-1}$ values of $m_{1 / 2} \sim 1400 \mathrm{GeV}$ can be probed for small scalar masses $m_{0}$, corresponding to gluino 

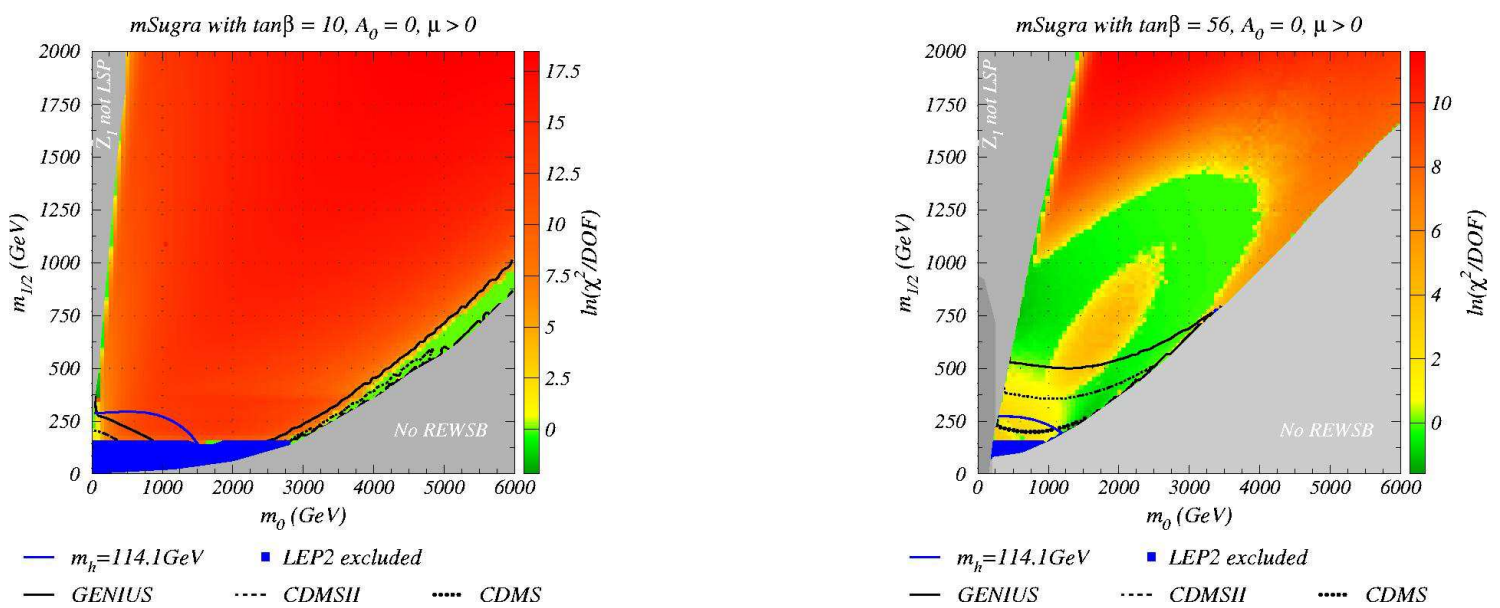

Figure 24: WMAP data seem to favour $\left(\frac{\chi^{2}}{d o f}<4 / 3\right)$ (green) the HB/focus point region (moderate to large values of $\mu$, large $m_{0}$ scalar masses) for almost all tan $\beta$ (Left), as well as $s$ - channel Higgs resonance annihilation (Right) for $\mu>0$ and large tan $\beta$ 41].

masses $m_{\tilde{g}} \sim 3 \mathrm{TeV}$. For large $m_{0}$, in the hyperbolic branch/focus point region, $m_{1 / 2} \sim 700$ $\mathrm{GeV}$ can be probed, corresponding to $m_{\tilde{g}} \sim 1800 \mathrm{GeV}$. It is also concluded that the LHC (CERN) can probe the entire stau co-annihilation region and most of the heavy Higgs annihilation funnel allowed by WMAP data, except for some range of $m_{0}, m_{1 / 2}$ in the case $\tan \beta \gtrsim 50$.

A similar updated reach study in light of the new WMAP data has also been done for the Tevatron [83], extending previous analyses to large $m_{0}$ masses up to $3.5 \mathrm{TeV}$, in order to probe the HB/focus region favoured by the WMAP data 41. Such a study indicated that for a $5 \sigma(3 \sigma)$ signal with $10(25) \mathrm{fb}^{-1}$ of integrated luminosity, the Tevatron reach in the trilepton channel extends up to $m_{1 / 2} \sim 190$ (270) $\mathrm{GeV}$ independent of $\tan \beta$, which corresponds to a reach in terms of gluino mass of $m_{\mathrm{g}} \sim 575(750) \mathrm{GeV}$.

\subsection{WMAP and Direct Dark Matter Searches}

We turn now to study the impact of the new WMAP data on Dark Matter, the constraints from $\left(g_{\mu}-2\right)$ and $b \rightarrow s \gamma$, and the Higgs mass bounds on direct Dark Matter searches. A quantity of great interest is the spin-independent ( scalar ) neutralino-proton cross section on which experimental limits exist from the current dark matter experiments $\sigma_{\chi^{0} p}(S I)<$ $10^{-6}$ picobarns.

In figure 27] we plot the scalar $\chi_{1}^{0}$-nucleon cross section as function of the LSP mass, $m_{\chi_{1}^{0}}$ 69. On the top of it the shaded region (in cyan colour) is excluded by the CDMS experiment 84]. The DAMA sensitivity region is plotted in yellow 85]. Pluses $(+)$ (in blue 


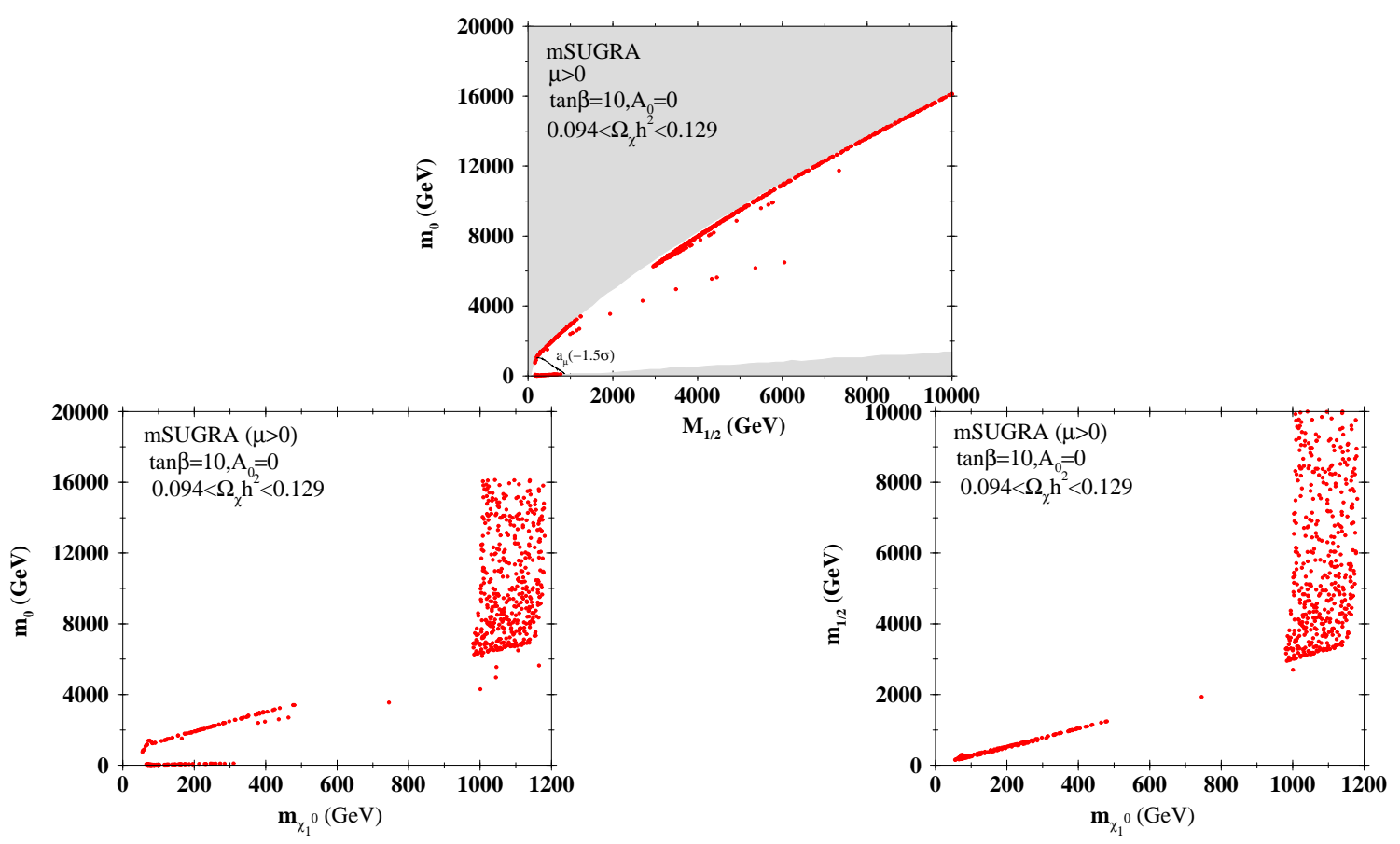

Figure 25: $m_{0}-m_{1 / 2}$ graph, and $m_{0}$ and $m_{1 / 2}$ vs. $m_{\chi}$ graphs, including the $H B$ of mSUGRA [33]. Such regions are favoured by the WMAP data [41].

colour) represent points which are both compatible with the E821 data $\alpha_{\mu}^{\mathrm{SUSY}}=(361 \pm$ $106) \times 10^{-11}$ and the WMAP cosmological bounds $\Omega_{C D M} h^{2}=0.1126_{-0.0181}^{+0.0161}$. Diamonds $(\diamond)$ (in green colour) represent points which are compatible with the $2 \sigma$ E821 data $149 \times$ $10^{-11}<\alpha_{\mu}^{\mathrm{SUSY}}<573 \times 10^{-11}$ and the cosmological bounds. The crosses $(\times)$ (in red colour) represent the rest of the points of our random sample which consists of 40,000 points in the range $\left|A_{0}\right|<1 \mathrm{TeV}, m_{0}, m_{1 / 2}<1.5 \mathrm{TeV}, \tan \beta<55$ and $\mu>0$ belonging to the EB region. Here the Higgs boson mass, $m_{h}>113.5 \mathrm{GeV}$ and the $b \rightarrow s \gamma$ bounds have been properly taken into account [69]. From this figure it is seen that the points which are compatible both with the $\left(g_{\mu}-2\right)$ E821 and the cosmological data can yield cross sections slightly above $10^{-8} \mathrm{pb}$ when $m_{\chi_{1}^{0}}$ is about $120 \mathrm{GeV}$. The maximum value of $m_{\chi_{1}^{0}}$ is around $200 \mathrm{GeV}$ but in this case the scalar cross section drops by almost an order of magnitude $10^{-9} \mathrm{pb}$. Accepting the $2 \sigma\left(g_{\mu}-2\right)$ bound the maximum value of the scalar cross section is again $\approx 10^{-8} \mathrm{pb}$, for $m_{\chi_{1}^{0}} \approx 120 \mathrm{GeV}$, but the $m_{\chi_{1}^{0}}$ bound is increased to about $280 \mathrm{GeV}$ at the expense of having cross sections slightly smaller than $10^{-9} \mathrm{pb}$. Considering the $\mu>0$ case, it is very important that using all available data 69, one can put a lower bound $\approx 10^{-9}$ pbarns on the scalar cross section which is very encouraging for 

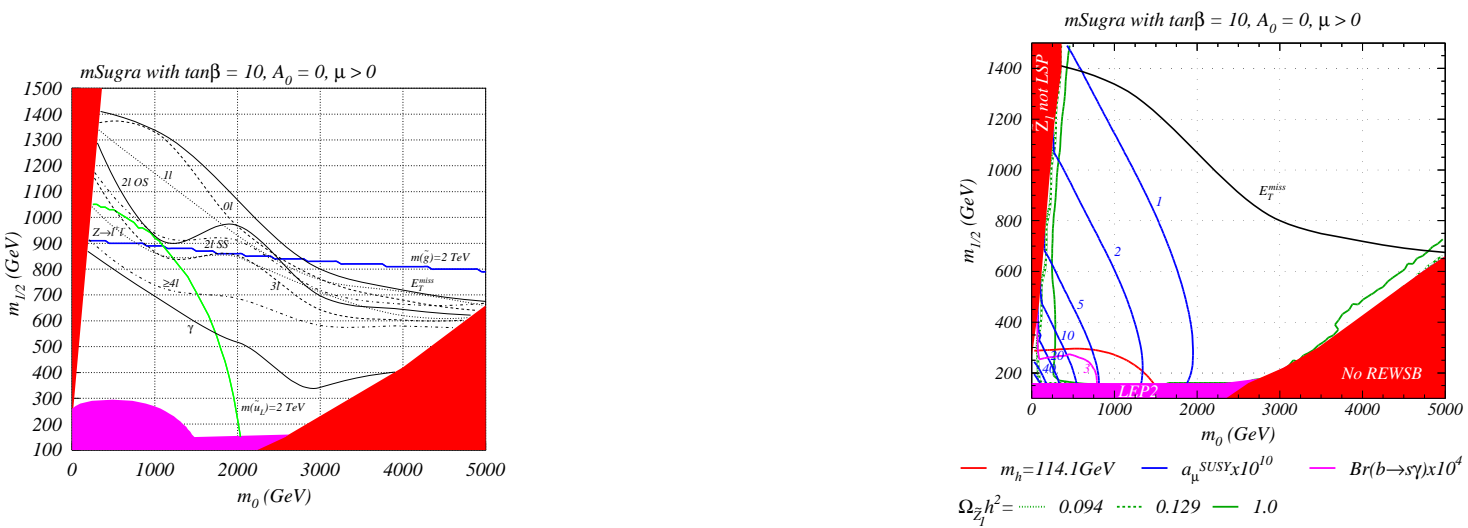

Figure 26: Left: The updated Reach in $\left(m_{0}, m_{1 / 2}\right)$ parameter plane of mSUGRA assuming $100 \mathrm{fb}^{-1}$ integrated luminosity. Red (magenta) regions are excluded by theoretical (experimental) constraints [82]. Right: Contours (in view of the uncertainties) of several

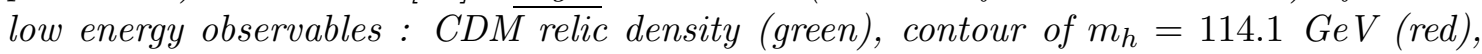
contours of $a_{\mu} 10^{10}$ (blue) and contours of $b \rightarrow s \gamma B F\left(\times 10^{4}\right)$ (magenta).

future DM direct detection experiments [86]. Such a lower bound cannot be imposed when $\mu<0$, since the scalar cross section can become very small due to accidental cancellations between the sfermion and Higgs exchange processes. However, this case is not favoured by $\left(g_{\mu}-2\right)$ and $b \rightarrow s \gamma$ data.

Portions of the high zone (inversion) region of the HB may be detected by such direct Dark Matter Search Experiments, which are thus complementary to collider physics. As in the EB case such scattered plots can be produced also for the HB [33, which seems to be favoured by the WMAP data [41, and which has regions that lie beyond the reach of LHC. As stressed in [33] it is important to distinguish the spin-dependent from the spin-independent (scalar) cross sections in this branch.

In figure 28 we give a plot of the scalar cross section for $\tan \beta=10$ and $\mu>0$. We do observe that the lower rapidly falling curve that terminates at $m_{\chi}^{0}=300 \mathrm{GeV}$ is the branch on which staus co-annihilation occurs. The upper curve arises from the low zone of the HB, while the patch to the right is the one corresponding to the inversion region of the HB [33]. This patch indicates that the scalar cross sections are quite significant even though one is in the inversion region in the range $10^{-10}-10^{-7}$ pbarns. Thus, despite the fact that the direct detection of SUSY in the inversion region is difficult, the neutralino-proton scalar cross section are still substantial. The sensitivity of the future dark matter detectors 86 will reach $10^{-9}$ pbarns, being capable of probing a significant portion of the parameter space of fig. 28, In this latter respect we also mention novel indirect detectors of SUSY dark matter [87], exploiting possible detector of cosmic antimatter (antideuteron), as a 


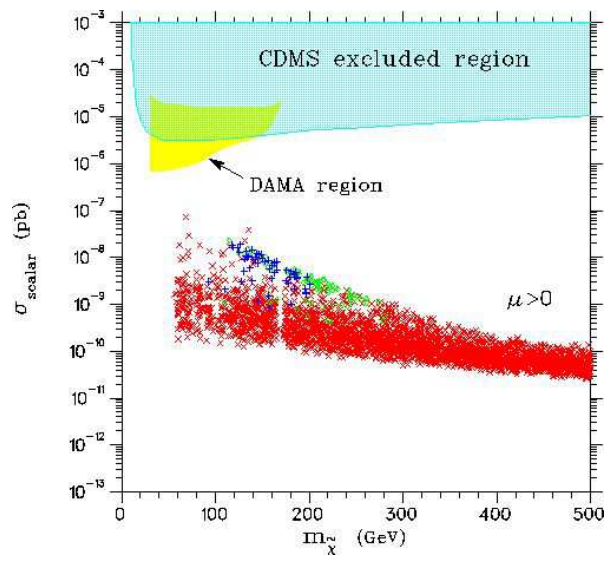

Figure 27: Neutralino-nucleon scalar cross section vs. $m_{\chi}$ in the EB [69].

result of neutralino interactions (see figure 29), which could detect neutralinos in a mass range from 80-350 GeV, much higher than other future dark matter experiments [86].

Finally, in figure 30 we plot the spin dependent parts of the neutralino-proton cross section, for the same range of parameters as in figure 28. A comparison between these two figures shows that the spin dependent cross section is much larger, by three or four orders of magnitude, than the spin independent one. One can repeat the analysis to find that for $\tan \beta \leq 50$ the neutralino mass range consistent with the WMAP constraints on the branch corresponding to neutralino-stau coannihilation is $m_{\chi_{1}^{0}} \leq 500 \mathrm{GeV}$ 68, 69, and $m_{\chi_{1}^{0}} \leq 1200 \mathrm{GeV}$ for the high zone of the $\mathrm{HB}$, where the relic density constraints are satisfied due to coannihilation with the next to lightest neutralino and light chargino 33, as discussed previously. We repeat again that these constraints remain intact under the imposition of the Estimate (I) of the $g_{\mu}-2$, while the constraint arising from the inversion region of the HB is removed under the imposition of the Estimate (II) of the $g_{\mu}-2$. We reiterate, therefore, once more the importance of having unambiguous measurements of this quantity in the immediate future, which will be indispensable in guiding supersymmetric searches in future colliders.

\subsection{WMAP, Proton Decay and SUSY Constraints in Grand Unified Supersymmetric Models}

In view of the above-described constraints on MSSM embedded in mSUGRA, it is also natural to ask whether additional constraints are implied by the lower limit on the proton life time which is a prediction of Grand Unified Theories (GUTs). So far we did not consider grand unification in the above analysis.

There are a number of important issues associated with such an extension. One is 


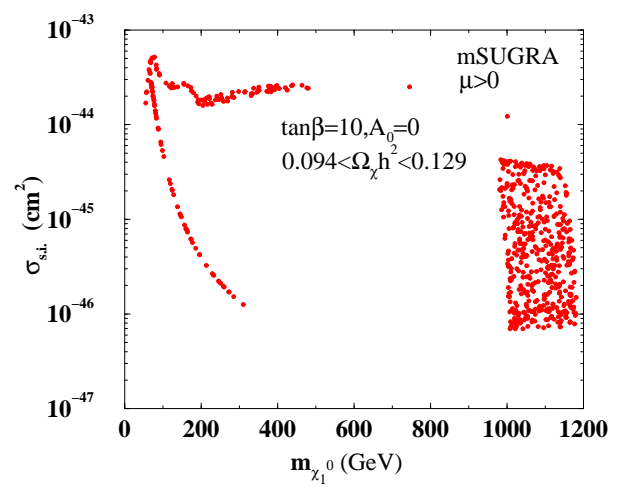

Figure 28: Spin independent part of the neutralino-proton cross section vs. $m_{\chi^{0}}$ including the $H B$ [33].

the exact value of $\sin ^{2} \theta_{W}$, which acquires important corrections from threshold effects at the electroweak scale, associated with the spectrum of MSSM particles [88, 89], and at the grand unification scale, associated with the spectrum of GUT supermultiplets [88, 90]. Precision measurements indicate a small deviation of $\sin ^{2} \theta_{W}$ even from the value predicted in a minimal supersymmetric $S U(5)$ GUT, assuming the range of $\alpha_{s}\left(M_{Z}\right)$ now indicated by experiment 91 .

The second issue is the lifetime of the proton. Minimal supersymmetric $S U(5)$ avoids the catastrophically rapid $p \rightarrow e^{+} \pi^{0}$ decay that scuppered non-supersymmetric $S U(5)$. However, supersymmetric $S U(5)$ predicts $p \rightarrow \bar{\nu} K^{+}$decay through $d=5$ operators 92,93 at a rate that may be too fast 94 to satisfy the presently available lower limit on the lifetime for this decay [95, 96]. The latter requires the $S U(5)$ colour-triplet Higgs particles to weigh $>7.6 \times 10^{16} \mathrm{GeV}$, whereas conventional $S U(5)$ unification for $\alpha_{s}\left(M_{Z}\right)=0.1185 \pm$ $0.002, \sin ^{2} \theta_{W}=0.23117 \pm 0.00016$ and $\alpha_{e m}\left(M_{Z}\right)=1 /(127.943 \pm 0.027)$ 96] would impose the upper limit of $3.6 \times 10^{15} \mathrm{GeV}$ at the $90 \%$ confidence level 94. This problem becomes particularly acute if the sparticle spectrum is relatively light, as would be indicated if the present experimental and theoretical central values of $\left(g_{\mu}-2\right)$ [66] remain unchanged as the errors are reduced.

The simplest way to avoid these potential pitfalls is to flip $S U(5)$ 97, 98. As is well known, flipped $S U(5)$ offers the possibility of decoupling somewhat the scales at which the Standard Model $S U(3), S U(2)$ and $U(1)$ factors are unified. This would allow the strength of the $U(1)$ gauge to become smaller than in minimal supersymmetric $S U(5)$, for the same value of $\alpha_{s}\left(M_{Z}\right)$ [89]. Moreover, in addition to having a longer $p \rightarrow e / \mu^{+} \pi^{0}$ lifetime than non-supersymmetric $S U(5)$, flipped $S U(5)$ also suppresses the $d=5$ operators that are dangerous in minimal supersymmetric $S U(5)$, by virtue of its economical missing-partner mechanism [97. 


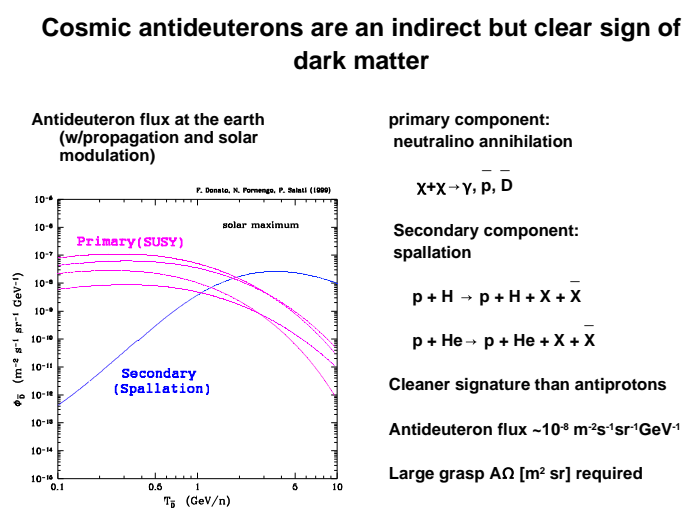

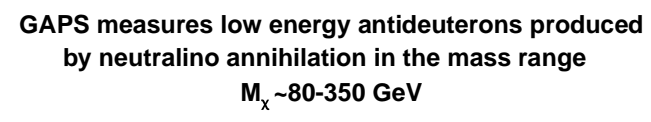
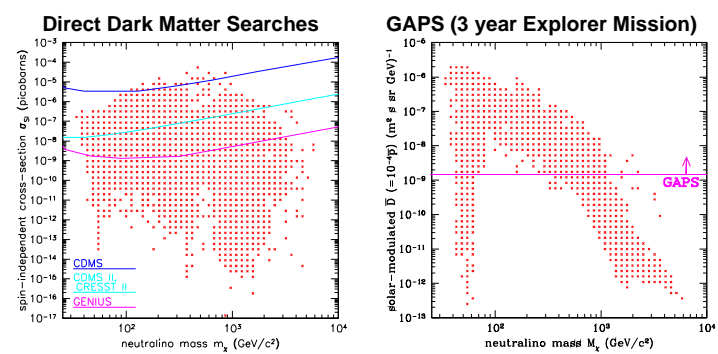

Figure 29: GAPS Detector of Indirect SUSY Dark Matter Searches, through the detection of cosmic antideuteron, may cover a neutralino mass range that may be complementary to all other direct dark matter and collider searches [87].

In 99, the authors re-analyzed the issues of $\sin ^{2} \theta_{W}$ and proton decay in flipped $S U(5)$ [89], in view of the most recent precise measurements of $\alpha_{s}\left(M_{Z}\right)$ and $\sin ^{2} \theta_{W}$, and the latest (at the time of publication) limits on supersymmetric particles (before publication of WMAP data). The analysis of such issues was done in the framework of CMSSM, as above, and consisted of both a general analysis in the $\left(m_{1 / 2}, m_{0}\right)$ plane and also more detailed specific analyses of benchmark CMSSM parameter choices that respected all the available experimental constraints at the time 65. It was found that the $p \rightarrow e / \mu^{+} \pi^{0}$ decay lifetime exceeds the present experimental lower limit 95], with a significant likelihood that it may be accessible to the next round of experiments [100]. In the context of the present article we shall not present the analysis in great detail, in particular we shall not discuss the ambiguities and characteristic ratios of proton decay modes in flipped $S U(5)$. We refer the reader to the existing literature for this [99]. Instead we shall give a comprehensive description of their results in figures 31] 32, which are self explanatory.

In figure 31 we give the pre-WMAP results of [99], while in figure 32] we give the updated post WMAP figures (taking into account the updated benchmark points [81]), for comparison. From figs. 31] and 32 it is evident that the 'bulk' regions of the parameter space preferred by astrophysics and cosmology, which occur at relatively small values of $\left(m_{1 / 2}, m_{0}\right)$, generally correspond to $\tau\left(p \rightarrow e^{+} \pi^{0}\right) \sim(1-2) \times 10^{35}$ y. However, these 'bulk' regions are generally disfavoured by the experimental lower limit on the Higgs mass, $m_{h}$, and/or by $b \rightarrow s \gamma$ decay. Larger values of $\tau\left(p \rightarrow e^{+} \pi^{0}\right)$ are found in the 'tail' regions of the cosmological parameter space, which occur at large $m_{1 / 2}$ where $\chi-\tilde{\ell}$ coannihilation may be important, and at larger $m_{1 / 2}$ and $m_{0}$ where resonant direct-channel annihilation 


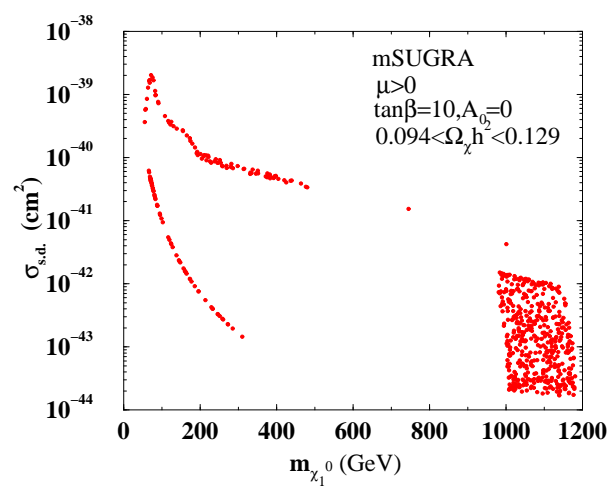

Figure 30: Spin dependent part of the neutralino-proton cross section vs. $m_{\chi^{0}}$ including the $H B$ [33].

via the heavier Higgs bosons $A, H$ may be important.

Another issue studied in 99] were the possible implications of the GUT threshold effect $\delta_{\text {heavy }}$ [88, 90]. A general expression for this in flipped $S U(5)$ is given in 88, where we refer the interested reader. In fig. 33 we display 99 the possible numerical effects of $\delta_{\text {heavy }}$ on $\tau\left(p \rightarrow e / \mu^{+} \pi^{0}\right)$ in the various benchmark scenarios, assuming the plausible ranges $-0.0016<\delta_{\text {heavy }}<0.0005$ [89]. The boundary between the different shadings for each strip corresponds to the case where $\delta_{\text {heavy }}=0$. The left (red) parts of the strips show how much $\tau\left(p \rightarrow e^{+} \pi^{0}\right)$ could be reduced by a judicious choice of $\delta_{\text {heavy, }}$ and the right (blue) parts of the strips show how much $\tau\left(p \rightarrow e^{+} \pi^{0}\right)$ could be increased. The inner bars correspond to the uncertainty in $\sin ^{2} \theta_{W}$. On the optimistic side, we see that some models could yield $\tau\left(p \rightarrow e^{+} \pi^{0}\right)<10^{35} \mathrm{y}$, and all models might have $\tau\left(p \rightarrow e^{+} \pi^{0}\right)<5 \times 10^{35}$ y. However, on the pessimistic side, in no model can we exclude the possibility that $\tau\left(p \rightarrow e^{+} \pi^{0}\right)>10^{36} \mathrm{y}$.

We notice at this stage that a new generation of massive water-Čerenkov detectors weighing up to $10^{6}$ tonnes is being proposed [100], that may be sensitive to $\tau\left(p \rightarrow e^{+} \pi^{0}\right)<$ $10^{35} \mathrm{y}$. According to the calculations of [99], such an experiment has a chance of detecting proton decay in flipped $S U(5)$, though nothing can of course be guaranteed. We also mention [103, 104] that flipped $S U(5)$ makes predictions for ratios of decay rates involving strange particles, neutrinos and charged leptons that differ characteristically from those of conventional $S U(5)$. Comparing the rates for $e^{+}, \mu^{+}$and neutrino modes would give novel insights into GUTs as well as mixing patterns.

We conclude from the above analyses 99, therefore, that flipped $S U(5)$ evades two of the pitfalls of conventional supersymmetric $S U(5)$ : (i) it offers the possibility of lowering the prediction for $\alpha_{s}\left(M_{Z}\right)$ for any given value of $\sin ^{2} \theta_{W}$ and choice of sparticle spectrum, and (ii), as far as proton decay is concerned, flipped $S U(5)$ suppresses $p \rightarrow \bar{\nu} K^{+}$ 

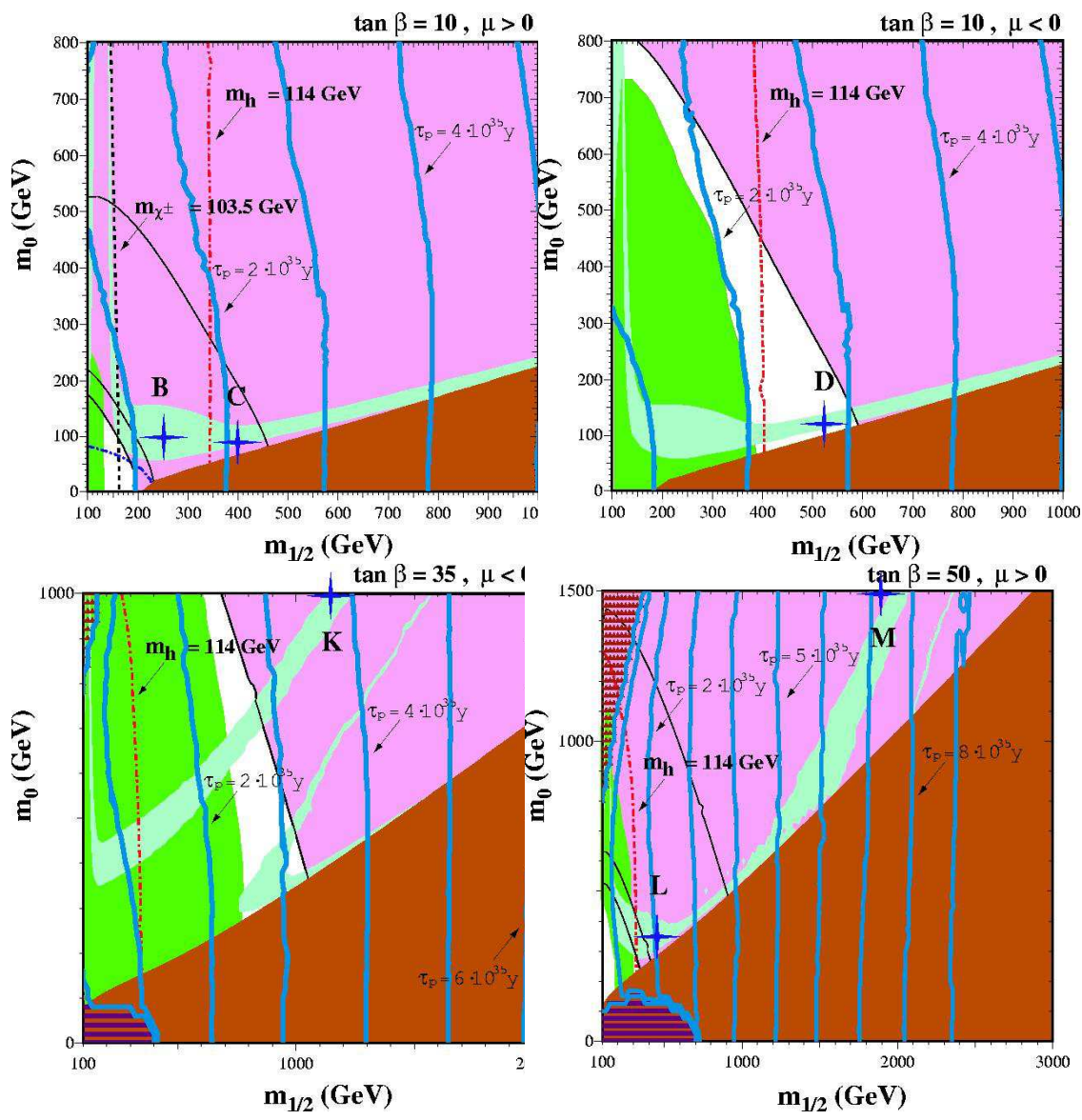

Figure 31: Pre-WMAP constraints on supersymmetric flipped SU(5) model [99]. The solid (blue) lines are contours of $\tau\left(p \rightarrow e / \mu^{+} \pi^{0}\right)$ in the $\left(m_{1 / 2}, m_{0}\right)$ plane for the CMSSM with (a) $\tan \beta=$ $10, \mu>0$, (b) $\tan \beta=10, \mu<0$, (c) $\tan \beta=35, \mu<0$ and (d) $\tan \beta=50, \mu>0$. The (blue) crosses indicate the CMSSM benchmark points with the corresponding value of $\tan \beta$ and sign of $\mu$ [65]. Following [101], the dark (red) shaded regions are excluded because the LSP is charged, the light (turquoise) shaded regions have $0.1<\Omega_{\chi} h^{2}<0.3$, intermediate (green) shaded regions at low $m_{1 / 2}$ are excluded by $b \rightarrow s \gamma$, shaded (pink) regions at large $\left(m_{1 / 2}, m_{0}\right)$ are consistent with $g_{\mu}-2$ at the 2- $\sigma$ level, and electroweak symmetry breaking is not possible in the hatched regions. The nearvertical dashed (black) lines correspond to the LEP lower limit on chargino mass, $m_{\chi}^{ \pm}=103.5 \mathrm{GeV}$, the dot-dashed (red) lines to the Higgs mass, $m_{h}=114 \mathrm{GeV}$ as calculated using the FeynHiggs code [102], and the dotted (blue) lines at small $\left(m_{1 / 2}, m_{0}\right)$ to $m_{\tilde{e}}=100 \mathrm{GeV}$. 

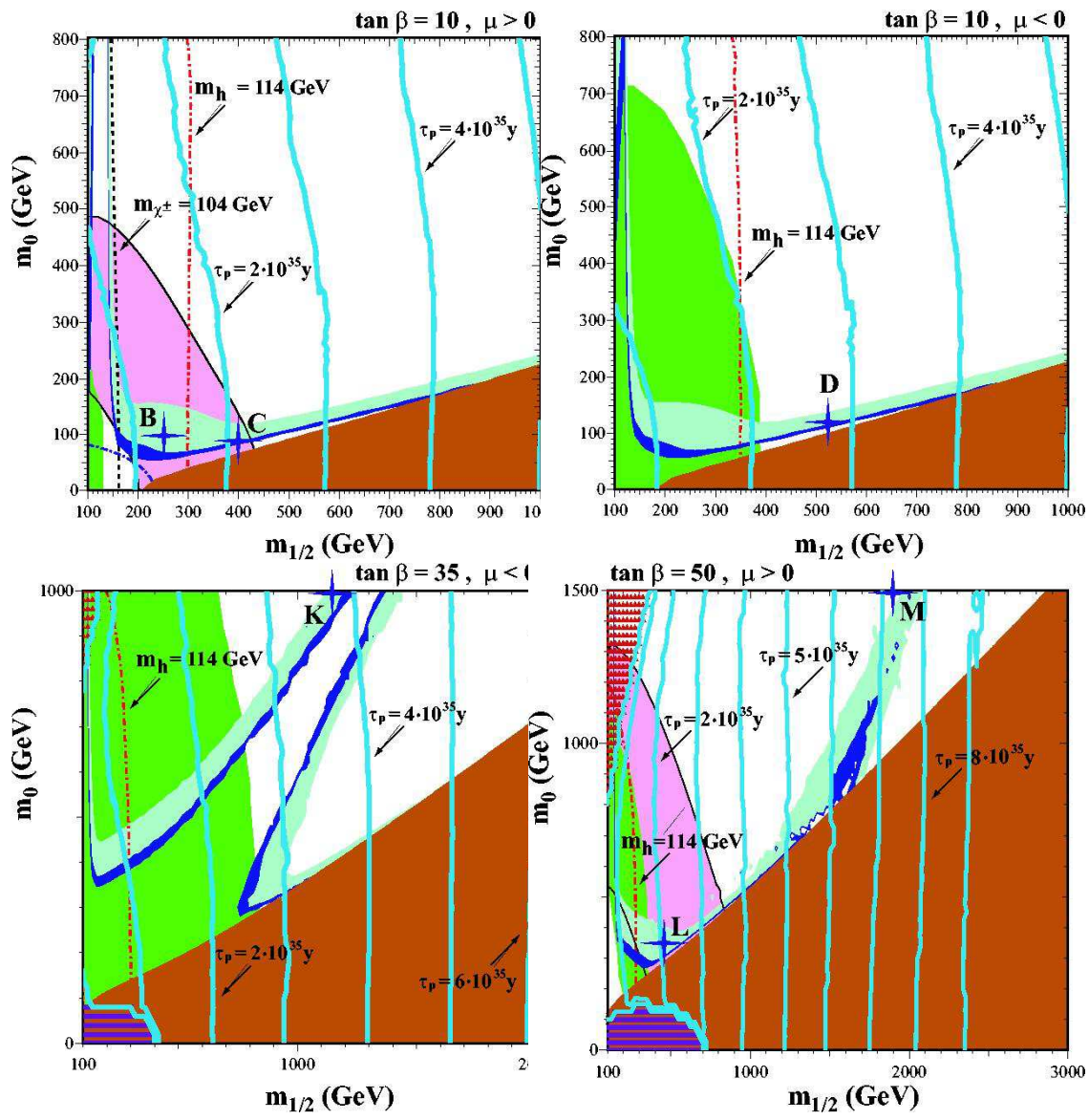

Figure 32: As in figure 31 but taking into account the new cosmological constraints from WMAP on relic densities (dark blue regions). The contours of $\tau\left(p \rightarrow e / \mu^{+} \pi^{0}\right)$ are given here by the light-blue solid lines (we are grateful to J. Walker for providing us with these updated figures). 


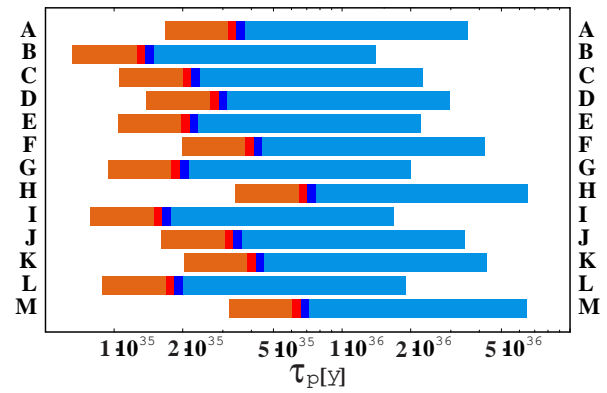

Figure 33: For each of the CMSSM benchmark points, this plot shows, by the lighter outer bars, the range of $\tau\left(p \rightarrow e / \mu^{+} \pi^{0}\right)$ attained by varying $\delta_{\text {heavy }}$ over the range -0.0016 to +0.0005 [89]. The central boundary of the narrow inner bars (red, blue) corresponds to the effect of $\delta_{\text {light }}$ alone, with $\delta_{\text {heavy }}=0$, while the narrow bars themselves represent uncertainty in $\sin ^{2} \theta_{W}$. We see that heavy threshold effects could make $\tau\left(p \rightarrow e / \mu^{+} \pi^{0}\right)$ slightly shorter or considerably longer.

decay naturally via its economical missing-partner mechanism. As in conventional supersymmetric $S U(5)$, the lifetime for $p \rightarrow e / \mu^{+} \pi^{0}$ decay generally exceeds the present experimental lower limit. However, as shown in [99], the flipped $S U(5)$ mechanism for reducing $\alpha_{s}\left(M_{Z}\right)$ reduces the scale at which colour $S U(3)$ and electroweak $S U(2)$ are unified, bringing $\tau\left(p \rightarrow e / \mu^{+} \pi^{0}\right)$ tantalizingly close to the prospective sensitivity of the next round of experiments. Proton decay has historically been an embarrassment for minimal $S U(5)$ GUTs, first in their non-supersymmetric guise and more recently in their minimal supersymmetric version. The answer may be to flip $S U(5)$ out of trouble. We remark also that flipped $S U(5)$ grand unified supersymmetric theories arise naturally in intersecting brane models [105].

\section{Dark Energy: Cosmological Constant or Quintessence or...?}

Before ending this review we would like to devote a few pages on an important matter, which so far has not been properly taken into account. In our constrained minimal models above, we have taken into consideration the WMAP results concerning dark matter density constraints, which constitutes only $23 \%$ of the Uninerse energy density content. However, as mentioned in section two, a plethora of recent data, including those of WMAP, has indicated that $73 \%$ of the Universe energy consists of an unknown substance, termed dark energy (c.f. figures [18).

The origin of this component is as yet unknown, despite enormous theoretical effort. 
In the simplest approach, the dark energy is nothing other but a cosmological constant. A negative cosmological constant was introduced by Einstein in the original theory of relativity to enforce compatibility of Einstein's equation of General Relativity with a static Universe, believed (wrongly) to be the case of the observed Universe at the time. Hubble's observation on the expansion of the Universe (1927) removed the necessity for introducing such a constant into the theory. Ever since, until five years ago, theoretical physicists believed that the vacuum energy density of the Universe was zero, and they were trying (alas in vain!) to find a symmetry reason for that. The situation changed drastically recently, after experimental evidence for a present-era acceleration of the Universe. A FRW Universe with a positive cosmological constant constitutes the best fit model of the WMAP and supernovae data [5, 1]. From a theoretical viewpoint, however, this poses a serious threat in quantization. Indeed, a de Sitter Universe, which would be the final state of such a positive cosmological constant FRW Universe, due to the expansion of the Universe which leads to a dilution of any matter density, is characterized by the presence of an event horizon, making the proper definition of asymptotic states, and hence a scattering matrix ( $S$-matrix), problematic. This presents serious challenges for a proper quantization of the theory, which are unresolved at present [106]. It also poses a serious challenge for string theory [107, 108], given that the latter, at least in its initial perturbative form, has been formulated as an S-matrix theory.

Perhaps a more satisfactory, from this point of view, approach to the problem of a physical explanation of the currently observed acceleration of the Universe [1, and the deduced dark energy component [1, [5] is to assume a time varying vacuum energy, $\Lambda(t)$ relaxing to zero asymptotically in the Robertson-Walker time [109, 110, $t \rightarrow \infty$.

One may obtain this time dependent "vacuum energy" by means of a (scalar) field, which has not relaxed yet to the minimum of its potential. Such a mechanism, known as quintessence, has attracted a lot of attention [111, and represents an elegant nonequilibrium approach to the issue of a positive cosmological constant. The important advantage of quintessence (or other relaxation) models is the possibility of defining asymptotic states, and hence an $S$-matrix, properly, due to the asymptotic vanishing of the vacuum energy, when the system reaches its equilibrium. Another important advantage is that there exist attractor solutions in a cosmological setting for some classes of potentials [112, which imply that in some cases the scalar field will join the attractor solution before the present epoch for a wide range of initial conditions, thereby evading (in principle) fine tuning and tracking problems. There are, however, serious issues that remain unresolved, most of them associated with the nature of the tracking quintessence field, which should be important today, but not in the past of our Universe. Another important issue is the current coincidence in order of magnitude of the dark energy density with the matter energy density of the Universe, which quintessence models are called to explain in a fine-tuning free way.

A natural relaxation mechanism, compatible with the current value of $\Lambda\left(t_{\text {present }}\right) \lesssim$ 
$10^{-120} M_{P}^{4}$ inferred from the observations, would be the one in which 109 110 $\Lambda(t) \sim$ $M_{P}^{4} / t^{2}$, for long times $M_{P} t \gg 1$, where $M_{P} \sim 10^{19} \mathrm{GeV}$ is the four-dimensional Planck energy scale. For $M_{P} t_{\text {present }} \gtrsim 10^{60}$, which is the Age of the (observed) Universe, one obtains naturally the above limits.

Such inverse square law relaxation models exist in the literature, in either field or string/brane quintessence theory [109, 110, or even in non-equilibrium approaches to string theory, such as non-critical Liouville strings [15, 17, 18, 19, where the vacuumenergy relaxation law is obtained from first principles, specifically, by means of world-sheet conformal field theory methods.

In this last respect the quintessence field is actually provided by the Liouville mode itself, which is viewed as the time variable. It is the nature of non-critical strings to associate the Liouville mode with a linear in time part of the dilaton, and hence noncritical string quintessence may actually be viewed as a dilaton quintessence, which also characterizes some other approaches within critical string theory 113. The advantage of dilaton quintessence is that the scalar dilaton field exist in the string spectrum, and there is no need to assume extra fundamental scalars, as is the case in quintessence models of local field theory.

There is also another aspect of non-critical strings, which makes them interesting, at least from a theoretical viewpoint. Unlike critical strings, which are formulated as a theory of target-space scattering amplitudes (S-matrix), Liouville strings do not necessarily have a well-defined target-space S-matrix [20, 108]. Upon the identification of target-time with the Liouville mode [20], the world-sheet Liouville correlators among vertex operators may admit the interpretation of non-factorizable $\$$-matrix elements, acting on density matrices rather than target-space state vectors ${ }^{4}, \rho_{\text {out }}=\$ \rho_{\text {in }}$, with $\$ \neq S S^{\dagger}$, with $S$ the ordinary S-matrix, which may thus not be well defined. However, we stress that the operator $\$$ is well defined in Liouville strings, as being associated with correlation functions of vertex operators [20].

Such non-critical string models are then capable of describing consistently even quantum cosmological models with a non-zero cosmological constant, which, as we mentioned earlier, are hamprered in a field theory context by the lack of an S-matrix formalism due to the existence of cosmic horizons. The lack of a proper $S$-matrix may also have implications on violations of CPT symmetry in such systems [114, 20], with interesting phenomenological consequences in both particle physics and cosmology [115]. Of course, in Liouville-quintessence relaxation string models one may be able of defining an S-matrix

\footnotetext{
${ }^{4}$ The presence of density matrices implies an analogy of Liouville strings with open quantum mechanical systems [20. The analogy is exact, given that in such models there are modes which are not accessible by low-energy scattering experiments, such as topological gravitational modes or back-reaction spacetime foam effects, or, in colliding brane world scenaria, recoil modes etc.. Intgegrating out such modes from a low-energy observer, defines an effective low-energy string theory, which is non critical and out of equilibrium. It is such theories that cosmological models are based upon in this context [15, 17, 18, 19.
} 
asymptotically in time $t$, given the possibility of defining properly asymptotic states at the equilibrium point $t \rightarrow \infty$, where critical string theory is reached in such cases.

As we have mentioned previously, in section 3, in some string theory models the dilaton has also been viewed as the inflaton [14, 22, 15, 17, 18, 19. It would be nice to find a consistent realistic model in which a single field can explain both phases of the Universe. From the point of view of the acceleration of the Universe, implied by the dark energy, such an assumption is not unnatural given that currently the Universe appears to enter an accelerating de Sitter phase which is qualitatively similar to that of (the beginning of) inflation. This may be the result of a particular dilaton potential in a string/brane model. However, this issue is still far from being resolved, given that it is associated with non perturbative effects in string theory which are not at hand.

From the phenomenological point of view quintessence models are still compatible with the current observations, including those of WMAP [116, 5]. Indeed, as discussed in section 2 (c.f. figure 9), the WMAP best fit model analysis has provided us with the following range of the values of the quintessence field $\mathcal{Q}$ equation of state:

$$
\begin{aligned}
& w_{\mathcal{Q}}=\frac{p_{\mathcal{Q}}}{\rho_{\mathcal{Q}}}=\frac{(\dot{\mathcal{Q}})^{2} / 2-V(\mathcal{Q})}{(\dot{\mathcal{Q}})^{2} / 2+V(\mathcal{Q})} \\
& -1 \leq w \leq-0.78
\end{aligned}
$$

where $V(\mathcal{Q})$ is the potential of the quintessence, and the lower limit is theoretical, corresponding to the cosmological constant model (if this theoretical assumption is relaxed the upper limit becomes slightly larger, $\sim-0.67[\underline{5}]$ ).

Such values appear naturally in many quintessence models [111, 17, 18, 19. From our point of view in this work we would like to concentrate and review approaches that embed such quintessence scenaria in supergravity models [117, 118, 119. We note that, in this conext, the first phenomenological approach was given in [110], well before the recent astrophysical data 3, 1. Such models could in principle be used, then, to constraint minimal supersymmetric models, such as MSSM, in the way mSUGRA was used above, but this time taking into account the dark energy component of the Universe energy density ${ }^{5}$.

The issue of looking at quintessence in supersymmetric theories is motivated clearly by the need to reconcile particle physics models with cosmology. The presence of cosmological constant or dark vacuum energy immediately brings up the issue of compatibility with the hierarchy between supersymmetry breaking scale and the size of the vacuum energy density of the Universe. In supersymmetric quintessence models such issues may find a natural explanation, given that scalar cosmological fields in cosmology, such as quintessence fields

\footnotetext{
${ }^{5}$ The cosmological constant problem in mSUGRA models is not solved. Even if one fine tunes the tree level contributions to be small, however loop corrections result in Planck size contributions to vacuum energy.
} 
and inflatons, may arise naturally in a supersymmetric particle spectrum and it is not unreasonable to expect that the current height of the relevant potential may become compatible with the phenomenologically correct size of supersymmetry breaking (of at most a few $\mathrm{TeV}$ ).

Scalar field models of quintessence typically require that the expectation value of the scalar field today is of order of the Planck Mass, in order to explain the observed acceleration of the Universe. This immediately implies that one should include supergravity corrections to such models (in the case of supersymmetric cosmological models). Brax and Martin [117] were the first to discuss supergravity corrections in the quintessence potential in a supergravity model. They considered a (toy) supergravity model with a superpotential

$$
W(\mathcal{Q})=\Lambda^{\alpha+3} \mathcal{Q}^{-\alpha}
$$

where $\Lambda$ is the (mass) scale of the particle theory ${ }^{6}$, and a flat Kähler potential $K=\mathcal{Q} \mathcal{Q}^{*}$, where $\mathcal{Q}$ from now on denotes a scalar superfield.

The resulting scalar potential for such a model [117] has an F-term of the general form [30]:

$$
V(\mathcal{Q})=F^{2}-e^{\kappa^{2} K} 3 \kappa^{2}|W|^{2}
$$

which in the case of a flat Kahler potential, gives [117]

$$
V(\mathcal{Q})=e^{\frac{\kappa^{2}}{2} \mathcal{Q}^{2}} \frac{\Lambda^{4+\beta}}{\mathcal{Q}^{\beta}}\left(\frac{(\beta-2)^{2}}{4}-(\beta+1) \frac{\kappa^{2}}{2} \mathcal{Q}^{2}+\frac{1}{4} \kappa^{4} \mathcal{Q}^{4}\right)
$$

with $\beta=2(\alpha+1)$, and $\kappa=8 \pi M_{P}^{-2}$ the gravitational constant. Notice that, for $\alpha>0$ which is the case of supersymmetric models, the potential has non-polynomial terms in the quintessence field, which when combined with the exponential term turn out, as we shall discuss below, to be crucial for the correct phenomenology of the model in the present epoch.

However, as it stands, the scalar potential (23) is ill defined for $\langle\mathcal{Q}\rangle=M_{P}$ due to negative contributions. One way out [117] would be the imposition of the constraint that the superpotential vanishes $\langle W\rangle=0$, which eliminates the dangerous negative terms in the scalar potential.

The model has several attractive features then. The presence of non-polynomial terms in the scalar potential (23) implies compatibility of the model with a current era acceleration of the Universe. Moreover, due to the presence of the exponential term, the value of the equation of state, $\omega_{Q}$, computed from (20) is pushed towards the value -1 in contrast to the usual non supersymmetric case for which it is difficult to go beyond $\omega_{Q} \approx-0.7$. For $\Omega_{\mathrm{m}} \approx 0.3$, as suggested by the current data, the model of [117] predicts $\omega_{Q} \approx-0.82$, which

\footnotetext{
${ }^{6}$ For instance, in supersymmetric $\mathrm{SU}(\mathrm{N})$ theories $\Lambda$ is the scale at which the gauge coupling becomes non perturbative 118 .
} 

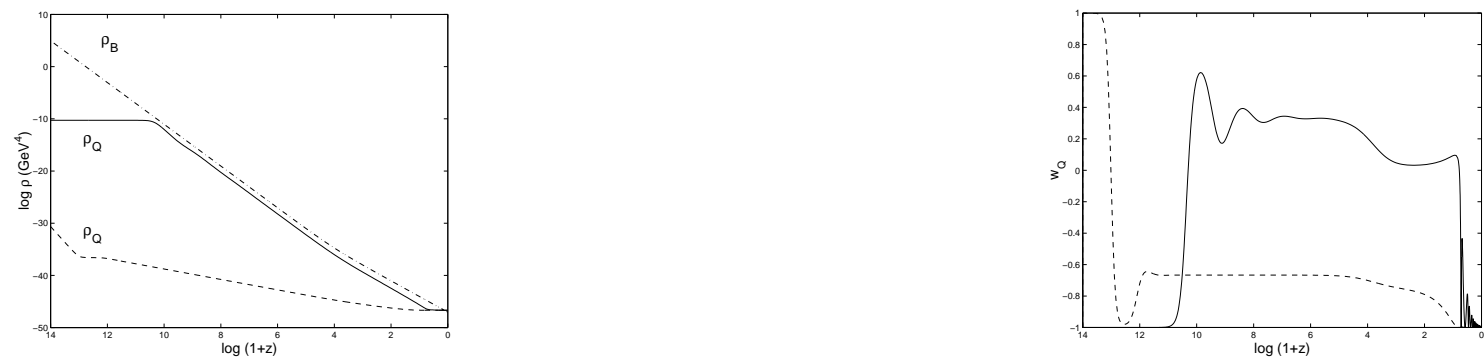

Figure 34: Left: The evolution of the energy density of the quintessence field in the model of ref. [119] versus that of the background ( $z$ is the redshift). Right: The evolution of the equation of state of the quintessence field in that model. In the figures, the dash-dotted line represents the background energy density, the continuous lines correspond to the initial condition for the quintessence field $\mathcal{Q}_{i n} / \mathcal{Q}_{m} \gg 1$, while the dashed lines correspond to the initial condition for the quintessence field $\mathcal{Q}_{i n} / \mathcal{Q}_{m} \ll 1$ (where $\mathcal{Q}_{m}$ denotes the value of the quintessence field at the minimum of the scalar potential).

is in agreement with the recent WMAP data implying $-1<w_{\mathcal{Q}}<-0.78$ (see figures 9), as discussed in section 2 .

The condition $\langle W\rangle=0$ is possible to realise in the presence of other matter fields but in general is a tight restriction. Moreover, such a constraint is not compatible with models of supersymmetry breaking. Things therefore might have been easier if this restriction is relaxed. In ref. [119] a different Kähler potential has been used than in [117]:

$$
K=\left[\ln \left(\kappa \mathcal{Q}+\kappa \mathcal{Q}^{*}\right)\right]^{2} / \kappa^{2} .
$$

The resulting scalar potential has the form:

$$
V=M^{4}\left(2 x^{2}+(4 \alpha-7) x+2(\alpha-1)^{2}\right) \frac{1}{x} e^{\left[(1-x)^{2}-2 \alpha(1-x)\right]}
$$

where $x \equiv\left(-\frac{3}{2} \kappa \mathcal{Q}\right)^{2 / 3}$, and $M^{4}=\Lambda^{6+2 \alpha} \kappa^{2+2 \alpha} 2^{2 \alpha}$. In this case the minimum of the resulting scalar potential is always positive for $\alpha>1.25$ without the need for the imposition of any constraint on $\langle W\rangle$.

The cosmology of that model is given in figure 34. It is important to notice that the presence of field $\mathcal{Q}$ denominators in the potential (25) accompanying the exponential terms are crucial for yielding a negative equation of state $w<0$ in the current era, thereby implying acceleration of the Universe today, as seems to be predicted by observations [1, 3]. Indeed, a solution for the potential involving only exponential and polynomial factors of the quintessence fields cannot yield a negative $w$ today ${ }^{7}$. After reaching its minimum the

\footnotetext{
${ }^{7}$ We note at this stage that the case with only exponential factors of the quintessence field in the
} 
scalar field will mimic the cosmological constant with $w_{\mathcal{Q}}=-1$. At present, the WMAP data point towards $w_{c a l} \rightarrow-1$, which is certainly a feature that characterizes supergravity models (c.f. figure 34), but the big question remains as to whether one can distinguish (supersymmetric) quintessence with $w \rightarrow-1$ from a cosmological constant model. This question is associated clearly with the topic of the present review. If one constructs an appropriate supergravity model of quintessence which can incorporate consistently the dark energy component of the Universe with a broken supersymmetry at TeV scale then one may use such models to constrain particle physics supersymmetry searches as done above for the MSSM. From such studies one may eventually make predictions that could distinguish a quintessence model from a naive cosmological constant one. This remains the biggest challenge for the years to come. The reason is the following.

At this stage it is worth mentioning that the authors of 119 have also considered another type of Kahler potentials,

$$
K=-\frac{1}{\kappa^{2}}\left[\ln \left(\kappa \mathcal{Q}+\kappa \mathcal{Q}^{*}\right)\right] .
$$

Such logarithmic potentials are characteristic of no-scale supergravity models [121], which are known to be derived at low energies from string models [122], as well as from Mtheory 123.

However, the resulting scalar potential, in terms of canonically normalized fields of the form $\tilde{Q}=(\ln \kappa Q) / \sqrt{2} \kappa$, reads:

$$
V(\tilde{\mathcal{Q}})=\left(\Lambda^{5+\beta} \kappa^{1+\beta} \frac{\left(\beta^{2}-3\right)}{2}\right) e^{-\sqrt{2} \beta \kappa \tilde{\mathcal{Q}}}
$$

with $\beta=2 \alpha+1$. Positivity of the potential is guaranteed for $\beta>\sqrt{3}$.

The problem with this model, however, is that, as it stands, the potential (27) has the form of the above-mentioned scaling solution of quintessence [120, according to which one cannot have a current era acceleration of the Universe, since one cannot obtain a negative equation of state. We note, however, that such a conclusion is valid only if there is one quintessence field. As we shall discuss later [17, in more complicated string models, with several field configurations, one has the possibility of obtaining a current era acceleration and a negative value of the equation of state parameter $w$, by appropriately arranging couplings and field magnitudes. Thus, it may well be that no-scale SUGRA models can

potential is discussed in [120, and in this case the attractor is a 'scaling one'. However these results apply in field theory where there is only a single quintessence field, and may not characterise the case of multifield configurations in more complex situations, such as string theory. Indeed, as we shall discuss below, a negative quintessence equation of state today $w<0$ can be obtained for exponential dilaton potentials, at least in a non-critical string cosmology framework [15, 17, 18, 19, where the vacuum energy scale is set by the central charge deficit of the non-critical $\sigma$-model [16], and expresses physically a departure from equilibrium, due to cosmically catastrophic events in our world. 
still provide realistic, phenomenologically acceptable, cosmologies to date. This remains to be seen.

A serious problem for all the supersymmetric quintessence models so far is Supersymmetry Breaking $(S S B)$ at a phenomenologically acceptable scale $M_{S}$, such that $M_{S}^{2} \sim<$ $F>\gtrsim\left(10^{10} \mathrm{GeV}\right)^{2}$ for gravity-mediated cases of SSB, or $M_{S}^{2} \sim<F>\gtrsim\left(10^{4} \mathrm{GeV}\right)^{2}$ for gauge-mediated cases (with $F$ denoting an F-term contribution, see (22)). In the model of [119] this requires a value for $W \sim<F>\kappa^{-1} \sim m_{3 / 2} \kappa^{-2}$, where $m_{3 / 2}$ is the gravitino mass, in order to cancel the F-term contribution and give negligible vacuum energy, in agreement with the known limits (or recent observations) on the cosmological constant (we remind the reader that, in this formalism, the cosmological constant is given by $\left\langle F^{2}\right\rangle$, c.f. (22) ). Although the relaxation of the $\langle W\rangle=0$ constraint allows for such solutions, however it is clear that, in such models, the dynamical cosmological constant provided by the quintessence field cannot be the dominant source of SUSY breaking. The authors of [119] modified their superpotential by adding the term $m_{3 / 2} \kappa^{-2}$ on the right hand side of (21) but then the scalar potential acquires changes $\delta V$ containing this constant term,

$$
\delta V \sim \Lambda^{3+\alpha} m_{3 / 2} \kappa^{-\alpha}+m_{3 / 2}^{2} \kappa^{-2}
$$

This leads to a serious disruption of the quintessence potential, due to the constant second term.
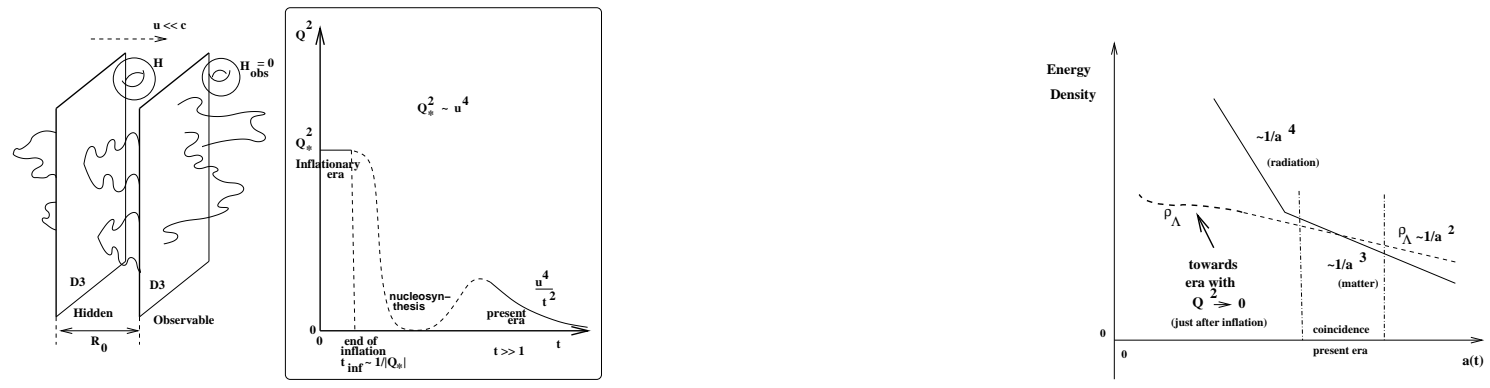

Figure 35: Various non-critical string scenaria of quintessence Left: A non-critical (Liouville) string scenario for quintessence involving colliding five-brane worlds, compactified on magnetized tori [18, 19]. In this model the dilaton field today is constant (with vev of order of the Planck scale), while it was not constant in inflation era. The dashed lines in the evolution of the world-sheet central charge deficit $Q^{2}$ are conjectural, in order to match the criterion of consistency with nucleosynthesis. The continuous lines have been calculated using world-sheet (logarithmic) conformal field theory methods. Right: The evolution of the energy density of the quintessence field in the noncritical string model of Diamandis et al. [17] versus that of the background. In that model, there is a time dependent dilaton on the observable brane world, whose time dependence today is logarithmic, and causes the appearance of a relaxing to zero effective vacuum energy $\Lambda(t) \sim M_{P}^{4} e^{2 \Phi}, \Phi \sim-\ln t$. 
This is still a serious unresolved problem on field theoretic quintessence models. It may well be that string theory models tackle such problems due to unconventional supersymmetry breaking mechanisms one may devise in that framework, as a result of the extra dimensions. For instance, it is advocated in 124 that one may actually live in a four-dimensional world with unbroken supersymmetry, and that the mass splittings between the superpartners occur as a result of the obstruction of supersymmetry, through some higher dimensional effects in string/brane theory. By 'obstruction' we mean here a situation in which the ground state of the system is still supersymmetric, characterised by a zero cosmological constant, but the excitations of the system exhibit supersymmetry breaking mass splittings. If this is the case, then one does not have to worry about supersymmetry breaking terms in the effective SUGRA quintessence potential. However, a concrete realisation of these ideas, in the context of realistic models, is still lacking. Another uncnventional idea about SSB is advocated in ref. [125], according to which the relation between the supersymmetry breaking scale $M_{S}$ and the cosmological constant $<F^{2}>$ (c.f. (22) ) may be modified, in such a way that the amount of vacuum energy, claimed to be observed today, is responsible for the (phenomenologically acceptable) supersymmetry breaking. This could be the case if one had ther following relation:

$$
M_{S}=\kappa^{-1}\left(<F>^{2} \kappa^{4}\right)^{\beta},
$$

with $\beta=1 / 8$ instead of the usually assumed $1 / 4$. In this case, one would not have any dangerous F-terms in the SUGRA potential of the order $\kappa^{-2} M_{S}^{2}$, which would disrupt the quintessence potential. At present, however, at least in the context of conventional field or string theory, such a scenario remains only a conjecture.

We now remark that alternative ways out of these problems could be provided by completely unconventional ways of looking at stringy cosmology, namely the approach to quintessence advocated in [17, 18, 19], by means of non-critical string theory [15]. As we shall discuss below, in such a framework one may also obtain in some cases a concrete realisation of (29) with $\beta=1 / 8$.

According to such scenaria, the quintessence field is provided by the Liouville mode, which is the linear in time part of a string theory dilaton. The current acceleration of the Universe is due to an excitation of our brane world, as a consequence of a catastrophic cosmic event. For instance, in the model of [18, 19] such a catastrophic event is provided by the collision of two brane worlds, moving initially with a small relative velocity $u \ll c$ (c.f. figure 35). This results in a departure from criticality in the resulting world-sheet string theory describing excitations on the brane. In more general situations, such as the models of Diamandis et al. 17, such a departure from equilibrium could be due to an initial quantum fluctuation of a single brane world.

The vacuum energy is relaxing to zero as $M_{P}^{4} / t^{2}$ for long times after the collision, or the initial brane fluctuation, and measures essentially the deviation of the excited string 
system from equilibrium. This scaling is obtained by (logarithmic) conformal field theory methods on the world sheet of the string describing excitations of the (observable) brane world. It is worth stressing that in the model of [17, the effective quintessence (dilaton) potential is of an exponential form $V(\Phi) \sim M_{P}^{4} e^{2 \Phi}$, with the dilaton scaling with (large) time as $\Phi \sim-\ln t$. Naively, one is tempted to think that such potentials could not cause a current-era acceleration of the Universe [120, since this potential characterises the socalled 'scaling solution' in cosmology [120]. However, this conclusion is only true if there are no other fields in the problem. As demonstrated in [17, the string models considered there, have a plethora of other fields (moduli and flux fields), whose magnitudes can be arranged in such a way so as to yield a quintessence equation of state with $w \lesssim-0.75$, and thus a current-era acceleration of the Universe, as claimed by the observations [1, 3, 5. At present, however, one could not identify precise dynamical reasons for such arrangements in the magnitude and the couplings of the various field configurations, and hence, at present, such models may be considered as fine-tuned.

In the above-described models of non-critical string quintessence the basic source of supersymmetry breaking is independent of the value of the vacuum energy today. For instance, in Diamandis et al. [17, one uses type-0 strings which are not supersymmetric due to appropriately projecting out superpartners in the string spectrum [126. On the other hand, in the models of [18, 19], involving five-brane worlds, the supersymmetry breaking is due to the compactification of the two extra dimensions on internal manifolds with magnetic fields. The supersymmetry breaking in this case is obtained as a consequence [127] of the mass splittings in the spectrum of string excitations due to the different coupling of fermionic and bosonic excitations on the brane world to the internal magnetic fields (the associated Nielsen-Olesen instabilities of such scenaria are welcome when the model is considered in a cosmological setting). In fact in some cases within the models of [18 it is even possible to realise concretely the scenario of [125], given that one can derive the relation (29) with $\beta=1 / 8$, although we stress such a relation is not the only phenomenologically consistent solution in these models. As remarked in [19, however, the biggest challenge for such non-critical string theory models of quintessence is the nucleosynthesis era, which seems to require an almost critical string setting (c.f. the left picture in figure 351).

Finally, we wish simply to mention that, in the modern context of brane theories, there are new interesting approaches to the cosmological constant problem, without, however, offering any solution, at present. These explore either the holographic principle, by means of a conformal-field-theory (CFT)/de-Sitter-space (positive cosmological constant) correspondence (in the line of the correspondence between CFT and Anti-de-Sitter Space (with negative cosmological constant) of Maldacena [128), or condensation of tachyons in (nonsupersymmetric) string theory/brane models ${ }^{8}$. Although we think of such issues as very

\footnotetext{
${ }^{8}$ We note that tachyonic instabilities may be useful in cosmology, as triggering inflationary expansion
} 
interesting and intellectually challenging, however, we shall not touch upon them here, due to lack of space. We refer the reader to the relevant literature for more details [129].

This completes our discussion on dark energy and its currently unsolved problems. From our brief but hopefully comprehensive discussion in this section, the reader must have certainly realized that the problem is far from being solved, however it presents many interesting possibilities for future work, both theoretical and experimental. Theoretically, the biggest challenge is to find a correct model that accounts for the currently observed acceleration of the Universe, compatible with other constraints from particle physics, such as supersymmetry breaking. Such a model can then be used to constrain supersymmetric particle physics models, in a similar spirit to mSUGRA discussed here. Experimentally, it is desirable to have more direct evidence on the current acceleration of the Universe by having more dedicated supernovae (or even other type) experiments. This will provide the only direct evidence for such a phenomenon, which when combined with indirect evidence from CMB anisotropies, will give a definite result on the important issue of dark energy.

\section{CONCLUSIONS AND OUTLOOK}

In this article we have given a comprehensive review of the latest constraints on CMSSM, especially after the first data released by the WMAP satellite experiment. Specifically, we have combined the new WMAP cosmological data 3] on Dark Matter with recent high energy physics experimental information including measurements of the anomalous magnetic moment of the muon, from E821 Brookhaven experiment, the $b \rightarrow s \gamma$ branching ratio and the light Higgs boson mass bound from LEP and we studied the imposed constraints on the parameter space of the CMSSM. We have assessed the potential of discovering SUSY, if it is based on CMSSM, at future colliders and DM direct search experiments. The available parameter space seems to be constrained severely by the combination of the above-mentioned data. The use of the new WMAP data in conjunction with the $2 \sigma$ $\left(g_{\mu}-2\right)$ bound can guarantee that in LHC but also in a $e^{+} e^{-}$collider with center of mass energy $\sqrt{s} \approx 1.1 \mathrm{TeV}$ CMSSM can be discovered provided that the high (inversion) region of the HB is not realized.

However, WMAP data seem to favour HB/focus point region (this was based on a $\chi^{2}$ analysis of mSUGRA model). Updated reach studies have indicated that, for an integrated luminosity $100 \mathrm{fb}^{-1}$, LHC can probe $m_{1 / 2} \sim 1400 \mathrm{GeV}$, for small $m_{0}$ (gluino mass $m_{\tilde{g}} \sim 3$ $\mathrm{TeV}$ ). For large $m_{0}$ the $\mathrm{HB} /$ focus point region $m_{1 / 2} \sim 700 \mathrm{GeV}$ can be probed (gluino mass $m_{\tilde{g}} \sim 1800 \mathrm{GeV}$ ) via conventional SUSY channels.

However HB/focus point region appears to extent indefinitely to large $m_{1 / 2}, m_{0}$ values, far beyond LHC reach. Such regions can be probed by direct dark mater searches.

of the Universe in the past, but being completely harmless today 17 129]. 
The effect of these constraints is also significant for the direct DM searches. For the $\mu>0$ case we found that the minimum value of the spin-independent $\chi_{1}^{0}$-nucleon cross section attained is of the order of $10^{-9} \mathrm{pb}[69$.

We have also discussed such constraints, combined with those coming from proton life time current lower limts, in the context of grand unified supersymmetric models, specifically the flipped SU(5) model, which seems to survive such tight constraints.

For future directions we point out that one should take seriously into account the other constraint coming from recent astrophysical data, that of dark energy. We believe it is quintessence, i.e a relaxing to zero (non-equilibrium) field. The WMAP data point towards an equation of state of quintessence $w=p / \rho \rightarrow-1$ which is close to that of a cosmological constant. This feature is shared by Quintessence SUGRA models [117, and should be explored further within Superstring, Brane models of a relaxing to zero vacuum energy, in conjunction with the issue of supersymmetry breaking.

The important theoretical task for the years to come will be to determine the (physically) correct SUGRA/string/brane model to constrain SUSY searches exploiting the non-zero Dark Energy component of Universe. Experimentally, it is certain that the postWMAP era, which now begins, will imply that Particle Physics and Astrophysics will proceed together for the exciting years to come and provide useful and complementary physics input to each other.

\section{ACKNOWLEDGMENTS}

We wish thank D.Ahluwalia-Khalilova for the invitation to write this review for Int. J. Mod. Phys. D, which was based on numerous presentations by the authors, including a plenary talk (NEM) at the 4th ATLAS Physics Workshop, Athens University (Greece), May 21-25 2003. NEM wishes to thank Fabiola Gianotti for the invitation in that meeting, and acknowledges useful discussions with her and Jim Pinfold. It is also a great pleasure to thank J. Walker for providing us with material on proton decay prior to publication, which we used in this review. The work of A.B.L. is partially supported by the European Union (contracts HPRN-CT-2000-00148 and HPRN-CT-2000-00149), and by the University of Athens Research Committee. That of N.E.M. is partially supported by a visiting professorship at the Theoretical Physics Department of the University of Valencia (Spain), and by the European Union (contract HPRN-CT-2000-00152). The work of D.V.N. is supported by D.O.E. grant DE-FG03-95-ER-40917.

\section{References}

[1] B. P. Schmidt et al., Astrophys. J. 507 (1998) 46 arXiv:astro-ph/9805200; S. Perlmutter et al. [Supernova Cosmology Project Collaboration], Astrophys. J. 517 
(1999) 565 arXiv:astro-ph/9812133 ; J. P. Blakeslee et al., Astrophys. J. 589 (2003) 693 arXiv:astro-ph/0302402; A. G. Riess et al., Astrophys. J. 560 (2001) 49 arXiv:astro-ph/0104455.

[2] S. Perlmutter and B. P. Schmidt, arXiv:astro-ph/0303428, J. L. Tonry et al., arXiv:astro-ph/0305008

[3] C. L. Bennett et al., arXiv:astro-ph/0302207

[4] G. F. Smoot et al., Astrophys. J. 396, L1 (1992); C. L. Bennett et al., Astrophys. J. 436, 423 (1994) arXiv:astro-ph/9401012;

[5] D. N. Spergel et al., arXiv:astro-ph/0302209.

[6] J. R. Ellis, J. S. Hagelin, D. V. Nanopoulos, K. A. Olive and M. Srednicki, Nucl. Phys. B238 (1984) 453; H. Goldberg, Phys. Rev. Lett. 50 (1983) 1419.

[7] E. Kolb abd M. Turner, The Early Universe (Frontiers in Physics, Addison-Wesley, 1989).

[8] H. V. Peiris et al., arXiv:astro-ph/0302225, V. Barger, H. S. Lee and D. Marfatia, Phys. Lett. B 565, 33 (2003) arXiv:hep-ph/0302150.

[9] A. H. Guth, Phys. Rev. D 23 (1981) 347; A. D. Linde, Phys. Lett. B 108 (1982) 389; A. Albrecht and P. J. Steinhardt, Phys. Rev. Lett. 48 (1982) 1220. for reviews see: K. A. Olive, Phys. Rept. 190 (1990) 307; D. H. Lyth and A. Riotto, Phys. Rept. 314 (1999) 1 arXiv:hep-ph/9807278.

[10] D. V. Nanopoulos, K. A. Olive and M. Srednicki, Phys. Lett. B 127 (1983) 30.

[11] A. Kosowsky and M. S. Turner, Phys. Rev. D 52, 1739 (1995) arXiv:astro-ph/9504071.

[12] W. H. Kinney, E. W. Kolb, A. Melchiorri and A. Riotto, arXiv:hep-ph/0305130

[13] H. Murayama, H. Suzuki, T. Yanagida and J. Yokoyama, Phys. Rev. Lett. 70, 1912 (1993); H. Murayama, H. Suzuki, T. Yanagida and J. Yokoyama, Phys. Rev. D 50, 2356 (1994) arXiv:hep-ph/9311326; J. R. Ellis, M. Raidal and T. Yanagida, arXiv:hep-ph/0303242

[14] R. Brustein, M. Gasperini, M. Giovannini, V. F. Mukhanov and G. Veneziano, Phys. Rev. D 51, 6744 (1995) arXiv:hep-th/9501066. For a review see: R. Brustein, arXiv:hep-th/9506045, and references therein. 
[15] J. R. Ellis, N. E. Mavromatos and D. V. Nanopoulos, Mod. Phys. Lett. A 10 (1995) 1685 arXiv:hep-th/9503162.

[16] F. David, Mod. Phys. Lett. A 3 (1988) 1651; . Distler and H. Kawai, Nucl. Phys. B 321 (1989) 509; see also: N. E. Mavromatos and J. L. Miramontes, Mod. Phys. Lett. A 4 (1989) 1847; E. D'Hoker and P. S. Kurzepa, Mod. Phys. Lett. A 5 (1990) 1411; I. R. Klebanov, I. I. Kogan and A. M. Polyakov, Phys. Rev. Lett. 71 (1993) 3243 arXiv:hep-th/9309106.

[17] J. R. Ellis, N. E. Mavromatos and D. V. Nanopoulos, Gen. Rel. Grav. 32 (2000) 943 arXiv:gr-qc/9810086; G. A. Diamandis, B. C. Georgalas, N. E. Mavromatos, E. Papantonopoulos and I. Pappa, Int. J. Mod. Phys. A 17 (2002) 2241 arXiv:hep-th/0107124 ; G. A. Diamandis, B. C. Georgalas, N. E. Mavromatos and E. Papantonopoulos, Int. J. Mod. Phys. A 17 (2002) 4567 arXiv:hep-th/0203241;

[18] E. Gravanis and N. E. Mavromatos, Phys. Lett. B 547 (2002) 117 arXiv:hep-th/0205298;

[19] N. E. Mavromatos, arXiv:hep-th/0210079

[20] J. R. Ellis, N. E. Mavromatos and D. V. Nanopoulos, Phys. Lett. B 293 (1992) 37 arXiv:hep-th/9207103; for reviews see: J. R. Ellis, N. E. Mavromatos and D. V. Nanopoulos, Erice Subnucl. Phys. Series, Vol. 311 (World Sci. 1994) arXiv:hep-th/9311148; J. Chaos, Solitons and Fractals, Vol. 10 (1999) 345 (eds. C. Castro amd M.S. El Naschie, Elsevier Science, Pergamon 1999) arXiv:hep-th/9805120.

[21] I. I. Kogan, Prepared for Particles 8 Fields 91: Meeting of the Division of Particles $\&$ Fields of the APS, Vancouver, Canada, 18-22 Aug 1991; Phys. Lett. B 265 (1991) 269.

[22] I. Antoniadis, C. Bachas, J. R. Ellis and D. V. Nanopoulos, Phys. Lett. B 211 (1988) 393; Nucl. Phys. B 328 (1989) 117; "Comments On Cosmological String Solutions," Phys. Lett. B 257 (1991) 278.

[23] N. Yoshida, A. Sokasian, L. Hernquist and V. Springel, Astrophys. J. 591, L1 (2003) arXiv:astro-ph/0303622.

[24] J. R. Ellis, J. L. Lopez and D. V. Nanopoulos, Phys. Lett. B 247 (1990) 257; J. R. Ellis, G. B. Gelmini, J. L. Lopez, D. V. Nanopoulos and S. Sarkar, Nucl. Phys. B 373 (1992) 399; P. Gondolo, G. Gelmini and S. Sarkar, Nucl. Phys. B 392 (1993) 111 arXiv:hep-ph/9209236. V. Berezinsky, M. Kachelriess and A. Vilenkin, Phys. Rev. Lett. 79 (1997) 4302 arXiv:astro-ph/9708217. V. A. Kuzmin and 
V. A. Rubakov, Phys. Atom. Nucl. 61 (1998) 1028 [Yad. Fiz. 61 (1998) 1122] arXiv:astro-ph/9709187; K. Benakli, J. R. Ellis and D. V. Nanopoulos, Phys. Rev. D 59 (1999) 047301 arXiv:hep-ph/9803333; M. Birkel and S. Sarkar, Astropart. Phys. 9 (1998) 297 arXiv:hep-ph/9804285; G. Gelmini and A. Kusenko, Phys. Rev. Lett. 84 (2000) 1378 arXiv:hep-ph/9908276; J. L. Crooks, J. O. Dunn and P. H. Frampton, Astrophys. J. 546 (2001) L1 arXiv:astro-ph/0002089; S. Sarkar, arXiv:hep-ph/0005256.

[25] D. J. Chung, Phys. Rev. D 67 (2003) 083514 arXiv:hep-ph/9809489.

[26] J. R. Ellis, S. Kelley and D. V. Nanopoulos, Phys. Lett. B249 (1990) 441; Phys. Lett. B260 (1991) 131; U. Amaldi, W. de Boer and H. Furstenau, Phys. Lett. B260 (1991) 447; C. Giunti, C. W. Kim and U. W. Lee, Mod. Phys. Lett. A6 (1991) 1745.

[27] LEP Electroweak Working Group, http://lepewwg.web.cern.ch/LEPEWWG/Welcome.html

[28] J. R. Ellis, T. Falk, K. A. Olive and M. Srednicki, Astropart. Phys. 13 (2000) 181 [Erratum-ibid. 15, 413 (2001)] arXiv:hep-ph/9905481.

[29] A. H. Chamseddine, R. Arnowitt and P. Nath, Phys. Rev. Lett. 49 (1982) 970; R. Barbieri, S. Ferrara and C. A. Savoy, Phys. Lett. B 119 (1982) 343; L. J. Hall, J. Lykken and S. Weinberg, Phys. Rev. D 27 (1983) 2359; P. Nath, R. Arnowitt and A. H. Chamseddine, Nucl. Phys. B 227 (1983) 121.

[30] For reviews see: H. P. Nilles, Phys. Rept. 110 (1984) 1; A. B. Lahanas and D. V. Nanopoulos, Phys. Rept. 145 (1987) 1.

[31] R. Arnowitt and P. Nath, Phys. Rev. D 46 (1992) 398.

[32] K. L. Chan, U. Chattopadhyay and P. Nath, Phys. Rev. D 58 (1998) 096004 arXiv:hep-ph/9710473.

[33] U. Chattopadhyay, A. Corsetti and P. Nath, arXiv:hep-ph/0303201.

[34] L. E. Ibanez, C. Lopez and C. Munoz, Nucl. Phys. B 256 (1985) 218.

[35] J. L. Feng, K. T. Matchev and F. Wilczek, Phys. Lett. B 482 (2000) 388 arXiv:hep-ph/0004043; Phys. Rev. D 63 (2001) 045024 arXiv:hep-ph/0008115.

[36] J. L. Feng, K. T. Matchev and T. Moroi, Phys. Rev. Lett. 84 (2000) 2322 arXiv:hep-ph/9908309; Phys. Rev. D 61 (2000) 075005 arXiv:hep-ph/9909334. 
[37] J. R. Ellis, T. Falk and K. A. Olive, Phys. Lett. B 444 (1998) 367; J. R. Ellis, T. Falk, K. A. Olive and M. Srednicki, Astropart. Phys. 13 (2000) 181 [Erratum-ibid. 15, 413 (2001)] arXiv:hep-ph/9905481.

[38] M. E. Gomez,G. Lazarides and C. Pallis, Phys. Rev. D 61 (2000) 123512.

[39] L. Roszkowski, R. Ruiz de Austri and T. Nihei, JHEP 0207 (2002) 024.

[40] J. Edsjo and P. Gondolo, Phys. Rev. D 56 (1997) 1879 arXiv:hep-ph/9704361.

[41] H. Baer and C. Balazs, arXiv:hep-ph/0303114

[42] M. Srednicki, R. Watkins and K.A. Olive, Nucl. Phys. B 310 (1988) 693.

[43] J. Ellis, D.V. Nanopoulos, L. Roszkowski and D. N. Schramm, Phys. Lett. B 245 (1990) 251; J. Ellis, L. Roszkowski and Z. Lalak, Phys. Lett. B 245 (1990) 545; L. Roszkowski Phys. Lett. B 252 (1990) 471; Phys. Lett. B 262(1991) 59; J. Ellis and L. Roszkowski, Phys. Lett. B 283 (1992) 252; L. Roszkowski and R. Roberts, Phys. Lett. B 309 (1993) 329; G.L. Kane, C. Kolda, L. Roszkowski and J.D. Wells, Phys. Rev. D 49 (1994) 6173.

[44] K.A. Olive and M. Srednicki, Phys. Lett. B 230(1989) 78; Nucl. Phys. B 355 (1991) 208; K. Griest, M. Kamionkowski and M. S. Turner, Phys. Rev. D 41(1990) 3565; J. McDonald, K. A. Olive and M. Srednicki, Phys. Lett. B 283 (1992) 80; S. Mizuta, D. Ng and M. Yamaguchi, Phys. Lett. B 300 (1993) 96.

[45] J. Ellis and F. Zwirner, Nucl. Phys. B 338 (1990) 317; M. M. Nojiri, Phys. Lett. B 261 (1991) 76; M. Kawasaki and S. Mizuta, Phys. Rev. D 46 (1992) 1634.

[46] J. L. Lopez, D. V. Nanopoulos and K. Yuan, Phys. Lett. B 267 (1991) 219; J. L. Lopez, D. V. Nanopoulos, H. Pois and K. Yuan, Phys. Lett. B 273(1991) 423; J. L. Lopez, D. V. Nanopoulos and K. Yuan, Nucl. Phys. B 370 (1992) 445; Phys. Rev. D 48(1993) 2766; S. Kelley, J. L. Lopez, D. V. Nanopoulos, H. Pois and K. Yuan, Phys. Rev. D 47 (1993) 2461.

[47] R. Arnowitt and P. Nath, Phys. Rev. Lett. 70 (1993) 3696; Phys. Rev. D 54 (1996) 2374; M. Drees and A. Yamada, Phys. Rev. D 53 (1996) 1586; J. Ellis, T. Falk, K. A. Olive and M. Schmitt, Phys. Lett. B 388 (1996) 97.

[48] J. Ellis, T. Falk, G. Ganis and K.A. Olive, Phys. Rev. D 58 (1998) 095002.

[49] G. Jungman, M. Kamionkowski and K. Griest, Phys. Rept. 267 (1996) 195; 
[50] K. Griest, Phys. Rev. D 38 (1988) 2357 [Erratum: 39 (1989) 3802]. J. Ellis and R. Flores, Nucl. Phys. B 307 (1988) 833. R. Barbieri, M. Frigeni and G. Guidice, Nucl. Phys. B 313 (1989) 725. K. Griest and D. Seckel, Phys. Rev. D 43 (1991) 3191. P. Gondolo and G. Gelmini, Nucl. Phys. B 360 (1991) 145. A. Bottino et. al., Phys. Lett. B 295 (1992) 330; M. Drees and M. M. Nojiri, Phys. Rev. D 48 (1993) 3483; V. A. Bednyakov, H. V. Klapdor-Kleingrothaus and S. Kovalenko, Phys. Rev. D 50 (1994) 7128; P. Nath and R. Arnowitt, Phys. Rev. Lett. D 74 (1995) 4592; E. Diehl, G. L. Kane, C. Kolda and J. D. Wells, Phys. Rev. D 52 (1995) 4223; R. Arnowitt and P. Nath, Phys. Rev. D 54 (1996) 2374; L. Bergstrom and P. Gondolo, Astropart. Phys. 6 (1996) 263; G. Jungman, M. Kamionkowski and K. Griest, Phys. Rept. 267 (1996) 195; H. Baer and M. Brhlik, Phys. Rev. D 53 (1996) 597; M. Drees, M. M. Nojiri, D. Roy and Y. Yamada, Phys. Rev. D 56 (1997) 276; V. Barger and C. Kao, Phys. Rev. D 57 (1998) 3131. H. Baer and M. Brhlik, Phys. Rev. D 57 (1998) 567; J. D. Vergados, Phys. Rev. D 83 (1998) 3597; J. Wells, Phys. Lett. B4 43 (1998) 196. M. Brhlik, D. J. Chung and G. L. Kane, Int. J. Mod. Phys. D 10 (2001) 367; V. A. Bednyakov and H. V. Klapdor-Kleingrothaus, Phys. Rev. D 63 (2001) 095005; M. E. Gomez and J. D. Vergados, Phys. Lett. B 512 (2001) 252; V. D. Barger and C. Kao, Phys. Lett. B 518 (2001) 117; L. Roszkowski, R. Ruiz de Austri and T. Nihei, JHEP 0108 (2001) 024; H. Baer, C. Balazs, A. Belyaev, J. K. Mizukoshi, X. Tata and Y. Wang, JHEP 0207 (2002) 050; U. Chattopadhyay and P. Nath, Phys. Rev. D 66 (2002) 093001;

[51] P. Nath and R. Arnowitt, Phys. Rev. D 56 (1997) 2820; J. Ellis, A. Ferstl, K. A. Olive and Y. Santoso, arXiv:hep-ph/0302032 A. Corsetti and P. Nath, Phys. Rev. D 64 (2001) 125010; hep-ph/0005234 hep-ph/0011313 A. Birkedal-Hansen and B. D. Nelson, arXiv:hep-ph/0211071.

[52] U. Chattopadhyay, T. Ibrahim and P. Nath, Phys. Rev. D 60 (1999) 063505 ; S. Khalil and Q. Shafi, Nucl. Phys. B 564 (1999) 19; K. Freese and P. Gondolo, hep-ph/9908390, S. Y. Choi, hep-ph/9908397. T. Falk, A. Ferstl and K. A. Olive, Astropart. Phys. 13 (2000) 301; S. Khalil, Phys. Lett. B 484 (2000) 98.

[53] U. Chattopadhyay and P. Nath, Phys. Rev. D $61 \quad$ (2002) 035003 arXiv:hep-ph/0201001; U. Chattopadhyay, A. Corsetti and P. Nath, Phys. Rev. D 65 (2002) 075009 arXiv:hep-ph/0110341.

[54] P. Belli, R. Bernbei, A. Bottino, F. Donato, N. Fornengo, D. Prosperi and S. Scopel, Phys. Rev. D 61 (2000) 023512; M. Brhlik and L. Roszkowski, Phys. Lett. B 464 (1999) 303; A. Corsetti and P. Nath, Int. J. Mod. Phys. A 15 (2000) 905.

[55] S. Mizuta and M. Yamaguchi, Phys. Lett. B 298 (1993) 120. 
[56] R. Arnowitt, B. Dutta and Y. Santoso, Nucl. Phys. B 606 (2001) 59 [arXiv: hep-ph/0102181; J. R. Ellis, K. A. Olive and Y. Santoso, arXiv: hep-ph/0112113.

[57] V. A. Bednyakov, H. V. Klapdor-Kleingrothaus and V. Gronewold, arXiv: hep-ph/0208178

[58] A. B. Lahanas, D. V. Nanopoulos and V. C. Spanos, Phys. Rev. D62 (2000) 023515.

[59] A. B. Lahanas, D. V. Nanopoulos and V. C. Spanos, Mod. Phys. Lett. A16 (2001) 1229.

[60] A. B. Lahanas, D. V. Nanopoulos and V. C. Spanos, arXiv:hep-ph/0112134 and hep-ph/0211286,

[61] A. B. Lahanas, D. V. Nanopoulos and V. C. Spanos, Phys. Lett. B 518 (2001) 94 arXiv:hep-ph/0107151.

[62] M. Drees and M. Nojiri, Phys. Rev. D47 (1993) 376; R. Arnowitt and P. Nath, Phys. Lett. B299 (1993) 58.

[63] M. Drees, Y. G. Kim, T. Kobayashi and M. M. Nojiri, Phys. Rev. D63 (2001) 115009.

[64] For a recent review see: J. R. Ellis, arXiv:astro-ph/0305038, and references therein.

[65] M. Battaglia, A. De Roeck, J. R. Ellis, F. Gianotti, K. T. Matchev, K. A. Olive, L. Pape and G. Wilson, Eur. Phys. J. C 22 (2001) 535 arXiv:hep-ph/0106204; also in Proc. of the APS/DPF/DPB Summer Study on the Future of Particle Physics (Snowmass 2001) ed. N. Graf, eConf C010630 (2001) P347 arXiv:hep-ph/0112013.

[66] G. W. Bennet et. al.[BNL-E821 Collaboration], Phys. Rev. Lett. 89 (2002) 101804; C. J. Onderwater et al. [BNL-E821 Collaboration], AIP Conf. Proc. 549 (2002) 917.

[67] S. Narison, arXiv:hep-ph/0303004.

[68] J. R. Ellis, K. A. Olive, Y. Santoso and V. C. Spanos, Phys. Lett. B 565 (2003) 176 arXiv:hep-ph/0303043.

[69] A. B. Lahanas and D. V. Nanopoulos, arXiv:hep-ph/0303130 Phys. Lett. B in press.

[70] A. B. Lahanas and V. C. Spanos, Eur. Phys. J. C23 (2002) 185.

[71] B. de Carlos and J. A. Casas, Phys. Lett. B309 (1993) 320; H. Baer, C. Chen, M. Drees, F. Paige and X. Tata, Phys. Rev. Lett. 76 (1997) 986; J. A. Casas, J. R. Espinosa and H. E. Haber, Nucl. Phys. B526 (1998) 3. 
[72] J. A. Bagger, K. Matchev, D. M. Pierce and R. -J. Zhang, Nucl. Phys. B491 (1997) 3.

[73] R. Hempfling, Phys. Rev. D49 (1994) 6168; L. J. Hall, R. Rattazi and U. Sarid, Phys. Rev. D50 (1994) 7048; R. Rattazi and U. Sarid, Phys. Rev. D53 (1996) 1553.

[74] M. Carena, M. Olechowski, S. Pokorski and C. Wagner, Nucl. Phys. B426 (1994) 269.

[75] E. Accomando, R. Arnowitt, B. Dutta and Y. Santoso, Nucl. Phys. B585 (2000) 124.

[76] H. Eberl, K. Hidaka, S. Kraml, W. Majerotto and Y. Yamada, Phys. Rev. D62 (2000) 055006.

[77] M. Carena, D. Garcia, U. Nierste and C. Wagner, Nucl. Phys. B577 (2000) 88.

[78] G. Degrassi, P. Slavich and F. Zwirner, Nucl. Phys. B611 (2001) 403; A. Brignole, G. Degrassi, P. Slavich and F. Zwirner, Nucl. Phys. B631 (2002) 195; Nucl. Phys. B643 (2002) 79.

[79] M. Ciuchini, G. Degrassi, P. Gambino and G. F. Giudice, Nucl. Phys. B534 (1998) 3; G. Degrassi, P. Gambino and G. F. Giudice, JHEP (2000) 0012:009; M. Carena, D. Garcia, U. Nierste and C. Wagner, Phys. Lett. B499 (2001) 141.

[80] A. Katsikatsou, A. B. Lahanas, D. V. Nanopoulos and V. C. Spanos, Phys. Lett. B501 (2001) 69.

[81] M. Battaglia, A. De Roeck, J. Ellis, F. Gianotti, K. A. Olive and L. Pape, arXiv:hep-ph/0306219

[82] H. Baer, C. Balazs, A. Belyaev, T. Krupovnickas and X. Tata, JHEP 0306 (2003) 054 arXiv:hep-ph/0304303.

[83] H. Baer, T. Krupovnickas and X. Tata, JHEP $\mathbf{0 3 0 7}(2003) \quad 020$ arXiv:hep-ph/0305325.

[84] R. Abusaidi et. al., CDMS Collaboration, Phys. Rev. Lett. 84 (2000) 5699.

[85] R. Bernabei et. al., DAMA Collaboration, Phys. Lett. B480 (2000) 23.

[86] H. V. Klapdor-Kleingrothaus, hep-ph/0104028.

[87] See: C. Hailey, talk at LEAP03, Yokohama (Japan), March 3-7 2003, http://ad3-proj-leap03.web.cern.ch/ad3-proj-leap03/

[88] J. R. Ellis, S. Kelley and D. V. Nanopoulos, Nucl. Phys. B 373 (1992) 55; Phys. Lett. B 287 (1992) 95 arXiv:hep-ph/9206203. 
[89] J. R. Ellis, J. L. Lopez and D. V. Nanopoulos, Phys. Lett. B 371 (1996) 65 arXiv:hep-ph/9510246.

[90] R. Barbieri and L. Hall, Phys. Rev. Lett. 68 (1992) 752; J. Hisano, H. Murayama, and T. Yanagida, Phys. Rev. Lett. 69 (1992) 1014; K. Hagiwara and Y. Yamada, Phys. Rev. Lett. 70 (1993) 709; F. Anselmo, L. Cifarelli, A. Peterman, and A. Zichichi, Nuovo Cim. 105A (1992) 1025; P. Langacker and N. Polonsky, Phys. Rev.47 (1993) 4028.

[91] M. Bastero-Gil and J. Perez-Mercader, Phys. Lett. B 322 (1994) 355; A. Faraggi and B. Grinstein, Nucl. Phys. B 422 (1994) 3; P. Chankowski, Z. Pluciennik and S. Pokorski, Nucl. Phys. B 439 (1995) 23; P. Langacker and N. Polonsky, arXiv:hep-ph/9503214 L. Clavelli and P. Coulter, Phys. Rev. D 51 (1995) 3913; J. Bagger, K. Matchev, and D. Pierce, Phys. Lett. B 348 (1995) 443.

[92] S. Weinberg, Phys. Rev. Lett. 43 (1979) 1566; N. Sakai and T. Yanagida, Nucl. Phys. B 197 (1982) 533.

[93] J. R. Ellis, D. V. Nanopoulos and S. Rudaz, Nucl. Phys. B 202 (1982) 43.

[94] H. Murayama and A. Pierce, Phys. Rev. D 65 (2002) 055009 arXiv:hep-ph/0108104.

[95] M. Shiozawa et al. [Super-Kamiokande Collaboration], Phys. Rev. Lett. 81 (1998) 3319 arXiv:hep-ex/9806014; Y. Hayato et al. [SuperKamiokande Collaboration], Phys. Rev. Lett. 83 (1999) 1529 arXiv:hep-ex/9904020.

[96] K. Hagiwara et al. [Particle Data Group Collaboration], Phys. Rev. D 66 (2002) 010001.

[97] S. M. Barr, Phys. Lett. B 112 (1982) 219; J. P. Derendinger, J. E. Kim and D. V. Nanopoulos, Phys. Lett. B 139 (1984) 170; I. Antoniadis, J. R. Ellis, J. S. Hagelin and D. V. Nanopoulos, Phys. Lett. B 194 (1987) 231.

[98] I. Antoniadis, J. R. Ellis, J. S. Hagelin and D. V. Nanopoulos, Phys. Lett. B 205 (1988) 459; Phys. Lett. B 208 (1988) 209 [Addendum-ibid. B 213 (1988) 562]; Phys. Lett. B 231 (1989) 65.

[99] J. R. Ellis, D. V. Nanopoulos and J. Walker, Phys. Lett. B 550 (2002) 99 arXiv:hep-ph/0205336.

[100] C. K. Jung, arXiv:hep-ex/0005046

[101] J. R. Ellis, K. A. Olive and Y. Santoso, arXiv:hep-ph/0202110. 
[102] S. Heinemeyer, W. Hollik and G. Weiglein, Comput. Phys. Commun. 124 (2000) 76 arXiv:hep-ph/9812320; S. Heinemeyer, W. Hollik and G. Weiglein, Eur. Phys. J. C 9 (1999) 343 arXiv:hep-ph/9812472.

[103] J. R. Ellis, J. S. Hagelin, S. Kelley and D. V. Nanopoulos, Nucl. Phys. B 311 (1988) 1 .

[104] J. R. Ellis, J. L. Lopez, D. V. Nanopoulos and K. A. Olive, Phys. Lett. B 308 (1993) 70 arXiv:hep-ph/9303307.

[105] J. R. Ellis, P. Kanti and D. V. Nanopoulos, Nucl. Phys. B 647 (2002) 235 arXiv:hep-th/0206087.

[106] S. Weinberg, Rev. Mod. Phys. 61 (1989) 1; S. M. Carroll, W. H. Press and E. L. Turner, Ann. Rev. Astron. Astrophys. 30 (1992) 499; arXiv:astro-ph/9610044.

[107] S. Hellerman, N. Kaloper and L. Susskind, JHEP 0106 (2001) 003 arXiv:hep-th/0104180; W. Fischler, A. Kashani-Poor, R. McNees and S. Paban, JHEP 0107 (2001) 003 arXiv:hep-th/0104181; E. Witten, arXiv:hep-ph/0002297; arXiv:hep-th/0106109 P. O. Mazur and E. Mottola, Phys. Rev. D 64 (2001) 104022 arXiv:hep-th/0106151.

[108] J. R. Ellis, N. E. Mavromatos and D. V. Nanopoulos, arXiv:hep-th/0105206;

[109] M. Ozer and M. O. Taha, Phys. Lett. B 171 (1986) 363; M. Reuter and C. Wetterich, Phys. Lett. B 188 (1987) 38; C. Wetterich, Astron. Astrophys. 301 (1995) 321 arXiv:hep-th/9408025; J. L. Lopez and D. V. Nanopoulos, Mod. Phys. Lett. A 11 (1996) 1 arXiv:hep-ph/9501293.

[110] J. L. Lopez and D. V. Nanopoulos, Mod. Phys. Lett. A 9 (1994) 2755 arXiv:hep-ph/9402275.

[111] Only a partial list of references: M. S. Turner and M. White, Phys. Rev. D56 (1997) 4439; J. A. Frieman and I. Waga, Phys. Rev. D57 (1998) 4642; R. R. Caldwell, R. Dave and P. J. Steinhardt, Phys. Rev. Lett. 80 (1998), 1582; L. M. Wang and P. J. Steinhardt, Astrophys. J. 508 (1998) 483 arXiv:astro-ph/9804015; I. Zlatev, L. M. Wang and P. J. Steinhardt, Phys. Rev. Lett. 82 (1999) 896 arXiv:astro-ph/9807002 ; L. M. Wang, R. R. Caldwell, J. P. Ostriker and P. J. Steinhardt, Astrophys. J. 530 (2000) 17 arXiv:astro-ph/9901388; P. J. Steinhardt, Phys. Scripta T85 (2000) 177; Phys. Rev. Lett. 90 (2003) 231302 arXiv:hep-th/0210156; M. Doran and C. Wetterich, arXiv:astro-ph/0205267; P. K. Townsend, JHEP 0111 (2001) 042 arXiv:hep-th/0110072; S. Mizuno and K. i. Maeda, Phys. Rev. D 
64 (2001) 123521 arXiv:hep-ph/0108012 ; A. Albrecht, C. P. Burgess, F. Ravndal and C. Skordis, Phys. Rev. D 65 (2002) 123507 arXiv:astro-ph/0107573; M. C. Bento, O. Bertolami and N. M. Santos, Phys. Rev. D 65 (2002) 067301 arXiv:astro-ph/0106405; P. Brax, J. Martin and A. Riazuelo, Phys. Rev. D 64 (2001) 083505 arXiv:hep-ph/0104240; G. Huey and J. E. Lidsey, Phys. Lett. B 514 (2001) 217 arXiv:astro-ph/0104006; K. i. Maeda, Phys. Rev. D 64 (2001) 123525 arXiv:astro-ph/0012313 ; P. F. Gonzalez-Diaz, Phys. Lett. B 481 (2000) 353 arXiv:hep-th/0002033 ; O. Bertolami and P. J. Martins, Phys. Rev. D 61 (2000) 064007 arXiv:gr-qc/9910056] V. Sahni and L. M. Wang, Phys. Rev. D 62 (2000) 103517 arXiv:astro-ph/9910097; L. Amendola, Phys. Rev. D 62 (2000) 043511 arXiv:astro-ph/9908023 ; M. C. Bento and O. Bertolami, Gen. Rel. Grav. 31 (1999) 1461 arXiv:gr-qc/9905075.

[112] P. J E. Peebles and B. Ratra, Astrophys. J. 325 (1988) L17; B. Ratra and P. J E. Peebles, Phys. Rev. D37 (1988) 3406; A. R. Liddle and R. J. Scherrer, Phys. Rev. D59 (1999) 023509.

[113] M. Gasperini, Phys. Rev. D 64 (2001) 043510 arXiv:gr-qc/0105082; M. Gasperini, F. Piazza and G. Veneziano, Phys. Rev. D 65 (2002) 023508 arXiv:gr-qc/0108016; R. Bean and J. Magueijo, Phys. Lett. B 517 (2001) 177 arXiv:astro-ph/0007199.

[114] R. Wald, Phys. Rev. D21 (1980) 2742.

[115] J. R. Ellis, N. E. Mavromatos and D. V. Nanopoulos, "Testing quantum mechanics in the neutral kaon system," Phys. Lett. B 293 (1992) 142 arXiv:hep-ph/9207268; Int. J. Mod. Phys. A 11 (1996) 1489 arXiv:hep-th/9212057; J. R. Ellis, J. L. Lopez, N. E. Mavromatos and D. V. Nanopoulos, Phys. Rev. D 53 (1996) 3846 arXiv:hep-ph/9505340 ; P. Huet and M. E. Peskin, Nucl. Phys. B 434 (1995) 3 arXiv:hep-ph/9403257; R. Adler et al. [CPLEAR collaboration], Phys. Lett. B 364 (1995) 239 arXiv:hep-ex/9511001.

[116] P. J. Steinhardt, L. Wang and I. Zlatev, Phys. Rev. D59 (1999) 123504; D. Huterer and M. S. Turner, Phys. Rev. D 60 (1999) 081301 arXiv:astro-ph/9808133; B. F. Gerke and G. Efstathiou, Mon. Not. Roy. Astron. Soc. 335 (2002) 33 arXiv:astro-ph/0201336; G. Huey and R. K. Tavakol, Phys. Rev. D 65 (2002) 043504 arXiv:astro-ph/0108517; P. S. Corasaniti and E. J. Copeland, Phys. Rev. D 65 (2002) 043004 arXiv:astro-ph/0107378; S. A. Bludman and M. Roos, Astrophys. J. 547 (2001) 77; P. H. Frampton, arXiv:astro-ph/0102344 Int. J. Mod. Phys. A 16S1C (2001) 1050 arXiv:astro-ph/0008412; S. M. Carroll, AIP Conf. Proc. 478 (1999) 291; Z. Haiman, J. J. Mohr and G. P. Holder, Astrophys. J. 553 (2000) 545 arXiv:astro-ph/0002336; P. Binetruy, Int. J. Theor. Phys. 39 (2000) 1859 arXiv:hep-ph/0005037; 
[117] P. Brax and J. Martin, Phys. Lett. B 468 (1999) 40 arXiv:astro-ph/9905040; Annalen Phys. 11 (2000) 507 arXiv:astro-ph/9912005. arXiv:astro-ph/0210533.

[118] P. Binetruy, Phys. Rev. D 60 (1999) 063502 arXiv:hep-ph/9810553; A. Masiero, M. Pietroni and F. Rosati, Phys. Rev. D 61 (2000) 023504 arXiv:hep-ph/9905346.

[119] E. J. Copeland, N. J. Nunes and F. Rosati, Phys. Rev. D 62 (2000) 123503 arXiv:hep-ph/0005222;

[120] C. Wetterich, Nucl. Phys. B302 (1988) 668; P. G. Ferreira and M. Joyce, Phys. Rev. Lett. 79 (1997) 4740; Phys. Rev. D58 (1998) 023503; E. J. Copeland, A. R. Liddle and D. Wands, Phys. Rev. D57 (1998) 4686.

[121] E. Cremmer, S. Ferrara, C. Kounnas and D. V. Nanopoulos, Phys. Lett. B 133 (1983) 61; J. R. Ellis, A. B. Lahanas, D. V. Nanopoulos and K. Tamvakis Phys. Lett. B 134 (1984) 429. J. R. Ellis, C. Kounnas and D. V. Nanopoulos, Nucl. Phys. B 241 (1984) 406. J. R. Ellis, C. Kounnas and D. V. Nanopoulos, Phys. Lett. B 143 (1984) 410. J. R. Ellis, K. Enqvist and D. V. Nanopoulos, Phys. Lett. B 147 (1984) 99; for a review see: A. B. Lahanas and D. V. Nanopoulos in [30.

[122] E. Witten, Phys. Lett. B 155 (1985) 151.

[123] P. Horava and E. Witten, Nucl. Phys. B 460 (1996) 506 arXiv:hep-th/9510209; P. Horava, Phys. Rev. D 54 (1996) 7561 arXiv:hep-th/9608019.

[124] E. Witten, arXiv:hep-ph/0002297.

[125] T. Banks, arXiv:hep-th/0007146, Int. J. Mod. Phys. A 16 (2001) 910.

[126] I. R. Klebanov and A. A. Tseytlin, Nucl. Phys. B 546 (1999) 155 arXiv:hep-th/9811035.

[127] C. Bachas, arXiv:hep-th/9503030

[128] J. M. Maldacena, Adv. Theor. Math. Phys. 2 (1998) 231 [Int. J. Theor. Phys. 38 (1999) 1113] arXiv:hep-th/9711200.

[129] For a brief, but up to date, review see: J. de Boer, Nucl. Phys. Proc. Suppl. 117 (2003) 353 arXiv:hep-th/0210224, and references therein. 